\title{
Magnetohydrodynamic models of astrophysical jets
}

\author{
IN MEMORY OF VITALY LAZAREVICH GINZBURG \\ V.S.Beskin \\ P N Lebedev Physical Institute, Russian Academy of Sciences, \\ Leninskii prosp. 53, 119991, Moscow, Russian Federation, \\ Usp. Fiz. Nauk 180, 1241-1278 (2010) [in Russian] \\ English translation: Physics - Uspekhi, 53, 1199-1233 (2010) \\ Translated by K A Postnov; edited by A Radzig
}

\begin{abstract}
In this review, analytical results obtained for a wide class of stationary axisymmetric flows in the vicinity of compact astrophysical objects are analyzed, with an emphasis on quantitative predictions for specific sources. Recent years have witnessed a great increase in understanding the formation and properties of astrophysical jets. This is due not only to new observations but also to advances in analytical theory which has produced fairly simple relations, and to what can undoubtedly be called a breakthrough in numerical simulation which has enabled confirmation of theoretical predictions. Of course, we are still very far from fully understanding the physical processes occurring in compact sources. Nevertheless, the progress made raises hopes for near-future test observations that can give insight into the physical processes occurring in active astrophysical objects.
\end{abstract}

\section{Contents}

\section{Introduction}

2 Jets

2.1 Active galactic nuclei . . . . . . . . . . . .

2.2 Microquasars . . . . . . . . . . . .

2.3 Sources of cosmological gamma-ray bursts

2.4 Radio pulsars . . . . . . . . . . . . . . .

2.5 Young stars . . . . . . . . . . . . .

3 Basics of the MHD approach

3.1 The key idea - unipolar inductor . . . 8

3.2 Grad-Shafranov equation method . . . . . 10

3.3 Supersonic flows . . . . . . . . . . 14

4 Theoretical predictions 20

4.1 Collimation . . . . . . . . . . . 20

4.2 Acceleration . . . . . . . . . . . . 25

4.3 Subsonic flows . . . . . . . . . . . 27
5 Estimation of parameters 30

5.1 Active galactic nuclei . . . . . . . . . . . 31

5.2 Microquasars . . . . . . . . . . . . . . 33

5.3 Sources of cosmological gamma-ray bursts 33

5.4 Radio pulsars . . . . . . . . . . . . . . . 34

5.5 Young stars . . . . . . . . . . . . 34

6 Conclusions 35

7 Appendix 35

\section{Introduction}

This review was not specially written for the Uspekhi Fizicheskikh Nauk (Physics-Uspekhi) issue devoted to the memory of V L Ginzburg. Nevertheless, I would like to hope that the spirit of this review is close to that of other papers in this issue. I was happy to work closely with Vitaly Lazarevich for more than 30 years, starting from my graduate student days, and he definitely played a significant role in my scientific development. So, this review, hopefully, bears a fraction of the soul of Vitaly 1 Lazarevich.

Vitaly Lazarevich was a passionate person. Astrophysics, undoubtedly, fascinated him most. But the scale of his personality was such that this passion did not separate, but instead united, people. So, it is not surprising that at the Lebedev Institute the astrophysical seminar headed by Vitaly Lazarevich for more than several decades continues, and scientists from many institutes participate in its work. The Department of Physics and Astrophysics Problems at Moscow Institute of Physics and Technology (MIPT), which Vitaly Lazarevich founded in 1968 and headed until recently, continues to be one of the leading institutions in teaching young astrophysicists. Numerous pupils of V L Ginzburg and pupils of his pupils working in the leading astrophysical centers of the world keep his unique trademark in their studies.

The astrophysical heritage of V L Ginzburg is enormous. He obtained fundamental results in the theory of propagation of electromagnetic waves in cosmic plasma, 
in the theory of the origin of cosmic rays, and in the theory of neutron stars and black holes. In all cases, a simple model allowing the understanding of the essence of physical process in observed astrophysical sources laid the basis of the theory. The present review, hopefully, was written in the same spirit.

Astronomy, as follows from the very appellation, is the science that stemmed from the observations of stars. During hundreds of years the people observed stars in the sky and gained insight into the laws of Nature. The stars appeared to be always unchanged and existing for an infinite amount of time. After the appearance of spectral analysis, the first astrophysical observations and, later, the theory of radiation generally confirmed this point of view. The lifetimes of most stars turned out to be comparable to the age of the Universe. Thus, in the 1950s, when radio astronomy began, stars emitting thermal radiation seemed to be the main objects for studies. In radio astronomy, the brightness temperature remains even now the basic characteristic of radiation intensity.

However, the first radio astronomical observations, and especially observations in the X-ray and gamma-ray ranges, which started in the middle of the $1970 \mathrm{~s}$, discovered numerous nonthermal sources in the Universe. These objects are sufficiently compact (i.e., the spatial resolution of the existing detectors is insufficient to determine their internal structure) and, in addition, are highly variable. In active galactic nuclei the variability timescale (months or sometimes even days) is small according to the cosmic timescale, with the variability timescale of radio pulsars and sources of gamma-ray bursts being the fractions of a second, which is small even to Earth's measures. The activity, i.e., high variability on timescales $\tau \sim R / c$, as well as the generation of nonthermal radiation indicates that in most cases we are dealing with relativistic objects, namely, with objects in which matter moves with velocities close to the speed of light.

Jet eruptions represent one of the visible appearances of the activity of compact astrophysical objects. We shall briefly discuss their properties in Section 2. They are observed in both relativistic objects (such as active galactic nuclei and microquasars) and in young stars where the motion of matter is definitely nonrelativistic. This means that we are dealing with some universal and extremely efficient mechanism of energy release. Therefore, the key theoretical problems include the question of the energy source of the activity of compact objects, the understanding of their energy release mechanism, and the collimation of matter outflows. We shall postpone the detailed discussion of arguments against alternative models until the next section, and here we only remind the main arguments favoring the magnetohydrody-namic model of activity of compact sources, which is accepted by most astrophysicists.

The model of the unipolar inductor, i.e., the source of direct current, lies at the heart of the magnetohydrodynamic approach. As we shall show in Section 2, conditions for the existence of such a 'central engine' are satisfied in all the compact sources discussed below.
Indeed, all compact sources are assumed to harbor a rapidly spinning central body (black hole, neutron star, or young star) and some regular magnetic field, which leads to the emergence of strong induction electric fields. The electric fields, in turn, lead to the appearance of longitudinal electric currents and effective particle acceleration. The collimation mechanism in this model is related to the well-known property of mutual attraction of parallel currents.

The first studies of the electromagnetic model of compact sources (namely, radio pulsars) were carried out as early as the end of the 1960s [1-4]. It was evidenced that there are objects in the Universe in which electrodynamical processes can play the decisive role in the energy release. Then, in $1976 \mathrm{R}$ Blandford [5] and R Lovelace [6] independently suggested that the same mechanism can also operate in active galactic nuclei. In the same year, G S Bisnovatyi-Kogan, Yu P Popov, and A A Samokhin proposed a magnetorotational mechanism of the supernova explosion [7] (i.e., the model of an essentially nonstationary phenomenon), in which jet eruptions can also be formed [8]. This model has remained the leading one for nearly 40 years. However, only recently have some key properties become clear. This is related both to advances in the theory which have at last formulated sufficiently simple analytical relations, and to the breakthrough in numerical simulations which confirmed theoretical predictions.

The reader can find the detailed introduction to the analytical theory in the author's monograph [9] (see also the review in Physics-Uspekhi in 1997 [10]). However, first, the monograph was devoted to the basics of the theory, and qualitative predictions for specific astrophysical sources were discussed only very briefly. Second, the monograph clearly could not include the results of numerical calculations carried out in the last five years since its publication. This is the main reason for writing the present review. In addition, here we shall correct formulas from the monograph in which misprints were found.

Of course, we are still far away from the full understanding of the essence of physical processes proceeding in compact sources. In fact, now we have only agreement between theory and numerical modeling. All results have been obtained applying ideal one-liquid magnetohydrodynamics, though by different methods (the theory is based on stationary equations, while numerically the time relaxation problem is solved). In particular, it is not yet clear which of the main physical characteristics of the central engine (such as the mass of the central body or its rotation velocity) should fully determine the observed energy release. Nevertheless, the progress achieved over recent years raises hopes for test observations already in the nearest future, which can give insight into physical processes occurring in active astrophysical sources. 


\section{Jets}

\subsection{Active galactic nuclei}

The main properties of the central engine in active galactic nuclei, which are presently accepted by most astrophysicists, can be summarized as follows $[11,12]$. In the center of the host galaxy there is a supermassive black hole (its mass reaches $10^{6}-10^{9} M_{\odot}$, where $M_{\odot} \approx 2 \times 10^{33} \mathrm{~g}$ is the mass of the Sun), onto which accretion of the surrounding matter occurs [13]. Only in this case it is possible to explain the very high efficiency of the energy release and the compactness of the central engine. The energy source of activity of galactic nuclei can be related to both the rotational energy of the black hole, viz.

$$
E_{\mathrm{tot}}=\frac{J_{\mathrm{r}} \Omega_{\mathrm{H}}^{2}}{2} \approx 10^{62}\left(\frac{M}{10^{9} M_{\odot}}\right)\left(\frac{\Omega_{\mathrm{H}} r_{\mathrm{g}}}{c}\right)^{2} \mathrm{erg},
$$

and the energy of the accreting matter. Here $r_{\mathrm{g}}=2 G M / c^{2}$ is the radius of the black hole, $J_{\mathrm{r}}$ is the moment of inertia, $\Omega_{\mathrm{H}}$ and $M$ are the angular velocity and the mass of the black hole, respectively, and $c$ is the speed of light. The existence of supermassive objects is also supported by the fact that the Eddington luminosity

$$
L_{\mathrm{Edd}} \approx 10^{47}\left(\frac{M}{10^{9} M_{\odot}}\right) \operatorname{erg~s}^{-1}
$$

(i.e., the luminosity at which the gravitational force acting on the accreting matter is balanced by the radiation pressure force) is close to the characteristic luminosity of active galactic nuclei [14]. Moreover, the duration of the active phase $\tau_{\mathrm{D}}=E_{\mathrm{tot}} / L_{\mathrm{Edd}}$ estimated using formulas (1) and (2) is on the order of $10^{7}$ years, which is also in agreement with observations.

Further, it is usually assumed that the accretion of matter proceeds through a disc [15]. Thus, the preferential direction - the axis of rotation - emerges naturally in space, along which the formation of jets is possible. As a black hole itself cannot have the self-magnetic field (the so-called 'no-hair theorem'), the generation of a large-scale magnetic field in the vicinity of the black hole is believed to occur in the accretion disc [16-18].

According to the modern concept, massive central objects are present in most galaxies and remain active only if a sufficient amount of matter falls on them. This restricts their active lifetime. Unfortunately, as stated above, the angular resolution of modern detectors does not allow us to directly observe plasma flow on the scales comparable to the black hole size $r_{\mathrm{g}} \approx 3 \times 10^{14}\left(M / 10^{9} M_{\odot}\right) \mathrm{cm}$. Therefore, we have to judge the activity of galactic nuclei only using indirect evidence, by observing flows on much larger scales.

Let us remember that the diffuse radio emission around active galaxies is observed from regions located at distances of tens or even a hundred kiloparsecs from their nuclei. Very shortly after the discovery of these

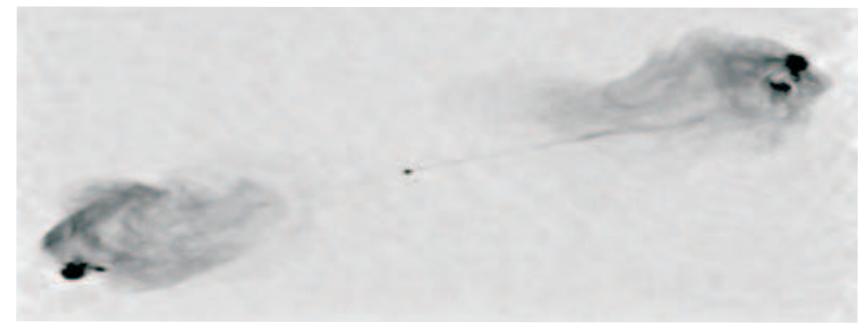

Figure 1: Radio image $(5 \mathrm{GHz})$ of active regions and jet eruptions from the nucleus of the Cygnus A galaxy [20]. The distance between bright spots is about $80 \mathrm{kpc}$, which is 9-10 orders of magnitude greater than the size of the central black hole.

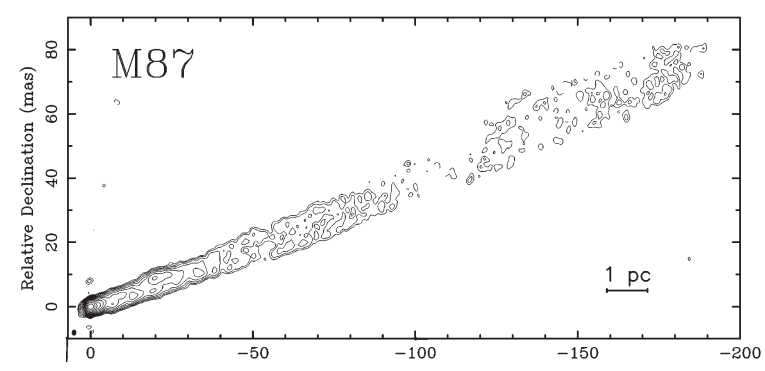

Figure 2: Radio image of the jet eruption from the galaxy M87 near the central engine [22]. The jet transverse size is about $1 \mathrm{pc}$.

regions at the beginning of the $1960 \mathrm{~s}$, this emission was associated with collimated plasma ejections (jets) flowing out the galactic nuclei [12]. It is precisely these jets that transport matter and energy from the active nuclei to those regions (Fig. 1). Observations show that the jets can be accelerated and collimated very close to a galactic nucleus. For example, in the case of the nearest active galaxy M87 the formation of the jet occurs within a radius of $60 r_{\mathrm{g}}$ from the nucleus [19]. In recent years, the internal structure close to the jet base was resolved in several sources, where the jet tranverse dimension usually does not exceed several parsecs $[21,22]$ (Fig. 2).

The matter in jets from active galactic nuclei has a very high energy - the bulk Lorentz factor of a jet is at least a few unities. For example, this motion is directly observed in the M87 galaxy, with the bulk Lorentz factor of the outflow being $\gamma \approx 6$ [23]. In many cases, the matter continues moving with relativistic velocities up to huge distances from the nucleus before noticeable braking due to interaction with the ambient intergalactic medium. Another peculiar feature of jets is their high degree of collimation within a cone characterized by an opening angle of only several degrees.

Unfortunately, observations do not yet allow reliable estimations of the energy and mass fluxes in jets from 
active galactic nuclei, of the magnitude of the magnetic field both close to the black hole and in the jet itself, or of the composition of jet eruptions. The spectrum of radiation from galactic jets (in contrast, for example, to the spectrum of jets from young stars) does not exhibit any spectral features of moving matter, i.e., neither atomic (ionic) lines nor the electron-positron pair annihilation line are observed. To this regard, there are arguments both in favor [24] and against [25] the leading role of electron-positron plasma, so it is now impossible to say exactly which mechanism of energy transfer to the jet actually operates.

Where a physical nature of the galactic nucleous activity is concerned, several mechanisms of particle acceleration and jet collimation have been proposed, but so far there is no definite answer as to which of them are actually realized. It is possible that different mechanisms operate in different sources, or, just the opposite, all mechanisms are realized simultaneously.

Gas-dynamic acceleration. The acceleration and collimation of a jet can be related to the presence of an ambient medium with high pressure which decreases with distance from the center $[26,27]$. Such a medium could play the role of an external wall collimating the outflow. The pressure of the external hot medium can, in principle, be estimated from X-ray observations [28]. This mechanism possibly explains how weak jets in our Galaxy and in some Seyfert galaxies (i.e., low-active galaxies) are formed. On the other hand, the observed pressure of the hot matter around the most powerful jets from active galactic nuclei is not sufficiently high, and there must be an alternative mechanism of plasma confinement.

Acceleration by radiation. As the photon density near the central source can be very high, the radiation-driven mechanism of jet matter acceleration by radiation pressure was proposed $[29,30]$. In this model, it is assumed that the inner parts of the disc can serve as a nozzle directing matter outflows accelerated by the radiation pressure. However, this mechanism also meets some difficulties. For example, there is no correlation between the jet power and the luminosity of the source - many sources with very powerful jets are low-luminous sources [31]. Another difficulty comes from the fact that, starting from the sufficiently low particle energies $\gamma \approx 3$, the radiation field more effectively brakes particles than accelerates them [32]. This contradicts observations of 'superluminal' sources in which the energy of plasma particles is much higher. In addition, if the jet was formed in a system with a thin accretion disc emitting radiation more or less isotropically, additional mechanisms for the jet collimation should be invoked. A modification of this model involving the formation of a funnel in a thick accretion disc can explain the initial jet collimation, but there are indications that such a structure is unstable [31].

Magnetohydrodynamic mechanism. As noted above, most researchers favor the magnetohydrodynamic model of jet formation. The magnetohydrodynamic (MHD) model was successfully utilized to describe many processes in active nuclei, and, in particular, in connection with the problem of the origin and stability of jets, as well as to explain the energetics of processes proceeding near the central black hole. The magnetic field here is the natural link between the central engine and the jet. Moreover, in this model it is easy to understand why the jet matter can predominantly consist of electron-positron plasma. As was shown in Refs [33-35], it can be generated on the magnetic field lines threading the black hole horizon.

In the simplest version, the picture is as follows: the regular magnetic field generated in the disc links the spinning central engine (the disc and the black hole) with infinity. The plasma outflow occurs along the magnetic field lines; the electromagnetic energy flux is also directed along the magnetic field lines. The longitudinal electric current flowing along the jet forms a toroidal magnetic field, and the magnetic field pressure associated with this toroidal component can collimate the jet.

It should be noted, however, that in a real astrophysical system the total current flowing from the central engine should vanish, so the Ampere force in the current closure region will, on the contrary, decollimate the flow (the antiparallel currents repulse). Therefore, an external medium (for example, a subrelativistic wind outflow from the accretion disc) is necessary to collimate the jet. In addition, the question as to whether it is possible to consider a black hole immersed in the external magnetic field as a unipolar inductor turned out to be also rather nontrivial. It required almost 30 years of studies after the paper by Blandford and Znajek [33], which laid the basis of the theory in 1977, before the needed clarity was reached in this question. We shall discuss these points in more detail in Section 3.

\subsection{Microquasars}

Microquasars comprise galactic objects in which the jet formation is due to accretion onto a compact relativistic object (neutron star or black hole). In other words, all microquasars reside in sufficiently close binary systems in which the effective flow of matter from the star companion occurs. The rate of matter inflow in such systems is larger than can be swallowed by the central object. As a result, some accreting matter that carries, in particular, an excessive angular momentum is expelled from the system in the form of jets. Observations of microquasars show that jets are related to thick accretion discs. In other words, no jets are known for systems with thin discs. The reason for that is unclear: either a thin disc insufficiently collimates the outflow, or the magnetic field generated by the thin disc is not strong enough.

Microquasars represent a small population of objects, including only around ten sources [36], with only half of them demonstrating noticeable relativistic jets $(v>0.9 c)$. The characteristic longitudinal size of jets is usually $0.1 \mathrm{pc}$, with the jet spread angle being within several degrees (Fig. 3). The total energetics are about $10^{37} \mathrm{erg} \mathrm{s}^{-1}$. Due to the relativistic velocity of the bulk motion of matter in the jets, some sources demonstrate the superluminal motion effect, with the apparent an- 


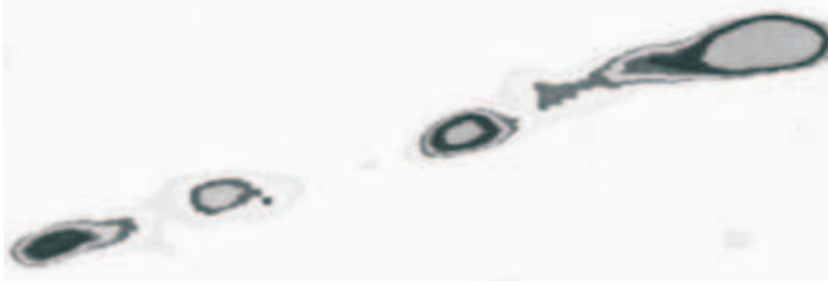

Figure 3: Radio image of the jet from the microquasar iE1740.7-2942. The characteristic length of the jet measures $0.1 \mathrm{pc}[38]$.

gular velocity being several orders of magnitude larger (due to a relative proximity of these objects) than that observed in jets from active galactic nuclei.

Historically, the first revealed object of this class was the famous source SS433 [37] in which, however, the gas ejection velocity in jets is only $0.26 c$. Such a velocity can be easily explained by the radiation pressure from highly heated internal regions of the accretion disc. As for relativistic jets, the first source was discovered only in 1994 [38]. Since the appearance of near-light velocities due to radiation or gas pressure is problematic, it has not been ruled out that, to explain them, an electrodynamic model similar to that used in explaining the origin and collimation of extragalactic jets should be invoked again. This model is also supported by the fact that in all but one microquasar (SS433) no emission lines from jets are observed. This indirectly points to the electron-positron composition of matter in jets [36]. Finally, it should be noted that in most microquasars the jet is separated in individual blobs at large distances from the central engine, which is thought to be due to a long duty cycle of the work of the central engine.

\subsection{Sources of cosmological gamma-ray bursts}

As regards the sources of cosmological gamma-ray bursts, there are indirect, although sufficiently reliable, arguments in favor of the presence of jets related exactly to relativistic strongly magnetized outflows, which we shall discuss in this review. It is well known that the discovery of the optical afterglow [39], as well as afterglows in other spectral ranges, which allowed the measurement of the distance to these sources from the observed redshifts of the host galaxies, put serious constraints on their energetics [40]. If the observed gamma-ray radiation were emitted isotropically, the total energy release for the typical distance to these sources of several gigaparsecs would reach $10^{54} \mathrm{erg}$. However, we do not know at present processes with such huge energy liberation. On the other hand, the small duration of the burst $(\sim 10 \mathrm{~s})$ restricts the size of the emission region, which, in turn, does not allow us to explain the observed nonthermal gamma-ray spectra, since the optical depth in the source proves to be very high [41].

If it is assumed that gamma-rays are emitted within a narrow cone angle $\vartheta \sim 1^{\circ}$, the observed energy can be reduced to $10^{51} \mathrm{erg}$, which is already to an order of magnitude of the energy release during supernova explosions. On the other hand, the observed optically thin nonthermal gamma-ray spectra immediately imply the presence of ultrarelativistic outflows with bulk Lorentz factors of $\sim 100-300$. Only in this case can the compactness problem of the source be resolved, since the estimated size of the emitting region also increases as the square of the bulk Lorentz factor (i.e., by $10^{4}-10^{5}$ times), and the optical depth, which is proportional to the density multiplied by the size of the region, decreases respectively by $10^{8}-10^{10}$ times.

However, the ultrarelativistic character of the outflow, in turn, puts constraints on the particle composition in the jet, since the presence of a significant fraction of baryons with such energy in the outflow would contradict the total energy release in the gamma-ray burst. Therefore, the contribution of protons must be smaller than $10^{-2}$ of the total number of particles, so that only electron-positron jets should be considered. The existence of jets is also evidenced by the presence of the characteristic bend of the light curve of the afterglow, when the power law index $\alpha$ in the radiation intensity dependence on time, $W_{\text {tot }} \propto t^{-\alpha}$, changes from $\alpha \approx 1.1$ to $\alpha \approx 2.0$ after a span of about a few days following the burst. This effect is related to the cessation of relativistic contraction of the radiation cone in the direction of particles' motion toward an observer. Incidentally, this model allowed independent confirmation of the jet spread angle $\vartheta \sim 1^{\circ}$ and the bulk Lorentz factor $\gamma \sim 100-300$ [42].

The nature of the central engine giving rise to strongly magnetized jets can be usually related to the collision of two neutron stars $[43,44]$ or of a neutron star and a black hole [45], or, most likely, to the collapse of the massive core of an unusual supernova [46, 47]. However, in most models a rapidly spinning solar-mass black hole ultimately emerges, which loses its rotation energy via the Blandford-Znajek process [45, 48 50]. Indeed, as we have seen, this process easily provides a natural explanation for both the low baryonic load of the jet and the large bulk Lorentz factors of jet particles. In other words, the model again is constructed similarly to the scheme proposed for active galactic nuclei. In particular, the key processes here also include the magnetic field generation in the plasma around the black hole, the interaction of the black hole with the accretion disc via magnetic field lines, and the generation of particles in the magnetosphere. To explain the observed energy release, it is necessary to assume that the magnetic field near the black hole must be as high as $10^{14}$ or even $10^{15} \mathrm{G}$. The generation of such a high field is thought to be possible in nonstationary processes like the supernova core collapse or binary neutron star coalescence [51, 52]. 


\subsection{Radio pulsars}

The discovery of radio pulsars at the end of the $1960 \mathrm{~s}$, which are the sources of pulsating cosmic radio emission with the characteristic period $P \sim 1 \mathrm{~s}$ [53], is definitely one of the major astrophysical discoveries of the 20th century. Indeed, for the first time a new class of cosmic sources related to neutron stars, whose existence was theoretically predicted away back in the 1930s [54], was discovered. Neutron stars (mass of about $1.2-1.4 M_{\odot}$, and radius $R$ of only $\left.10-15 \mathrm{~km}\right)$ must result from the catastrophic compression (collapse) of usual massive stars at the late stage of their evolution or, for example, of white dwarfs whose mass exceeds the Chandrasekhar mass limit of $1.4 M_{\odot}$ due to accretion from the companion star. It is this formation mechanism that provides the simplest explanation for both small spin periods $P$ (the smallest known spin period $P=1.39 \mathrm{~ms}$ ) and superstrong magnetic fields with $B_{0} \sim 10^{12} \mathrm{G}[1,2]$.

Interestingly, the basic physical processes determining the observed activity of radio pulsars were understood almost immediately after their discovery. For example, it became clear that highly regular pulsations of observed radio emission are related to the rotation of neutron stars. Next, radio pulsars are powered by the rotational energy of the neutron star, and the mechanism of energy release is related to their superstrong magnetic field with $B_{0} \sim 10^{12} \mathrm{G}$. Indeed, energy losses estimated using the simple magnetodipole formula [44] are as follows:

$$
W_{\mathrm{tot}}=-J_{\mathrm{r}} \Omega \dot{\Omega} \approx \frac{1}{6} \frac{B_{0}^{2} \Omega^{4} R^{6}}{c^{3}} \sin ^{2} \chi
$$

where $J_{\mathrm{r}} \sim M R^{2}$ is the moment of inertia of the neutron star, $\chi$ is the angle between the magnetic dipole axis and the spin axis, and $\Omega=2 \pi / P$ is the angular velocity of the neutron star rotation. For most pulsars, energy losses range from $10^{31}-10^{34} \mathrm{erg} \mathrm{s}^{-1}$. These energy losses exactly correspond to the observed spin-down rate $\mathrm{d} P / \mathrm{d} t \sim 10^{-15}$, or to the spin-down time $\tau_{\mathrm{D}}=P / \dot{P} \sim$ 1-10 mln years. Let us keep in mind that the fraction of radio emission amounts to only $10^{-4}-10^{-6}$ of total energy losses. For most pulsars this corresponds to $10^{26}-10^{28} \mathrm{erg} \mathrm{s}^{-1}$, which is $5-7$ orders of magnitude less than the luminosity of the Sun.

As shown in Refs [56, 57], the actual energy losses cannot be due to magnetodipole radiation because the plasma that fills the magnetosphere will fully screen the low-frequency radiation from the neutron star. However, energy losses can be caused by longitudinal electric currents circulating in the magnetosphere and looped across the surface of the central engine. As a result, in this case, too, the main energy release near the neutron star is related to the electromagnetic energy flux (the Poynting vector flux), and the total energy losses can be again estimated using formula (3).

Most radio pulsars constitute single neutron stars. Of the 1880 pulsars known by the middle of 2010 , only 140 were members of binary systems. However, in all these cases it is reliably known that there is no somewhat appreciable mass transfer from the companion star to the neutron star. Since, as already stressed, the radio luminosities of pulsars are low, the modern sensitivity of detectors allows observations of pulsars only up to distances of 3-5 kpc, which is smaller than the distance to the galactic center. Therefore, we can observe only a small fraction of all 'active' pulsars. The total number of neutron stars in our Galaxy must be around $10^{8}-10^{9}$ Such a big number of extinguished neutron stars can be naturally related to the small duration of their active life, as discussed above.

The jets are only observed in Crab and Vela radio pulsars [58, 59], which is not surprising, since, in contrast to the compact objects considered above, the pulsar magnetosphere is not axisymmetric. On the other hand, only axisymmetric configurations were actually considered until recently in the theory of pulsar wind. Based on these studies, the main features of strongly magnetized winds were understood. Nevertheless, even in this approximation for smooth flows, it has thus far been impossible to construct a self-consistent model which jointly describes the energy transfer from the neutron star surface to infinity and includes effective particle acceleration, i.e., an almost complete transformation of the electromagnetic field energy into the energy of the plasma flowing out. Because of this, different models are actively being discussed at present, which, to various degrees, propose going beyond the framework of the 'classical' scheme (see, for example, Refs [60-62]).

Indeed, observations show that most energy far from the neutron star must be carried by relativistic particles. For example, the analysis of the emission from the Crab Nebula in the shock region located at a distance of $\sim 10^{17} \mathrm{~cm}$ from the pulsar in the region of interaction of the pulsar wind with the supernova remnant definitely shows that the total flux $W_{\text {em }}$ of the electromagnetic energy in this region is no more than $\sim 10^{-3}$ of the particle energy flux $W_{\text {part }}[63]$. Thus, the Poynting vector flux in the asymptotically remote region must be completely converted into the outgoing plasma flux. The presently known axisymmetric numerical models of jets from radio pulsars [64-66] were constructed exactly under this assumption.

However, the transformation apparently occurs already much closer to the neutron star, namely at distances comparable to the size of the light cylinder. This is evidenced by the detection of variable optical emission from companions in some close binary systems involving radio pulsars [67]. This variable optical emission with a period equal exactly to the orbital period of the binary can be naturally related to the heating of the companion's part facing the radio pulsar. It was found that the energy reradiated by the companion star almost matches the total energy emitted by the radio pulsar into the corresponding solid angle. Clearly, this fact cannot be understood either in the magnetodipole radiation model or by assuming a Poynting-dominated strongly magnetized outflow, since the transformation coefficient of a low-frequency electromagnetic wave cannot be close to unity. Only if a significant fraction of the energy is related to the relativistic particle flux can the heating of 


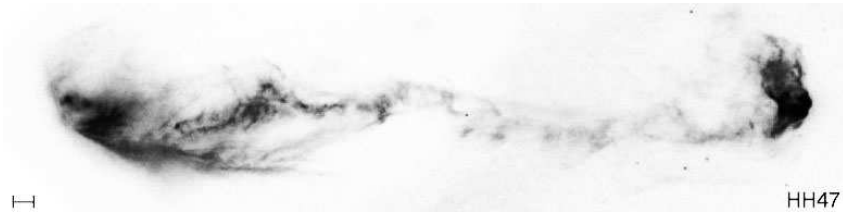

Figure 4: Optical image of jets from the system HH47 (see, for example, Ref. [70]). The scale corresponds to 1000 a.u.

the star's surface be effective enough. Therefore, the socalled $\sigma$-problem - the question as to how the energy is transferred from the electromagnetic field to particles in the pulsar wind - remains one of big puzzles in modern astrophysics.

\subsection{Young stars}

Jets from young stars were indirectly discovered at the beginning of the 1950s, when G Herbig and G Haro [68, 69] discovered a new class of extended diffuse objects usually existing in pairs and, as became clear later, connected by thin jets with young rapidly rotating stars [70]. The formation of such jets can naturally be related to the need of removing most effectively the excessive angular momentum that prevents the formation of a star. As we see, the situation here is quite similar to that with active galactic nuclei, where first a diversity of different types of sources (quasars, Seyfert galaxies, and radio galaxies) were discovered, and only later on did it become clear that the activity of all these sources has a similar nature. Moreover, the similarity of the observational features suggests that the physical mechanism of jet formation from young stars can also be similar to that from active galactic nuclei. And this is despite the fact that physical conditions near a young star (mass of order 3-10 $M_{\odot}$, and total energy release ranging from $10^{31}$ to $10^{36} \mathrm{erg} \mathrm{s}^{-1}$ are dramatically different from those in the centers of active galactic nuclei. One of the main differences here is the nonrelativistic character of gas outflow from young stars.

Presently, more than 250 Herbig-Haro objects are known [71]. As shown in Fig. 4, they represent bright condensations with an angular size of several seconds of arc (linear size of order 500-1000 a.u.), usually surrounded by a bright diffuse envelope. Their spectra mainly show emission lines of hydrogen and some other low-excitation elements. A shock wave propagating with velocities $40-200 \mathrm{~km} \mathrm{~s}^{-1}$ through a gas with a density of $\sim 10^{2} \mathrm{~cm}^{-3}$ is apparently the main source of excitation [70].

As in the case of radio galaxies, the activity of HerbigHaro objects is dictated by collimated outflows which are well seen in forbidden lines. Nearly $60 \%$ of the objects demonstrate both jets, while in other cases the receding jet is blocked by the accretion disc. The extent of the optical jets is of order $0.01-2 \mathrm{pc}$, and their velocity reaches

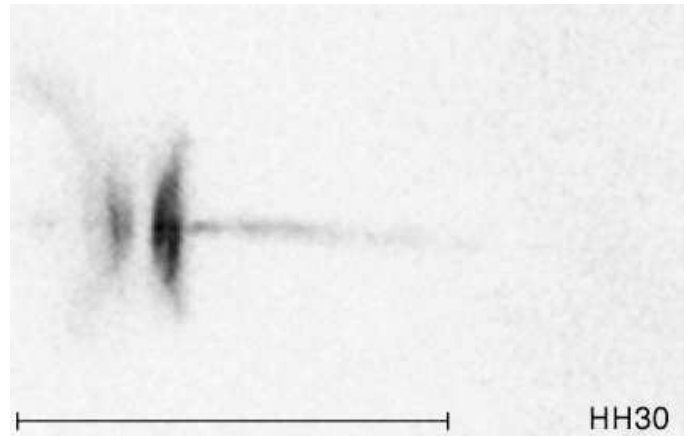

Figure 5: Formation of a jet from a young star in the system HH 30 [70]. The accretion disc is clearly seen. Here also the scale corresponds to 1000 a.u.

$600 \mathrm{~km} \mathrm{~s}^{-1}$. The gas density in the jets is estimated to be $10-100 \mathrm{~cm}^{-3}$, and the mass outflow rate comes to $10^{-9}-10^{-10} M_{\odot} \mathrm{yr}^{-1}$. The degree of collimation of the jets (the ratio of the observed length to the width) can be as high as 30 . The total jet opening angle is in the range of $5-10^{\circ}$. In addition to highly elongated jets, molecular outflows with a much smaller collimation degree are observed near young stars. Their size may run to 0.04-4 pc, and the velocity of gas motion does not exceed $5-100 \mathrm{~km} \mathrm{~s}^{-1}$. Here we should stress that this velocity is much higher than the speed of sound in an outflow with a temperature of only $10-90 \mathrm{~K}$. The total mass of the ejected gas is estimated to be $0.1-200 M_{\odot}$, and the total kinetic energy stored in the molecular outflows can reach $10^{43}$ and even $10^{47} \mathrm{erg}$. The direct observation of rotation of the jets is the most important recent discovery. The characteristic velocities at an axial distance of $20-30$ a.u. range from $3-10 \mathrm{~km} \mathrm{~s}^{-1}[72,73]$. There is also direct evidence of the spiral structure of the magnetic field in the jets [74]. All these facts unambiguously support the MHD model.

As in jets from microquasars, a strong instability frequently develops in collimated outflows from young stars at large distances from the central engine (see Fig. 4), so that the outflow is split into separate blobs. On the other hand, as seen from Fig. 5, the flow near the base of the jet can be considered sufficiently regular.

As for the physical nature of collimated jet formation, this question is still far from solved. It is only clear that the power of the central engine is always sufficient to accelerate the outflowing gas; however, the mechanism of energy transformation remains unclear. We stress that in contrast to relativistic galactic objects (for example, microquasars), where the formation of jets is possibly caused by supercritical accretion, the luminosity in young stars never approaches the Eddington limit. On the other hand, it is clear that the key role in the collimated outflow formation is just played by accretion discs which undoubtedly exist around young stars. This is supported by the direct correlation between the power of the gas flux and the mass of the disc, estimated from 
its luminosity, as well as some other correlations [ $[75,76]$. The parameters of the discs can be very different. For example, their masses range from $0.1-100 M_{\odot}$, while the outer radii can vary from 10 a.u. to $0.1 \mathrm{pc}$.

It is important that, in contrast to discs around relativistic objects (neutron stars and black holes), the gas temperature in discs around young stars is only 20-100 K. As a result, as in the case with active galactic nuclei, neither the radiation pressure force nor gas pressure can explain the high velocities observed in the collimated outflows [71]. Therefore, to explain the jet formation and particle acceleration, models in which the magnetic field plays the key role and effectively mediates the interaction between the accretion disc and the jet were invoked once again. Because the real structure of the magnetic field in the proximity of a young star is presently unknown, here, too, both models in which the magnetic field of the star itself has a dominant role [77] and models in which the magnetic field of the disc plays the decisive role $[75,78]$ have been proposed. It is seen that here we meet the same problems regarding the structure of the initial magnetic field as in the study of the black hole magnetosphere.

\section{Basics of the MHD approach}

\subsection{The key idea - unipolar inductor}

As already said, the notion of a unipolar inductor is the main physical idea that underlies the MHD theory of compact objects. Referring to Fig. 6, a rotating magnetized ball can serve as the battery that determines the energy release from the central engine. Indeed, assuming the high conductivity of the ball, the freezing-in condition of the magnetic field, viz.

$$
\mathbf{E}_{\text {in }}+\frac{\boldsymbol{\Omega} \times \mathbf{r}}{c} \times \mathbf{B}_{\text {in }}=0
$$

(i.e., simply the condition that the electric field in the rotating reference frame vanishes), leads to the appearance of the potential difference $\delta U$ between points $a$ and $b$. To an order of magnitude, this potential difference can be

$$
\delta U \sim E R_{0} \sim \frac{\Omega R_{0}^{2}}{c} B,
$$

where $R_{0}$ is the transverse size of the working area. As a result, the total energy release $W_{\text {tot }}$ on the external load $\mathcal{R}$ will be given as

$$
W_{\text {tot }}=I \delta U,
$$

where the electric current $I=\delta U / \mathcal{R}$. Here, however, several conditions should be met. First, the electric circuit must touch the ball at different latitudes, i.e., at points with different electric potentials. Second, the electric circuit should rotate with an angular velocity $\Omega$ different from that of the magnetized ball. The current flowing along a wire tightly welded on the ball will be absent.

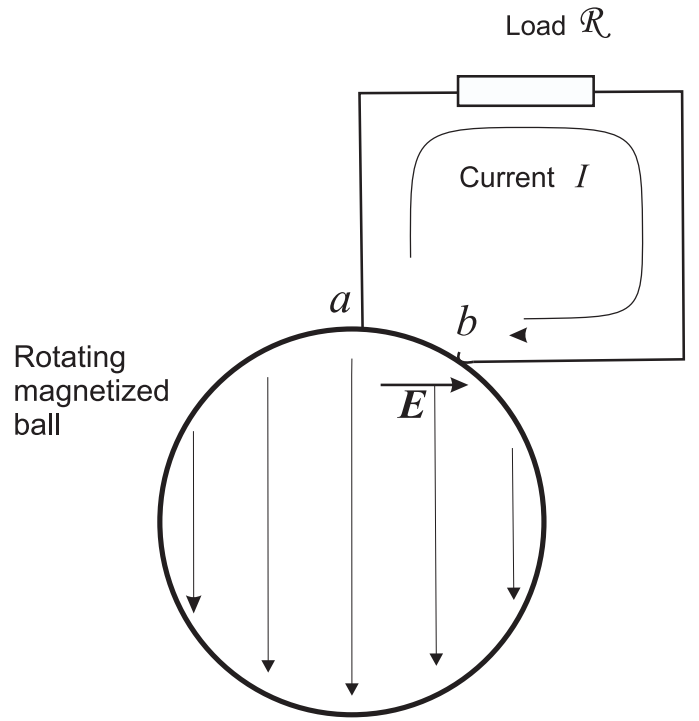

Figure 6: The unipolar inductor as the source of a direct current. Inside the magnetized ball, the electric current flows against the electric field direction.

We stress that the energy source [electromotive force $(\mathrm{EMF})]$ in the unipolar inductor is due to the kinetic energy of rotation. Indeed, as seen from Fig. 6 , charges inside the ball move against the direction of the electric field. This becomes possible due to the force by which the lattice acts on charges carried along the wire, which violate the freezing-in condition inside the ball. Conversely, the Ampere force acting from the side of the surface electric current on the ball's material brakes its rotation. Therefore, the principle of work of the unipolar inductor (or, as it is sometimes called, the unipolar Faraday generator) is not the Faraday effect as such (where the EMF induced in a current loop depends on the variation of the magnetic flux), since the flux through the circuit remains constant. Notice that the reverse situation is also possible: if one applies a potential difference to a magnetized ball (i.e., if one replaces the load in Fig. 6 by the voltage source), the ball starts rotating. On the site http://fiziks.org.ua/samyj-prostoj-v-mire-elektrodvigatel/ which is devoted to laboratory studies in secondary school, one can find a video illustrating the work of such a device.

As we have understood, for the central engine to operate it is necessary to have:

- rotating body;

- regular magnetic field, and

- well-conducting wire.

Then the current, and hence the energy losses, will be determined by the value of the external resistance $\mathcal{R}$. 
Let us see now whether these conditions can be met in compact astrophysical objects.

As we have seen, a central rotating body in active astrophysical sources is undoubtedly present. For example, the spin periods of young stars are about several days (the inner parts of accretion discs rotate even faster). The spin periods of most radio pulsars are close to $1 \mathrm{~s}$; however, they can be as small as a few milliseconds, which is already close to the limiting speed of rotation $(\Omega R / c \sim 0.1)$. The rotational velocities of black holes in active galactic nuclei, to tell the truth, are unknown, but we can suppose that due to disc accretion (it is in this way that the millisecond-period pulsars are thought to have been spun up) their spin parameter $\Omega_{\mathrm{H}} R / c=a / 2 M$ (see the Appendix) can also be sufficiently large. For example, the estimate of the black hole rotational velocity in the nucleus of Seyfert galaxy MCG 06-30-15, as inferred from the iron 6.4-keV line profile distortion, yields $a / M=0.989_{-0.002}^{+0.009}$ [79] (see also Ref [80]). As a result, the kinetic energy of rotation $\mathcal{E}_{\text {kin }}=J_{r} \Omega^{2} / 2$ stored in the central engine turns out to be quite sufficient to explain the energy source of activity of compact objects.

There are no particular problems with a regular magnetic field, either. In young stars, the proper magnetic field $B_{0}$ is measured directly and can be as high as $10^{3} \mathrm{G}$ [71]. At present, there are no direct observations of magnetic fields in radio pulsars, but they can be measured in X-ray (accreting) pulsars, which are also neutron stars [11]. Therefore, nobody now doubts that the magnetic field of a neutron star can reach $10^{12} \mathrm{G}$, and even extend up to $10^{15} \mathrm{G}$ in magnetars [81]. The situation is worse with the magnetic fields of supermassive black holes. As is well known, a black hole cannot have a proper magnetic field, but the field can be generated in the surrounding accretion discs [82]. Unfortunately, so far there is no self-consistent theory of such generation, so we have to apply the estimate $B_{0} \sim B_{\mathrm{Edd}}$, where

$$
B_{\mathrm{Edd}} \approx 10^{4}\left(\frac{M}{10^{9} M_{\odot}}\right)^{-1 / 2} \mathrm{G} \text {. }
$$

Let us keep in mind that such an estimate comes from the simple assumption that the energy density of the magnetic field is comparable to the total energy density in the accreting plasma yielding the Eddington luminosity (2). Clearly, estimate (7) represents rather an upper limit of the magnetic field near the black hole. In particular, it does not take into account the contribution from the thermal pressure, which can be significant in gamma-ray burst sources.

Finally, the problem of the 'electric wiring' can also be easily solved at first glance. Due to the presence of a strong magnetic field, in all cases the Larmor radius of particles $r_{\mathrm{L}}=m c v / e B$ is always much smaller than the size $R$ of the central engine. Therefore, one can consider with good accuracy that the electric current flows along the direction of the regular magnetic field. However, here we meet the problem of current closing, since particles

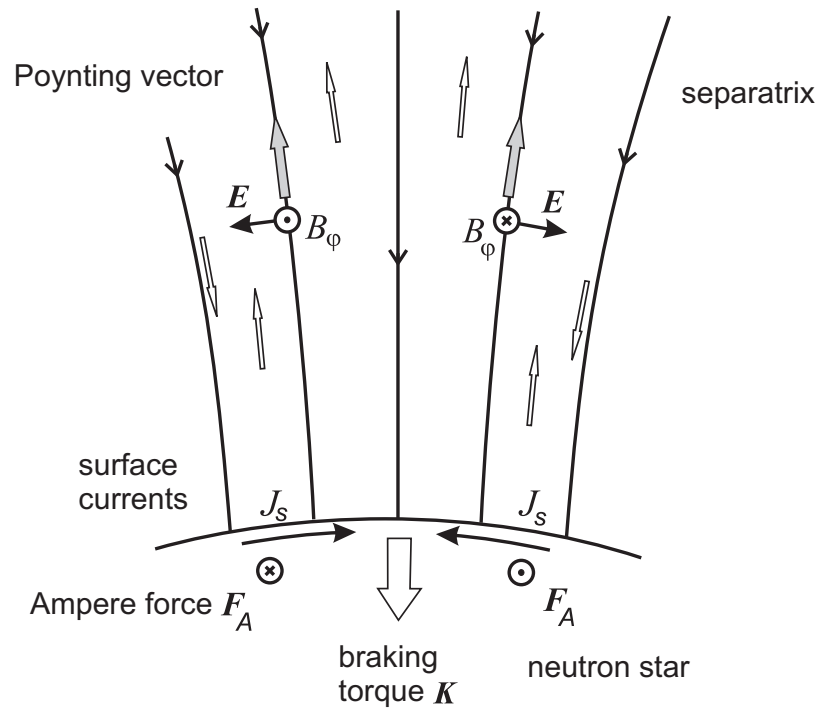

Figure 7: The structure of electric currents (contour arrows) near the polar caps of a neutron star. The Ampere force related to the surface current $\mathbf{J}_{\mathbf{s}}$, produces the torque $\mathbf{K}$ braking the neutron star rotation. Above the acceleration region, the energy flux is predominantly transported by the Poynting vector (hatched arrows).

in the region of the load must move across the magnetic field. We shall necessarily discuss this point below.

As an example, Fig. 7 illustrates how the braking occurs in an axisymmetric magnetosphere of radio pulsars. Clearly, the total current flowing out of the pulsar surface must vanish; thus, there must necessarily be a reverse current in the magnetosphere to compensate for the loss of charges from the neutron star. As a result, currents $\mathbf{J}_{\mathrm{s}}$ closing the longitudinal currents in the magnetosphere must flow over the pulsar surface. The ponderomotive action of these currents must brake the rotation of the radio pulsars $[3,56]$.

Thus, the problem of the magnitude of potential difference is solved quite easily. But the problem of the load that determines the current $I$ and, hence, the energy losses, proved much more difficult. A long way had to be covered in order to solve it, and this, essentially, will be discussed in this review. Nevertheless, we shall go somewhat ahead and give here the preliminary estimates confirming the applicability of the discussed mechanism. As shown below, a good estimate of the electric current density is given by the expression

$$
j_{\mathrm{GJ}}=\rho_{\mathrm{GJ}} c,
$$

where

$$
\rho_{\mathrm{GJ}}=-\frac{\boldsymbol{\Omega} \cdot \mathbf{B}}{2 \pi c}
$$

is the electric charge density that is needed for the electric field in the rotating reference frame to vanish. Formula (9) can be easily derived from relation (4). It was 
first applied to the neutron star magnetosphere in the pioneering paper by $\mathrm{P}$ Goldreich and $\mathrm{W} \mathrm{H}$ Julian [3], so the charge density (9) is usually called the Goldreich density.

Clearly, the total electric current circulating in the magnetosphere of the central engine can be conveniently written out in the form

$$
I_{\text {tot }}=i_{0} I_{\mathrm{GJ}} .
$$

Here $i_{0}$ is the dimensionless current, and $I_{\mathrm{GJ}}=\pi R_{0}^{2} c \rho_{\mathrm{GJ}}$, i.e., for the case $\rho_{\mathrm{GJ}} \approx$ const we obtain

$$
I_{\mathrm{GJ}}=\frac{\Omega B_{0} R_{0}^{2}}{2} .
$$

Finally, $R_{0}$ is again the size of the working area on the central engine surface. For black holes we can set $R_{0} \approx R=r_{\mathrm{g}}$, and for neutron stars (radio pulsars) it must be on the order of the radius of a polar cap from which magnetic field lines can go beyond the light cylin$\operatorname{der} R_{\mathrm{L}}=c / \Omega$. Indeed, inside the closed magnetosphere, by virtue of the remarkable Ferraro isorotation law, the plasma starts rotating with the star as a solid body, and, hence, this region cannot work as a unipolar inductor. The working area will include only the region of open field lines, inside which the plasma rotational velocity can be different from that of the star. As a result, for the dipole magnetic field we obtain

$$
R_{0} \approx R\left(\frac{\Omega R}{c}\right)^{1 / 2} .
$$

For relativistic strongly magnetized wind it is natural to assume that

$$
i_{0} \approx 1,
$$

which corresponds to a free plasma outflow with the velocity $c$. As we shall see, this estimate is indeed correct. Therefore, the total energy losses can be estimated as

$$
W_{\text {tot }} \approx\left(\frac{\Omega R_{0}}{c}\right)^{2} B_{0}^{2} R_{0}^{2} c .
$$

In consequence of this, as shown in Table 1, the unipolar inductor model allows us to explain both the total energy release $W_{\text {tot }}$ and the time of activity of compact sources, $\tau_{\mathrm{D}}=\mathcal{E}_{\text {kin }} / W_{\text {tot }}$. As mentioned above, for radio pulsars estimate (14) with account for relation (12) coincides to within an order of magnitude with the magnetodipole losses (3).

For nonrelativistic outflows, estimate (13) is incorrect, and, as a detailed analysis shows, $i_{0} \gg 1$ [9]. For a sufficiently rapid rotation with $\Omega>\Omega_{\mathrm{cr}}$, where

$$
\Omega_{\mathrm{cr}}=\frac{v_{\text {in }}}{R_{0}}\left(\frac{4 \pi \rho_{\mathrm{in}} v_{\mathrm{in}}^{2}}{B_{0}^{2}}\right)^{1 / 2} \sim 10^{-6} \mathrm{~s}^{-1},
$$

we have

$$
i_{0} \approx \frac{c}{v_{\text {in }}}\left(\frac{\Omega_{\mathrm{F}}}{\Omega_{\mathrm{cr}}}\right)^{-2 / 3},
$$

and the dimensionless current for slow rotation limit takes the form

$$
i_{0} \approx \frac{c}{v_{\text {in }}} .
$$

Here $\rho_{\text {in }}$ is the density of the outflowing matter near the surface of the star, and $v_{\text {in }}$ is the characteristic velocity of the outflow along the jet axis. As a result, the total energy losses for rapidly rotating stars can be expressed through the directly observed quantities:

$$
W_{\text {tot }} \approx \Omega^{4 / 3} \Psi_{\text {tot }}^{4 / 3} \dot{M}^{1 / 3},
$$

i.e., through the total magnetic flux $\Psi_{\text {tot }}=\pi R_{0}^{2} B_{0}$, the rotational angular velocity $\Omega$, and the mass loss rate in the jet $\dot{M}$. For the parameters typical in young stars we have

$$
\begin{gathered}
W_{\text {tot }} \sim 10^{36}\left(\frac{P}{10^{6} \mathrm{~s}}\right)^{4 / 3}\left(\frac{B_{\text {in }}}{10^{3} \mathrm{G}}\right)^{4 / 3} \\
\left(\frac{R_{\text {in }}}{10^{11} \mathrm{~cm}}\right)^{8 / 3}\left(\frac{\dot{M}}{10^{-9} M_{\odot} \mathrm{yr}^{-1}}\right)^{1 / 3} \operatorname{erg~s}^{-1} .
\end{gathered}
$$

It is seen that this value is indeed close to the energy losses from young stellar objects. Thus, the unipolar inductor model allows us to explain the main jet characteristics for nonrelativistic sources, too.

Interestingly, the knowledge of the total energy losses $W_{\text {tot }}$ immediately allows the total longitudinal electric current the circulating in the magnetosphere to be estimated. Indeed, by comparing expressions (11) and (14), we straightforwardly obtain

$$
I \approx i_{0} c^{1 / 2} W_{\text {tot }}^{1 / 2} .
$$

The characteristic amplitudes of currents are also collated in Table 1.

\subsection{Grad-Shafranov equation method}

The Grad-Shafranov equation method lies at the heart of the analytical theory which, in our opinion, is able to quite successfully describe the main properties of active compact astrophysical sources. Simply speaking, this approach describes axisymmetric stationary flows in the framework of ideal magnetohydrodynamics. This approximation is based on the assumption of a high conductivity of the plasma that fills the magnetosphere of the central engine (the high energy release guarantees a high degree of ionization of matter, and the effective production of electron-positron pairs in the vicinity of black holes). Moreover, most of the sources discussed above (except for radio pulsars) can be considered to a good approximation as axisymmetric and stationary.

The attractiveness of this approach is related to the fact that there are quite a lot of integrals of motion in stationary ideal magnetohydrodynamics, i.e., quantities which are conserved along particle trajectories. This immediately provides us with important information without complicated calculations. Indeed, to determine the 
Table 1: Parameters of the central engine: AGN — active galactic nucleus, GRB - gamma-ray burst, $\mu$ QSO microquasar, PSR — radio pulsar, msPSR — millisecond radio pulsar, and YSO — young stellar object.

\begin{tabular}{|l|c|c|c|c|c|c|}
\hline & AGN & GRB & $\mu$ QSO & PSR & msPSR & YSO \\
\hline Mass $M$ in $M_{\odot}$ & $10^{6}-10^{9}$ & $\sim 10$ & $\sim 10$ & $\approx 1.4$ & $\approx 1.4$ & $\sim 10$ \\
\hline Radius $R, \mathrm{~cm}$ & $10^{11}-10^{14}$ & $\sim 10^{6}$ & $\sim 10^{6}$ & $\sim 10^{6}$ & $\sim 10^{6}$ & $\sim 10^{11}$ \\
\hline Working radius $R_{0}$ & $\sim R$ & $\sim R$ & $\sim R$ & $(\Omega R / c)^{1 / 2} R$ & $(\Omega R / c)^{1 / 2} R$ & $\sim R$ \\
\hline Period $P$ & $10-10^{3} \mathrm{~s}$ & $\sim 1 \mathrm{~ms}$ & $\sim 1 \mathrm{~ms}$ & $\sim 1 \mathrm{~s}$ & $1.39-10 \mathrm{~ms}$ & $1-10 \mathrm{~d}$ \\
\hline$\Omega R / c$ & $\geq 0.1$ & $\geq 0.1$ & $\geq 0.1$ & $\sim 10^{-4}$ & $\sim 10^{-1}$ & $\sim 10^{-5}$ \\
\hline Magnetic field $B_{0}, \mathrm{G}$ & $10^{3}-10^{4}$ & $\sim 10^{15}$ & $\sim 10^{10}$ & $\sim 10^{12}$ & $\sim 10^{8}$ & $\sim 10^{3}$ \\
\hline Energy storage $\mathcal{E}_{\text {kin }}, \mathrm{erg}$ & $10^{58}-10^{61}$ & $\sim 10^{52}$ & $\sim 10^{52}$ & $10^{44}-10^{46}$ & $\sim 10^{51}$ & $\sim 10^{44}$ \\
\hline Dimensionless current $i_{0}$ & 1 & 1 & 1 & 1 & 1 & $\sim c / v_{\text {in }}$ \\
\hline Power $W_{\text {tot }}$, erg s s $^{-1}$ & $10^{42}-10^{45}$ & $10^{51}-10^{52}$ & $\sim 10^{38}$ & $10^{31}-10^{34}$ & $10^{34}-10^{35}$ & $\sim 10^{35}$ \\
\hline Lifetime $\tau_{\mathrm{D}}$, yr & $\sim 10^{7}$ & $\sim 10^{-6}$ & $\sim 10^{4}$ & $10^{6}-10^{7}$ & $10^{8}-10^{9}$ & $\sim 10^{4}$ \\
\hline Current $I, \mathrm{CGSE}$ & $10^{26}-10^{28}$ & $\sim 10^{31}$ & $\sim 10^{25}$ & $10^{21}-10^{22}$ & $\sim 10^{22}$ & $\sim 10^{25}$ \\
\hline
\end{tabular}

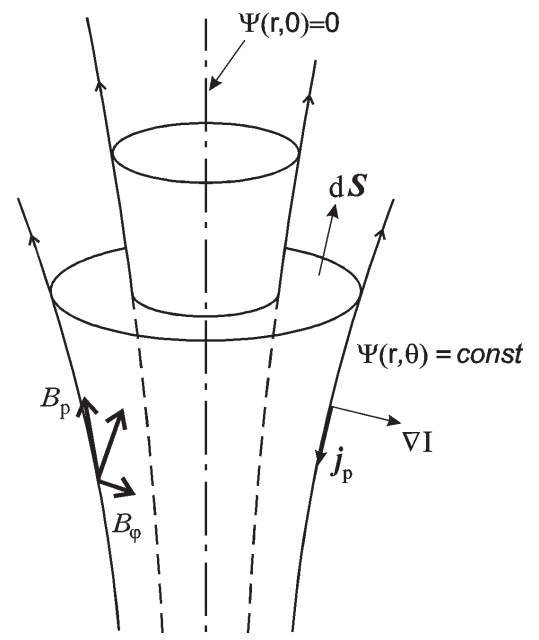

Figure 8: Axisymmetric magnetic surfaces $\Psi(r, \theta)=$ const.

height a throwing stone reaches it is not necessary to solve equations of its motion: it is sufficient to apply the energy conservation law.

In the axisymmetric case, as illustrated in Fig. 8, the magnetic field vectors must lie on the magnetic surfaces which can be easily parametrized using the magnetic flux function $\Psi(r, \theta)$ that determines the magnetic field

$$
\mathbf{B}=\frac{\nabla \Psi \times \mathbf{e}_{\varphi}}{2 \pi \varpi}-\frac{2 I}{c \varpi} \mathbf{e}_{\varphi} .
$$

Here $\varpi=r \sin \theta$ is the distance from the rotational axis, and the numerical coefficient in the first term is chosen such that the function $\Psi(r, \theta)$ indeed coincides with the magnetic flux passing through a circle $r, \theta, 0<\varphi<2 \pi$. As for the quantity $I(r, \theta)$, it represents the total electric current flowing through the same circle. It is easy to check that the following important properties are satisfied.
1. At all times $\mathrm{d} \Psi=\mathbf{B} \cdot \mathrm{d} \mathbf{S}$ (d $\mathbf{S}$ is the surface element). Therefore, the function $\Psi(r, \theta)$ indeed bears the sense of the magnetic flux.

2. Since the poloidal part of the magnetic field in formula (21) can be written out as $(2 \pi)^{-1} \nabla \Psi \times \nabla \varphi$, the condition $\nabla \cdot \mathbf{B}=0$ is automatically satisfied. Thus, three components of the magnetic field are completely determined by two scalar functions $\Psi(r, \theta)$ and $I(r, \theta)$.

3. For the same reason it is clear that the condition B $\cdot \nabla \Psi=0$ will be satisfied for axisymmetric flows. Therefore, the lines $\Psi(r, \theta)=$ const define the form of magnetic surfaces. As a result, the integrals of motion should depend on only one scalar function, $\Psi(r, \theta)$.

Let us now understand which integrals of motion appear in the case of axisymmetric stationary flows. Incidentally, the very structure of the Grad-Shafranov equation method will become clear. For simplicity, let us consider first a purely hydrodynamic flow. In this case, in analogy with relation (21), it is necessary to introduce the function $\Phi(r, \theta)$ of hydrodynamic flux defined as

$$
\rho \mathbf{v}_{\mathrm{p}}=\frac{\nabla \Phi \times \mathbf{e}_{\varphi}}{2 \pi r \sin \theta},
$$

where hereinafter the subscript 'p' will correspond to the poloidal [i.e., lying in the $(r, \theta)$ plane] components of vectors.

In hydrodynamics, there are five scalar equations (the mass continuity equation, three components of the momentum Euler equation, and the energy conservation equation) for five unknown quantities - three velocity components, and two thermodynamic functions. However, due to the axial symmetry, stationarity, and ideality of the flow three of five equations can be represented in the form $(\mathbf{v} \nabla) \mathcal{I}^{(i)}=0$, which means that integrals $\mathcal{I}^{(i)}$ must be constant on the surfaces $\Phi(r, \theta)=$ const. As is well known, these integrals include the energy (Bernoulli 
integral) $E_{\mathrm{n}}$, the specific angular momentum $L_{\mathrm{n}}$, and the entropy $s$ :

$$
\begin{aligned}
E_{\mathrm{n}} & =E_{\mathrm{n}}(\Phi)=\frac{v^{2}}{2}+w(\rho, s)+\varphi_{\mathrm{g}}, \\
L_{\mathrm{n}} & =L_{\mathrm{n}}(\Phi)=v_{\varphi} r \sin \theta, \\
s & =s(\Phi) .
\end{aligned}
$$

Here, $\varphi_{\mathrm{g}}$ is the gravitational potential, $w(\rho, s)$ is the specific enthalpy, and the subscript 'n' corresponds to nonrelativistic quantities, while Bernoulli integral $E_{\mathrm{n}}(\Phi)$ corresponds to the projection of the Euler equation (i.e., the equation of the force balance) onto the direction along the poloidal velocity $\mathbf{v}_{\mathrm{p}}$, and the angular momentum $L_{\mathrm{n}}(\Phi)$ represents the projection onto the unit vector $\mathbf{e}_{\varphi}$. The remaining two first-order equations can be reduced to one second-order equation for the flux function $\Phi(r, \theta)$. It is clear that this equation will describe the force balance in the direction perpendicular to surfaces $\Phi(r, \theta)=$ const. In the compact form, it can be written out as

$$
\begin{aligned}
& -\varpi^{2} \nabla_{k}\left(\frac{1}{\varpi^{2} \rho} \nabla^{k} \Phi\right)-4 \pi^{2} \rho L_{\mathrm{n}} \frac{\mathrm{d} L_{\mathrm{n}}}{\mathrm{d} \Phi} \\
& +4 \pi^{2} \varpi^{2} \rho \frac{\mathrm{d} E_{\mathrm{n}}}{\mathrm{d} \Phi}-4 \pi^{2} \varpi^{2} \rho \frac{T}{m_{\mathrm{p}}} \frac{\mathrm{d} s}{\mathrm{~d} \Phi}=0 .
\end{aligned}
$$

It should be noted that we deliberately defined the enthalpy $w$ as a function of density $\rho$ and entropy $s$. The point is that Bernoulli equation with the use of definition (22) can be recast into the form

$$
E_{\mathrm{n}}=\frac{(\nabla \Phi)^{2}}{8 \pi^{2} \varpi^{2} \rho^{2}}+\frac{1}{2} \frac{L_{\mathrm{n}}^{2}}{\varpi^{2}}+w(\rho, s)+\varphi_{\mathrm{g}} .
$$

One can see that Bernoulli equation written in this form, in addition to the integrals of motion and the flux function $\Phi(r, \theta)$, contains only the density $\rho$. Consequently, it indirectly defines the density $\rho$ though the flux function $\Psi$ and integrals of motion:

$$
\rho=\rho\left(\nabla \Phi ; E_{\mathrm{n}}, L_{\mathrm{n}}, s ; r, \theta\right) .
$$

This implies that after substituting Eqn (28) into Eqn (26), the latter will contain only one unknown flux function $\Phi(r, \theta)$ and three integrals of motion depending on it.

Such an equation can also be written out in the framework of ideal magnetohydrodynamics, too. In this case, however, not three but five integrals of motion exist. Two additional integrals come from the freezing-in condition

$$
\mathbf{E}+\mathbf{v} \times \mathbf{B} / c=0 .
$$

Indeed, from condition (29) follows that the electric field is perpendicular to the magnetic field. In the axisymmetric case this means that magnetic surfaces $\Psi(r, \theta)=$ const will be equipotential ones. This condition can be conveniently rewritten in the form

$$
\Omega_{\mathrm{F}}=\Omega_{\mathrm{F}}(\Psi),
$$

where the scalar quantity $\Omega_{\mathrm{F}}$ determines the electric field according to the definition

$$
\mathbf{E}=-\frac{\Omega_{\mathrm{F}}}{2 \pi c} \nabla \Psi .
$$

This is related to the fact that:

- in the axisymmetric case $(\partial / \partial t=0)$ Maxwell equation $\nabla \times \mathbf{E}=0$ leads to the condition $E_{\varphi}=0$,

- the freezing-in condition yields $E_{\|}=0$,

- the definition [31] together with Maxwell equation $\nabla \times \mathbf{E}=0$ leads to the condition $\nabla \Omega_{\mathrm{F}} \times \nabla \Psi=0$, where relation (30) comes from.

Function $\Omega_{\mathrm{F}}$ introduced in this way bears the meaning of the angular velocity of particles (more precisely, the motion of particles is the sum of rotation with the angular velocity $\Omega_{\mathrm{F}}$ and sliding along the magnetic field). Condition (30) represents the Ferraro isorotation law [83], according to which the angular velocity of particle motion relative to the magnetic field must be constant on axisymmetric magnetic surfaces.

On the other hand, the freezing-in plasma condition implies that plasma velocity vectors $\mathbf{v}$ have also to lie on the magnetic surfaces, i.e., the flux of matter does not intersect the boundaries of the magnetic surfaces. This means that the particle flux function $\Phi(r, \theta)$ must be a function of the magnetic flux $\Psi(r, \theta)$. This fact allows us to introduce one more integral of motion

$$
\eta_{\mathrm{n}}(\Psi)=\frac{\mathrm{d} \Phi}{\mathrm{d} \Psi},
$$

which, as evidenced by the foregoing, bears the sense of the ratio of the particle flux to the magnetic field flux. Correspondingly, the poloidal velocity of matter can be written as

$$
\mathbf{v}_{\mathrm{p}}=\frac{\eta_{\mathrm{n}}}{\rho} \mathbf{B}_{\mathrm{p}} .
$$

As for the energy and angular momentum integrals (which in the nonrelativistic case are usually considered as functions of the particle flux), they now take the form

$$
\begin{aligned}
& E_{\mathrm{n}}(\Phi)=\frac{\Omega_{\mathrm{F}} I}{2 \pi \eta_{\mathrm{n}} c}+\frac{v^{2}}{2}+w+\varphi_{\mathrm{g}}, \\
& L_{\mathrm{n}}(\Phi)=\frac{I}{2 \pi \eta_{\mathrm{n}} c}+v_{\varphi} r \sin \theta,
\end{aligned}
$$

respectively. The entropy $s(\Psi)$ is ones again the one more (fifth) invariant. It is clear that both particles and electromagnetic field contribute to the energy and angular momentum, and, as can be easily checked, the term $\Omega_{\mathrm{F}} I / 2 \pi \eta_{\mathrm{n}} c$ corresponds simply to the Poynting vector flux.

Next, Bernoulli equation (34) can now be rewritten in the form

$$
\begin{array}{r}
\frac{\mathcal{M}^{4}}{64 \pi^{4} \eta_{\mathrm{n}}^{2}}(\nabla \Psi)^{2}=2 \varpi^{2}\left(E_{\mathrm{n}}-w-\varphi_{\mathrm{g}}\right) \\
-\frac{\left(\Omega_{\mathrm{F}} \varpi^{2}-L_{\mathrm{n}} \mathcal{M}^{2}\right)^{2}}{\left(1-\mathcal{M}^{2}\right)^{2}}-2 \varpi^{2} \Omega_{\mathrm{F}} \frac{L_{\mathrm{n}}-\Omega_{\mathrm{F}} \varpi^{2}}{1-\mathcal{M}^{2}}
\end{array}
$$


where

$$
\mathcal{M}^{2}=\frac{4 \pi \eta_{\mathrm{n}}^{2}}{\rho} .
$$

The quantity $\mathcal{M}^{2}$ is the square of the Mach number of the poloidal velocity $v_{\mathrm{p}}$ relative to the poloidal component of the Alfvén velocity:

$$
v_{\mathrm{Ap}}=\frac{B_{\mathrm{p}}}{\sqrt{4 \pi \rho}}
$$

i.e., $\mathcal{M}^{2}=v_{\mathrm{p}}^{2} / v_{\mathrm{Ap}}^{2}$. It should be recalled that the specific enthalpy $w$ in equation (36) must be considered as a function of entropy $s$, as well as of the Mach number $\mathcal{M}^{2}$ and the integral $\eta_{\mathrm{n}}$. The corresponding relationship has the form

$$
\nabla w=c_{\mathrm{s}}^{2}\left(2 \frac{\nabla \eta_{\mathrm{n}}}{\eta_{\mathrm{n}}}-\frac{\nabla \mathcal{M}^{2}}{\mathcal{M}^{2}}\right)+\left[\frac{1}{\rho}\left(\frac{\partial P}{\partial s}\right)_{n}+\frac{T}{m_{\mathrm{p}}}\right] \nabla s .
$$

In consequence, as in the hydrodynamic limit, Bernoulli equation allows one to determine, albeit indirectly, the quantity $\mathcal{M}^{2}$ via the magnetic flux $\Psi(r, \theta)$ and five integrals of motion:

$$
\mathcal{M}^{2}=\mathcal{M}^{2}\left(\nabla \Psi ; E_{\mathrm{n}}, L_{\mathrm{n}}, s, \eta_{\mathrm{n}}, \Omega_{\mathrm{F}} ; r, \theta\right) .
$$

As for the projection of the force balance onto the direction perpendicular to the magnetic surfaces, it can be written in the form

$$
\begin{aligned}
& \frac{1}{16 \pi^{3} \rho} \nabla_{k}\left(\frac{1-\mathcal{M}^{2}}{\varpi^{2}} \nabla^{k} \Psi\right)+\frac{\mathrm{d} E_{\mathrm{n}}}{\mathrm{d} \Psi} \\
& +\frac{\Omega_{\mathrm{F}} \varpi^{2}-L_{\mathrm{n}}}{1-\mathcal{M}^{2}} \frac{\mathrm{d} \Omega_{\mathrm{F}}}{\mathrm{d} \Psi}+\frac{1}{\varpi^{2}} \frac{\mathcal{M}^{2} L_{\mathrm{n}}-\Omega_{\mathrm{F}} \varpi^{2}}{1-\mathcal{M}^{2}} \frac{\mathrm{d} L_{\mathrm{n}}}{\mathrm{d} \Psi} \\
& +\left[2\left(E_{\mathrm{n}}-w-\varphi_{\mathrm{g}}\right)+\frac{\Omega_{\mathrm{F}}^{2} \varpi^{4}-2 \Omega_{\mathrm{F}} L_{\mathrm{n}} \varpi^{2}+\mathcal{M}^{2} L_{\mathrm{n}}^{2}}{\varpi^{2}\left(1-\mathcal{M}^{2}\right)}\right] \\
& \times \frac{1}{\eta_{\mathrm{n}}} \frac{\mathrm{d} \eta_{\mathrm{n}}}{\mathrm{d} \Psi}-\frac{T}{m_{\mathrm{p}}} \frac{\mathrm{d} s}{\mathrm{~d} \Psi}=0 .
\end{aligned}
$$

Now the structure of the treatment considered here becomes clear. Equation (41) jointly with Bernoulli equation (36) determines the value of the magnetic flux $\Psi(r, \theta)$. Then, again using Bernoulli equation, one can determine the value of the Mach number $\mathcal{M}$ at each point. After that it turned out that the number of integrals of motion is enough for determining all other quantities from simple algebraic equations. For example, one arrives at [84]

$$
\begin{aligned}
\frac{I}{2 \pi} & =c \eta_{\mathrm{n}} \frac{L_{\mathrm{n}}-\Omega_{\mathrm{F}} \varpi^{2}}{1-\mathcal{M}^{2}} \\
v_{\varphi} & =\frac{1}{\varpi} \frac{\Omega_{\mathrm{F}} \varpi^{2}-L_{\mathrm{n}} \mathcal{M}^{2}}{1-\mathcal{M}^{2}}
\end{aligned}
$$

and, respectively, $\rho=4 \pi \eta_{\mathrm{n}}^{2} / \mathcal{M}^{2}$. This is essentially the main attractiveness of the approach we discuss. Sometimes, as we shall see, the key properties can be directly

\footnotetext{
${ }^{1}$ Unfortunately, in monograph [9] terms $-w-\varphi_{\mathrm{g}}$ in Eqn (4.102) were discarded.
}

obtained from algebraic relations. In other words, by making sufficiently reasonable assumptions about the structure of the flow [i.e., about flux function $\Psi(r, \theta)$ ], it is possible not to solve equation (41) at all and to analyze only algebraic, albeit indirect, relations.

The full version of the nonrelativistic equation containing all five invariants was first formulated by L S Solov'ev in 1963 in the third volume of the Reviews of Plasma Physics [85]. Being virtually unknown for astrophysicists, this equation was later reformulated anew several times [86-88]. For this reason, in particular, to date there has been no unique system of notations, so sometimes it is difficult to compare the results of different studies. In the literature, equations of this type are commonly called Grad-Shafranov equations, which were formulated at the end of the 1950s in relation to controlled thermonuclear fusion [89, 90], although the hydrodynamic version of this equation was known even earlier (see, for example, Ref. [91]). Similar equations going back to the classical Tricomi equation, were discussed as early as the beginning of the twentieth century in the context of transonic hydrodynamic flows [92, 93].

For simplicity, we have written out above equations only for the nonrelativistic case. However, it was not too difficult to obtain the corresponding equations both for the relativistic case [94] and for flows in the vicinities of nonrotating [95] and rotating [96, 97] black holes, since the Kerr metric is axisymmetric and stationary. It is these relativistic equations that we shall discuss below. In the main text we shall try to formulate sufficiently simple asymptotic expressions by focusing on the qualitative description of the flow properties. Sufficiently lengthy full equations are presented in the Appendix. Here, we shall restrict ourselves by writing out additionally the integrals of motion for relativistic flows in flat space.

Clearly, magnetic surfaces remain equipotential in the relativistic case, too. Thus, the angular frequency $\Omega_{\mathrm{F}}$ in definition (31) remains the integral of motion. As for the integrals of energy $E$ and the $z$-component of the angular momentum $L$, now they should be written out as $2^{2}$

$$
\begin{aligned}
& E=E(\Psi)=\frac{\Omega_{\mathrm{F}} I}{2 \pi}+\gamma \mu \eta c^{2}, \\
& L=L(\Psi)=\frac{I}{2 \pi}+\mu \eta \varpi u_{\varphi} c .
\end{aligned}
$$

Here $\mathbf{u}$ is the spatial part of the four-velocity vector $\left(\gamma=\sqrt{u^{2}+1}\right.$ is the Lorentz factor), and

$$
\mu \approx m_{\mathrm{p}} c^{2}+m_{\mathrm{p}} w+\ldots
$$

is the relativistic enthalpy including the rest mass of particles. Finally, the relativistic integral of motion $\eta$ is now determined from the condition

$$
\mathbf{u}_{\mathrm{p}}=\frac{\eta}{n} \mathbf{B}_{\mathrm{p}}
$$

\footnotetext{
${ }^{2}$ To avoid misunderstanding, from now on the electric field $\mathbf{E}$ will always be boldfaced.
} 
where $n$ is the particle number density, and hereinafter all thermodynamic functions will be defined in the comoving reference frame. Thus, for relativistic flows where $\left|\mathbf{u}_{\mathrm{p}}\right| \approx \gamma$ we simply have

$$
\eta=\frac{n^{(\mathrm{lab})}}{B_{\mathrm{p}}} .
$$

It should be noted that the relativistic and nonrelativistic integrals of motion have different dimensions, since the relativistic integrals are normalized not on the unit matter flux $d \Phi$ but on the unit magnetic flux $d \Psi$.

It is evident that again both the energy flux and the angular momentum flux include the contributions from the electromagnetic field and particles, with the electromagnetic contribution [accurate up to an additional factor $\eta(\Psi)$ ] fully coinciding with that obtained in the nonrelativistic limit. In the general magnetohydrodynamic case, the total energy and angular momentum losses, $W_{\text {tot }}$ and $K_{\text {tot }}$, will be determined by the relationships

$$
\begin{aligned}
& W_{\text {tot }}=\frac{1}{c} \int_{0}^{\Psi_{\max }} E(\Psi) \mathrm{d} \Psi, \\
& K_{\text {tot }}=\frac{1}{c} \int_{0}^{\Psi_{\max }} L(\Psi) \mathrm{d} \Psi,
\end{aligned}
$$

respectively.

\subsection{Supersonic flows}

3.3.1. The model. In order to clearly understand the main features of the model considered here, it is convenient from the very beginning to analyze a sufficiently simple geometry of magnetic surfaces, and to formulate basic parameters characterizing the flow. Figure 9 demonstrates the simplestsplit monopole model of the magnetized wind [33], which during many years served as the 'hydrogen atom' for all researchers who studied the nature of the activity of galactic nuclei, gamma-ray bursts, and microquasars. Notably, most analytical results were obtained exactly for such flows.

It is assumed in the framework of this model that the 'central engine' involves a compact object (neutron star or black hole) and an accretion disc which separates the converging and diverging magnetic field fluxes. The accretion disc here is nedeed both to separate the oppositely directed magnetic field fluxes and, in the case of a black-hole magnetosphere, to generate the regular poloidal magnetic field (it will be produced by the toroidal currents flowing in the disc). In the absence of the accretion disc, a black hole, as is well known, cannot have the proper magnetic field (the so-called 'no-hair theorem' [18]). In addition, poloidal currents will also flow in the disc, closing the bulk currents flowing in the magnetosphere. Notice that a similar configuration with a disc separating magnetic field fluxes far from the neutron star also emerges in many models of radio pulsars [98 104], because it is natural to assume that at large distances the flow becomes quasispherical.

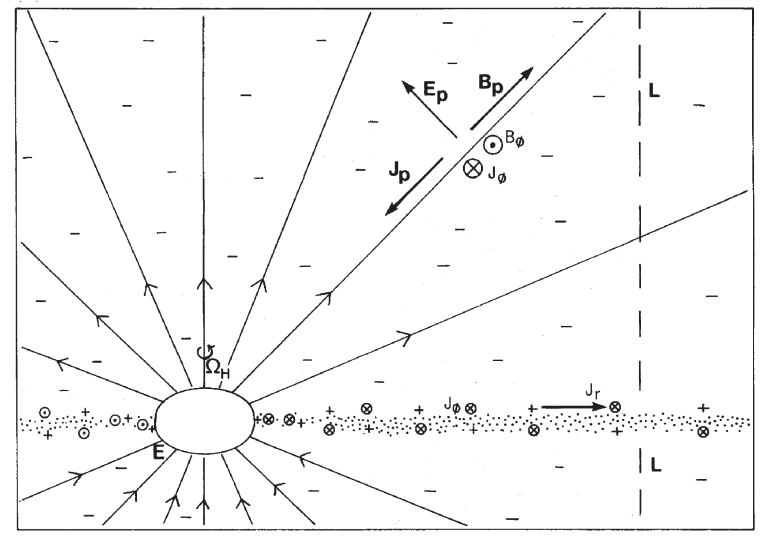

Figure 9: The structure of electromagnetic fields for the split monopole magnetic field near a slowly rotating black hole [33]. Currents flowing in the highly conducting disc in the equatorial plane provide both the poloidal magnetic field jump and the closure of bulk currents flowing out the upper and bottom hemispheres.

Next, it is important that the crossed fields $E_{\theta}$ and $B_{\varphi}$ form the electromagnetic energy flux (the Poynting vector flux), which is directed along the magnetic surfaces. It should be stressed that this energy is transferred at the zero frequency, so the electromagnetic field that carries the energy is not an electromagnetic wave in the usual sense. The electromagnetic energy flux therefore appears only due to the longitudinal current generating the toroidal magnetic field; in the absence of particles, such energy release becomes impossible. The plasma also moves along the magnetic surfaces, so the sum of their energy fluxes is the integral of motion. On the other hand, the longitudinal current $I$ is not the integral of motion, so the MHD approximation we are considering allows, in principle, describing the current closure phenomenon. However, the magnetic surfaces here remain equipotential. Therefore, such flows can carry high electric voltages over large distances from the central engine. This should always be borne in mind when discussing the interaction of a magnetized wind with the surrounding medium. For example, this effect must be taken into account in close binary systems with radio pulsars.

As for the main dimensionless quantities characterizing the flow, they include the magnetization parameter $\sigma$, the particle production multiplicity $\lambda$, and the compactness parameter $l_{a}$. The magnetization parameter $\sigma$ shows by how much the electromagnetic energy flux near the central engine can exceed the particle energy flux. Thus, as seen from the definition of the energy integral (44), it can be more conveniently defined for relativistic flows as

$$
\sigma=\left(\frac{E}{\mu \eta c^{2}}\right)_{\text {max }},
$$

where the maximal value is chosen for all magnetic surfaces. As a result, the value of $\sigma$ corresponds to the max- 
imal Lorentz factor of the plasma that can be reached in the case where all the electromagnetic field energy is transferred to the particles. In other words, $\sigma$ is the maximum Lorentz factor that can be achieved in the magnetized wind. Of course, here the mean hydrodynamic energy of the plasma flowing out is assumed. In particular, for the split monopole magnetic field (for which this quantity was first introduced by F C Michel [4] in 1969), we obtain

$$
\sigma=\frac{\Omega^{2} \Psi_{\text {tot }}}{8 \pi^{2} c^{2} \mu \eta}
$$

Correspondingly, for the nonrelativistic flow it is convenient to use the quantity

$$
\sigma_{\mathrm{n}}=\frac{\Omega^{2} \Psi_{\mathrm{tot}}}{8 \pi^{2} v_{\mathrm{in}}{ }^{3} \eta_{\mathrm{n}}}
$$

where $v_{\text {in }}$ is the velocity of matter flow along the jet axis. It is easy to check that the strong magnetization condition $\Omega>\Omega_{\mathrm{cr}}$ (15) coincides with the condition $\sigma_{\mathrm{n}}>1$.

Recall that we are mainly interested in strongly magnetized flows, i.e., flows in which the main energy flux near the central engine is due to the Poynting vector flux $\Omega_{\mathrm{F}} I / 2 \pi$.. In the opposite case, the flow will be only slightly different from the hydrodynamic outflow. Using definition (51), this condition can be rewritten as

$$
\gamma_{\text {in }} \ll \sigma,
$$

where $\gamma_{\text {in }}$ is the injection Lorentz factor. As we shall see, the magnetization parameter $\sigma$ is the key parameter determining the basic features of the flow.

Next, to find the ejected plasma density, it is convenient to introduce the dimensionless particle production multiplicity $\lambda$

$$
\lambda=\frac{n^{(\mathrm{lab})}}{n_{\mathrm{GJ}}},
$$

where $n_{\mathrm{GJ}}=\left|\rho_{\mathrm{GJ}}\right| / e$. Such a definition is connected with the fact that, as we shall show below, both in pulsar magnetospheres and in black hole magnetospheres the densest is the secondary electron-positron plasma generated either due to conversion of hard gamma-quanta in the magnetic field or due to gamma-ray collisions with thermal photons [33, 105]. However, hard gamma-quanta must be emitted in both cases by primary particles whose density is supposed to be close to the Goldreich density. The convenience of the particle production multiplicity $\lambda$ also stems from the fact that the magnetization parameter $\sigma$ can be rewritten with its help in the form

$$
\sigma=\frac{e \Omega \Psi_{\text {tot }}}{4 \lambda m_{\mathrm{e}} c^{3}} \sim \frac{1}{\lambda}\left(\frac{W_{\mathrm{tot}}}{W_{\mathrm{A}}}\right)^{1 / 2},
$$

where $W_{\mathrm{A}}=m_{\mathrm{e}}^{2} c^{5} / e^{2} \approx 10^{17} \mathrm{erg} \mathrm{s}^{-1}$. Thus, the knowledge of two of three quantities $W_{\text {tot }}, \sigma$, and $\lambda$, allows the determination of the third one.

Finally, the compactness parameter

$$
l_{a}=\frac{\sigma_{\mathrm{T}} L_{\mathrm{tot}}}{m_{\mathrm{e}} c^{3} R}
$$

is in fact the optical depth for Thomson cross section $\sigma_{\mathrm{T}}$ at a distance $R$ from a source with the total luminosity $L_{\text {tot }}$. Below, it will be important for us that the parameter $l_{a}$ provide an upper limit of particle energy in the acceleration region. On the other hand, a large $l_{a}$ is necessary for effective particle production.

3.3.2. Singular surfaces. Singular surfaces represent the most important structural element of flows. As we shall see, it is the analysis of the conditions of the smooth passing of a flow through singular surfaces that allows sometimes rather general relationships to be obtained without solving the Grad-Shafranov equation itself. Notice from the very beginning that, for simplicity, below we shall only analyze the case of cold flows. The point here is that, at large distances thermal effects are insignificant for the polytropic index $\Gamma>1$ (pressure $\left.P \propto n^{\Gamma}\right)$. This conclusion can readily be obtained from both the Grad-Shafranov equation itself and Bernoulli equation. Indeed, from the analysis, for instance, of nonrelativistic equations (36) and (41) it follows that both the enthalpy $w=c_{\mathrm{s}}^{2} /(\Gamma-1) \propto n^{\Gamma-1}$ in Eqn (36) and the temperature $T \propto n^{\Gamma-1}$ in Eqn (41) decrease with the distance from the compact source, since for any divergent outflow the particle number density $n \rightarrow 0$ for $r \rightarrow \infty$. Therefore, the contribution from a final temperature (enthalpy, entropy) compared to the total energy $E$ and its derivative $\mathrm{d} E / \mathrm{d} \Psi$ can be neglected at large distances. Thus, it becomes clear why in the analysis of relativistic flows the final temperature effects (and, in particular, critical conditions on the slow magnetosonic surface) are usually neglected. On the other hand, the pressure can be significant for cylindrical flows, i.e., for flows in which the density does not decrease with distance from compact object $[9,106]$.

The first natural scale that emerges in the theory of relativistic winds is the light cylinder

$$
R_{\mathrm{L}}=\frac{c}{\Omega},
$$

i.e., the axial distance at which solid-body rotation together with the central object becomes impossible. It is easy to show that the light cylinder is the scale where:

1. the magnitude of the electric field becomes comparable to that of the poloidal magnetic field;

2. toroidal electric currents flowing in the magnetosphere start perturbing the poloidal manetic field of the central engine;

3. the magnitude of the toroidal magnetic field produced by the longitudinal Goldreich current becomes comparable with that of the poloidal magnetic field.

It follows from the first statement above and definitions (21) and (31) that beyond the light cylinder the electric field becomes stronger than the poloidal magnetic field. In particular, the poloidal magnetic field will decrease as $r^{-2}$ and the electric field as $r^{-1}$ for the spit 
monopole outflow shown in Fig. 9. On the other hand, freezing-in condition (29) requires that the magnetic field be stronger than the electric field. This can be possible only when a strong enough longitudinal electric current is flowing in the magnetosphere, because the toroidal magnetic field also decreases as $r^{-1}$ according to Eqn (21).

We can therefore conclude that the question as to whether or not a smooth relativistic MHD outflow $(|\mathbf{E}|<|\mathbf{B}|)$ exists beyond the light cylinder is also directly related to the question of the magnitude of the longitudinal current circulating in the magnetosphere of a compact object. Then, for currents below some critical value, a so-called light surface is bound to appear in the magnetosphere, on which the electric field matches the magnetic field $(|\mathbf{E}|=|\mathbf{B}|)$, and hence the approximation considered here itself becomes invalid. Calculations [107, 108] showed that the closure of currents occurs near this surface in the region with the thickness $\delta r \sim R_{\mathrm{L}} / \lambda$, and particles are effectively accelerated there up to energies of $\gamma \sim \sigma$.

If the longitudinal currents are sufficiently high, the smooth MHD outflow can exist beyond the light cylinder as well. The electric field there will be almost equal to the magnetic field. Indeed, as directly follows from the relativistic Bernoulli equation (A.12), in the limit $\varpi \gg R_{\mathrm{L}}$ we obtain simply

$$
\mathbf{B}^{2}-|\mathbf{E}|^{2}=\frac{B_{\varphi}^{2}}{\gamma^{2}} .
$$

Since, as can be easily checked, $B_{\mathrm{p}} \approx B_{\varphi} / x_{r}$, where $x_{r}=\Omega \varpi / c$, we can always apply the estimate

$$
B_{\varphi}^{2}-|\mathbf{E}|^{2} \leq \frac{B_{\varphi}^{2}}{\gamma^{2}}
$$

As a result, the radial drift motion in the crossed electromagnetic fields dominates in a strongly magnetized relativistic outflow beyond the light cylinder. Indeed, as can be easily verified, the Lorentz factor entering into Eqn (59) satisfies the condition $\gamma^{-2}=1-U_{\mathrm{dr}}^{2}$, where

$$
\mathbf{U}_{\mathrm{dr}}=c \frac{\mathbf{E} \times \mathbf{B}}{B^{2}} .
$$

In other words, the velocity parallel to the magnetic field does not contribute at all to the value of the Lorentz factor [109].

The fast magnetosonic surface is another important surface of the magnetized flows. It is fully equivalent to the sonic surface in the ideal hydrodynamics. Indeed, Bernoulli equation (27) is well known to have a singularity on the sonic surface. For example, the logarithmic derivative of density determined from Eqn (27) is written for a spherically symmetric flow in the form

$$
\eta_{1}=\frac{r}{\rho} \frac{\mathrm{d} \rho}{\mathrm{d} r}=\frac{2 v^{2}-\frac{G M}{r}}{c_{\mathrm{s}}^{2}-v^{2}}=\frac{2-\frac{G M}{r v^{2}}}{-1+\frac{c_{\mathrm{s}}^{2}}{v^{2}}}=\frac{N}{D} .
$$

\footnotetext{
${ }^{3}$ This expression corrects Eqn (4.144) from monograph [9].
}

It is obvious that derivative (62) has a singularity when the velocity of matter equals the speed of sound: $v=c_{\mathrm{s}}=c_{*}(D=0)$. This means that in order to cross the sonic surface $r=r_{*}$ smoothly, the additional condition

$$
N\left(r_{*}\right)=2-\frac{G M}{r_{*} c_{*}^{2}}=0
$$

must be satisfied. As a result, the additional critical condition (63) fixes the accretion (ejection) rate of matter [14].

The fast magnetosonic surface plays a similar role. But now it determines not the accretion or ejection rate, but the magnitude of the longitudinal current $I$ (more precisely, the integral $L$ ). It is this critical condition on this surface that shows us that in the relativistic case the longitudinal current $I$ near the central engine must be close to the Goldreich current $I_{\mathrm{GJ}}$. On the other hand, as we have already noted, for a nonrelativistic outflow $i_{0} \gg 1$, and the conditions of the smooth crossing of singular surfaces lead to relations (15)-(17) used above. Their derivation, however, is rather cumbersome, and we shall omit it here. It should only be emphasized that they can be obtained directly from an analysis of Bernoulli equation. The point is that the sonic surface is the $X$-point on the (distance $r$-velocity $v$ ) plane. That is, it is the point of crossing the roots of the algebraic Bernoulli equation. The condition of coincidence of roots of the algebraic equation puts certain bounds on the coefficients of the equation itself, which enables the magnitude of the longitudinal current to be estimated. Notice that expressions for the current formulated above were exactly obtained in Refs $[110,111]$ for the simplest split monopole geometry shown in Fig. 9 .

In a similar way, the following theorem can be proved: In a relativistic outflow near the outer fast magnetosonic surface, the energy of the particles reaches the values

$$
\begin{array}{ll}
\gamma=\left(\frac{E}{\mu \eta c^{2}}\right)^{1 / 3} \sim \sigma^{1 / 3}, & \gamma_{\text {in }} \ll \sigma^{1 / 3}, \\
\gamma=\gamma_{\mathrm{in}}, & \gamma_{\mathrm{in}} \gg \sigma^{1 / 3} .
\end{array}
$$

Thus, the fraction of energy carried by particles in the vicinity of the fast magnetosonic surface is a small fraction $\left(\sim \sigma^{-2 / 3}\right)$ of the electromagnetic energy flux for strongly magnetized outflows $\sigma \gg \gamma_{\mathrm{in}}$. The surface itself is located at the distance of

$$
\begin{gathered}
r_{\mathrm{F}} \approx\left(\frac{E}{\mu \eta c^{2}}\right)^{1 / 3} R_{\mathrm{L}} \sim \sigma^{1 / 3} R_{\mathrm{L}}, \quad \gamma_{\mathrm{in}} \ll \sigma^{1 / 3},(66) \\
r_{\mathrm{F}} \approx\left(\frac{E}{\mu \eta \gamma_{\mathrm{in}} c^{2}}\right)^{1 / 2} R_{\mathrm{L}} \sim\left(\frac{\sigma}{\gamma_{\text {in }}}\right)^{1 / 2} R_{\mathrm{L}}, \\
\gamma_{\text {in }} \gg \sigma^{1 / 3}
\end{gathered}
$$

(the first relation holds true not too close to the rotational axis). Interestingly, expression (66) is valid for both relativistic and nonrelativistic flows since it does not include in fact the speed of light $c$. 
Finally, the singularity at $A=1-\mathcal{M}^{2}=0$ that appeared in nonrelativistic equations (42), (43) suggests that the Alfvénic surface must also play an important role in the structure of magnetized flows. Its location for nonrelativistic flows can easily be estimated from the numerator of relation (42):

$$
\varpi_{\mathrm{A}}^{2}=\frac{L_{\mathrm{n}}}{\Omega_{\mathrm{F}}} .
$$

In this case, the Alfvénic surface turns out to be located close to the fast magnetosonic surface.

As to a relativistic flow (and a flat space), the corresponding condition should be written differently:

$$
A=1-\frac{\Omega_{\mathrm{F}}^{2} \varpi^{2}}{c^{2}}-\mathcal{M}^{2} .
$$

On the other hand, it is easy to check that the parameter $q=W_{\text {part }} / W_{\text {em }}$ (i.e., the particle-to-electromagnetic energy flux ratio) can be presented in the form

$$
q=\frac{\mathcal{M}^{2} c^{2}}{\Omega_{\mathrm{F}}^{2} \varpi^{2}} .
$$

Thus, in the region where the energy flux is Poyntingdominated $(q \ll 1)$, the following condition should be satisfied:

$$
\mathcal{M}^{2} \ll \frac{\Omega_{\mathrm{F}}^{2} \varpi^{2}}{c^{2}} .
$$

Consequently, the Alfvénic surface for such flows is located near the light cylinder. Hence (except for the polar region where both the Alfvénic and fast magnetosonic surfaces are close to each other), the fast magnetosonic surface for strongly magnetized flows is located $\sigma^{1 / 3}$ times further from the central engine as compared with the Alfvénic surface.

Let us remember that the Alfvénic surface in the relativistic case determines the scale on which the toroidal magnetic field becomes comparable in magnitude to the poloidal field. It is easy to check that for rapid rotation $\Omega>\Omega_{\mathrm{cr}}$ (15) a similar situation holds for nonrelativistic flows, too. As regards the electric field, it is always weaker than the magnetic field in the nonrelativistic case. Notably, that is why the light surface cannot appear in the nonrelativistic case.

The Alfvénic surface represents a higher-order singularity as against the fast magnetosonic surface. Therefore, relations (42) and (43) do not put any constraint on the integrals of motion and only determine the location of the Alfvénic surface and the magnetic field structure. In this event, however, particles can intersect the Alfvénic surface only in one direction. For example, when the central source loses its rotational energy, crossing the Alfvénic surface is possible only in the direction outward from the compact object. When the energy flux is directed toward the central engine (for instance, if it is spun up by the accreting material), the flow must also be directed toward the central engine. Certainly, this

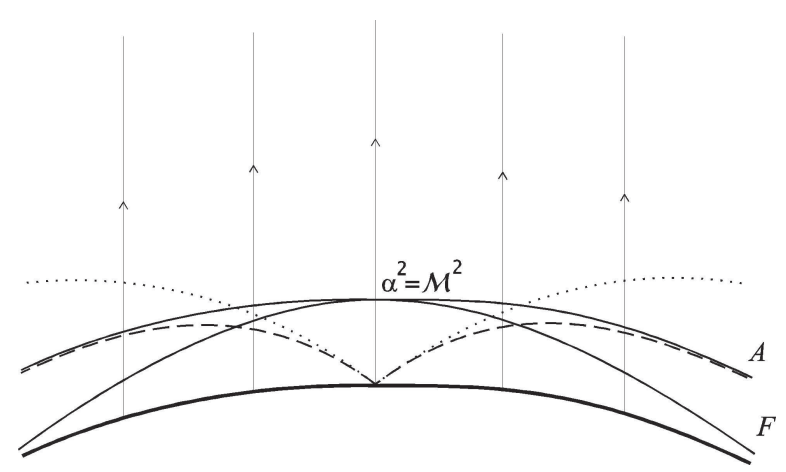

Figure 10: The location of the Alfvénic $(A)$ and fast magnetosonic $(F)$ surfaces near the black hole horizon. The dashed line shows the Alfvénic surface in the forcefree approximation (i.e., the 'light cylinder'), while the dotted line indicates the ergosphere surface. Here, $\alpha$ is the gravitational redshift (see the Appendix).

statement is invalid in the region of an accretion disc where viscosity cannot be neglected.

This statement can easily be proved in the relativistic case by recalling that the motion of particles is the sum of the drift motion in the crossed fields and the motion along the magnetic field. The condition $v<c$ that limits the longitudinal velocity puts bounds on the radial velocity of matter. This is related to the fact that the drift velocity itself becomes close to the speed of light on the Alfvénic surface. However, accretion of matter with positive energy release cannot be realized in the nonrelativistic case, either. In this event, the interaction of the supersonic accretion flow with the rotating magnetized central body would take place. A shock wave is known to be formed in such an interaction [112].

In conclusion, we should comment on the features of a black hole magnetosphere. As seen from exact expressions for the Alfvénic $A(A .13)$ and sonic $D(A .20)$ factors presented in the Appendix, a second family of singular surfaces inevitably emerges near the black hole horizon. Here, as shown in Fig. 10, the infalling matter, as in the case of the outflow, must first cross the Alfvénic surface and only then intersect the fast magnetosonic surface (recall that thermal effects are not discussed here). This is related to the fact that the strong gravitational field of the black hole forces the matter to approach the event horizon.

The appearance of the second family of singular surfaces leads to new important properties. First of all, the matter can intersect the inner Alfvénic surface only in the direction towards the black hole horizon. But this means that if the central engine loses rotational energy (and hence particles can cross the outer Alfvénic surface only in the direction away from the compact object), the plasma is bound to be generated in the magnetic field lines 'anchored' to the black hole horizon. Only in this case can electric currents appear in the black hole magnetosphere, which are necessary, as we have seen, 
to explain the observed energy release. In turn, the appearance of one more critical condition on the inner fast magnetosonic surface proved to be sufficient to determine the angular velocity $\Omega_{\mathrm{F}}$. Here, $\Omega_{\mathrm{F}}$ indeed must be close to $\Omega_{\mathrm{H}} / 2\left[\Omega_{\mathrm{H}}=\omega\left(r_{\mathrm{g}}\right)\right.$ is the angular velocity of the black hole; see the Appendix], as was understood as early as the Blandford and Znajek paper [33]. This problem has been possible to solve exactly for slow rotation in the split monopole magnetic field [113].

3.3.3. The problem setting. Before considering the main results which were obtained using the analytical theory, it is necessary to discuss the formulation of the problem. The point is that we shall primarily be interested in transonic flows, i.e., those which are subsonic near the compact object and supersonic in the wind region. Indeed, as we shall see, the distance from a central engine to singular surfaces in all compact objects are much smaller than even the transverse size of the collimated outflows. The difficulty here is that the direct problem setup itself in the framework of the GradShafranov equation method turns out to be nontrivial. For example, the second-order equation describing the two-dimensional flow in the hydrodynamic limit, when only three integrals of motion are available, requires four boundary conditions to be imposed in the transonic regime. The fifth condition is the critical condition on the sonic surface. This means that on some surface, for example, two thermodynamic functions and two velocity components must be specified. We stress that here only flows depending on two variables are considered. As discussed in detail in monograph [9], the well-known spherically symmetric flows (the Bondi accretion, the Parker ejection) are degenerate, since the structure of the flow itself is specified in them. In the general case of spherical accretion, a nonstationary solution with a shock wave appears (see, for example, Ref. [114]).

However, to determine Bernoulli integral, which is naturally needed in solving the equilibrium equation, we should specify all three components of the velocity, which is impossible since the third velocity component itself should be found from the solution. In the general case, one should set

$$
b=2+i-s^{\prime}
$$

boundary conditions, where $i$ is the number of invariants, and $s^{\prime}$ is the number of singular surfaces (and for the magnetic field lines threading the black hole horizon this number is doubled, i.e., separately for ejecting and accreting plasma). Such an internal inconsistency of this approach in the general case does not allow us to solve direct problems, namely, to determine the structure of the flow in some region using the given physical parameters on its boundary. Therefore, it is not astonishing that most researchers primarily interested in astrophysical applications already in the middle of the 1990s started addressing a totally different class of equations, namely those covering time relaxation problems, which can only be solved numerically $[79,115-119]$. However, only in the last several years has significant progress here been achieved [103, 109, 120-127], which, among other things, has confirmed many analytical results obtained before.

It should be noted that this problem does not appear in both subsonic and supersonic cases. For these flows, all necessary integrals of motion must be determined from the boundary conditions. In particular, the boundary conditions will determine in the subsonic case the longitudinal current $I$, too. This exactly corresponds to the unipolar inductor model, where the current (and hence the energy losses) is determined by the external load. Unfortunately, this ideology has also spread into the theory of magnetized winds. This is related to the fact that many results were obtained in the 1970s-1980s using the force-free approximation [i.e., when $\sigma \rightarrow \infty$ and masses of particles can be neglected]. In this approximation, the Grad-Shafranov equation becomes elliptical and, hence, the flow structure must depend on conditions at the external boundary. But the theory of pulsar magnetospheres so far has been constructed in the force-free approximation. The class of subsonic flows also includes the so-called 'magnetic tower' $[128,129]$. As this question is highly important, we shall discuss it below in more detail.

Correspondingly, the Blandford-Znajek model was also constructed in the force-free approximation, which required the boundary condition to be set on the black hole horizon. Since the electromagnetic wave (like other material bodies) can propagate near the horizon only normal to the horizon toward the black hole, the boundary condition in fact is equivalent to the Leontovich boundary condition in radio physics [130]. However, it is usually obtained by requiring the finiteness of fields in the freely falling observer's frame of reference, which yields $B_{\varphi}\left(r_{\mathrm{g}}\right)=-E_{\theta}\left(r_{\mathrm{g}}\right)$. This condition, as is well known, can be rewritten in the form of the Ohm law for the formally introduced 'surface current' [131]

$$
\mathbf{J}\left(r_{\mathrm{g}}\right)=\frac{c}{4 \pi} \mathbf{E}\left(r_{\mathrm{g}}\right),
$$

which corresponds to the universal 'internal' resistance of the battery, $\mathcal{R}=4 \pi / c=377 \Omega$. In another form, this boundary condition can be rewritten as

$$
4 \pi I(\Psi)=\left[\Omega_{\mathrm{H}}-\Omega_{\mathrm{F}}(\Psi)\right] \sin \theta\left(\frac{\mathrm{d} \Psi}{\mathrm{d} \theta}\right) .
$$

Here, we have utilized definitions $(A .7)$ and $(A .8)$ and, for simplicity, written the equality $B_{\varphi}\left(r_{\mathrm{g}}\right)=-E_{\theta}\left(r_{\mathrm{g}}\right)$ for slow rotation. Thus, it is not surprising that in the framework of this approximation the mechanism of energy loss by a black hole was connected, in analogy with the unipolar inductor, with the Ampere force acting on the black hole horizon from the side of the surface current [131].

Only much later was it understood that the force-free approximation gives inaccurate and sometimes erroneous results. This is related to the fact that in the force-free approximation, i.e., when particles are assumed to be massless, the flow always remains subsonic. Under this assumption, the fast magnetosonic surface on which the 


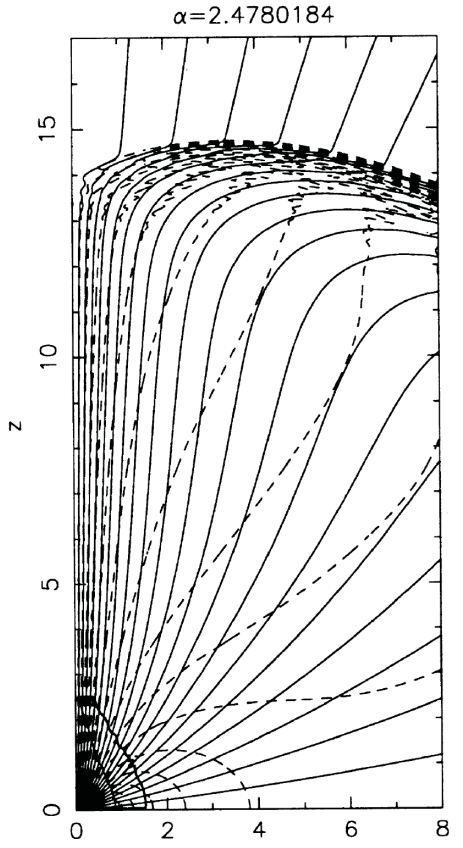

Figure 11: The structure of a magnetic field behind a switching-on wave propagating with velocity $c$ from a compact object [120]. Inside the switching-on wave, the flow rapidly becomes stationary, in correspondence with the analytical solution.

poloidal velocity of particles matches the fast magnetosonic wave velocity goes formally to infinity (and the inner surface, oppositely, coincides with the black hole horizon). But we have seen that it is the conditions on the fast magnetosonic surface that fix the longitudinal current circulating in the magnetosphere. In addition, the very necessity of establishing the boundary condition on the black hole horizon, i.e., in the causally disconnected region, shows that the physical interpretation given above does not relate to the reality [132].

To make this point more clear and, in particular, to understand the boundedness of stationary solutions (and hence the analytical method itself) in studies of the current closure, it is useful to consider the results obtained in paper [120] in which the problem was formulated as follows. There is a magnetized ball at rest which at the moment $t=0$ starts rotating with angular velocity $\Omega$. As a result, the switching-on wave starts propagating from the ball with velocity $c$, so that the magnetic field remains unperturbed beyond it and electric currents are absent, while inside the switching-on wave (and this is a very important result) the solution rapidly approaches the stationary transonic regime, which is in full agreement with the analytical solution. Thus, the assumption of the stationary solution for longitudal currents flowing actually along magnetic surfaces is confirmed.

As for the current closure, no current closure as such happens at all in the ideal case where the outflow oc- curs in a vacuum. This is related to the fact that in the switching-on wave the flow is time-dependent (Fig. 11), so there divj $\neq 0$ (S S Komissarov, private communication). In reality, the current closure will take place on a shock wave which must necessarily emerge in the region where the supersonic switching-on wave collides with the surrounding medium. In any case, however, the ambient medium for transonic flows cannot influence the magnitude of the longitudinal current for $r<r_{\mathrm{F}}$ and, hence, affect the central engine energy release. As soon as the switching-on wave crosses the singular surfaces (they are also shown in the left lower corner of Fig. 11), the longitudinal current flowing in the magnetosphere stops depending on time. For this reason, one can indeed consider in the framework of the stationary approximation that the electric current closure occurs at infinity, as is usually assumed. Thus, transonic flows are significantly different from subsonic ones, when the electric current circulating in the magnetosphere is determined by the conductivity of the boundary of the region occupied by plasma (see, for example, Ref. [133]).

Accounting for the nonzero mass of particles allows us to clarify the situation with the 'boundary condition on the horizon', and thus with the mechanism of energy release by a black hole. Indeed, as shown in Fig. 10, the fast magnetosonic surface for nonzero masses of particles is located above the black hole horizon. Therefore, the critical condition should also be set here, which is definitely located in the region casually connected to the outer space. The black hole horizon will be located in the region of the supersonic flow and, hence, cannot affect the properties of the flow. As a result, the additional critical condition must also be kept in the force-free limit $m_{\mathrm{p}} \rightarrow 0$, when the fast magnetosonic surface, as noted above, formally coincides with the event horizon. It is not then surprising that this limit of the critical condition on the fast magnetosonic surface exactly coincides with condition (74) [10]. Thus, the boundary condition on the horizon (74), which was necessarily used in the force-free approximation, represents a relict of the critical condition on the fast magnetosonic surface.

Correspondingly, it also becomes clear how to interpret the infinite time retardation near the black hole horizon. Indeed, the time it takes for the plasma to reach the horizon must be infinite from the point of view of the remote observer. As the time of existence of the black hole (the surrounding plasma, etc.) is finite, the remote observer will register a 'switching-on wave' corresponding to the very initial stages of the existence of the central engine over the black hole surface. This was, in fact, one more argument in support of the fact that it is incorrect to set any boundary condition on the black hole horizon [132]. However, as seen by the example of the outflow (see Fig. 11), to form the current system that fully determines the central engine power it is sufficient to wait a finite time until the plasma intersects the fast magnetosonic surface. This also implies, inter alia, that after a finite time the switching-on wave will turn out to be in the supersonic region of the flow and, hence, cannot affect the magnetosphere structure. This conclusion 


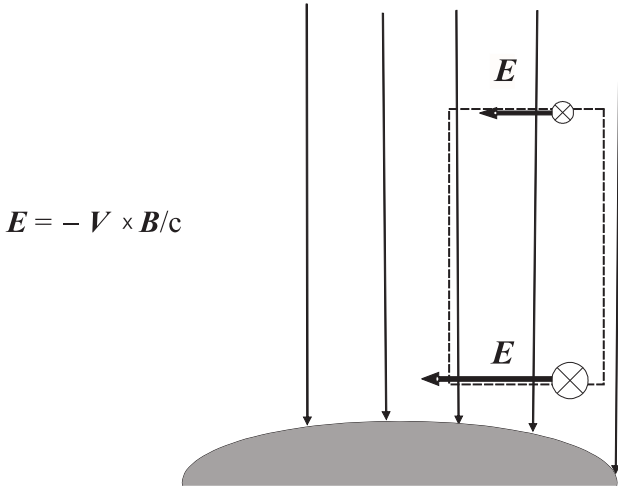

Figure 12: The appearance of the electromotive force in a circuit at rest relative to a rotating black hole immersed in an external magnetic field. The electric field directions are shown for observers at rest.

was numerically confirmed by Komissarov [134].

If this is indeed the case, however, another source of EMF should be found, since the black hole itself now can no longer be the source of extraneous forces and, consequently, serve as a battery. It turned out that the appearance of EMF in the black hole magnetosphere is related to the Lense-Thirring (the frame-dragging) effect, which appears due to rotation of the black hole. Indeed, according to general relativity, the space itself in the vicinity of a Kerr black hole starts rotating with angular velocity $\omega(A .4)$. Only in the reference frame rotating with this angular velocity will an observer not register a precession of gyroscopes. On the contrary, noninertial forces will appear in the laboratory frame at rest with respect to remote observers and they can be detected. It is the frame-dragging effect that leads to the appearance of the electromotive force in the black hole magnetosphere.

Indeed, as in the case of any body moving in the magnetic field, the 'motion of space' relative to the observer at rest will produce the electric field $\mathbf{E}=-\mathbf{V} \times \mathbf{B} / c$, where now $\mathbf{V}=\omega \times \mathbf{r}$ is the velocity of the body at rest relative to the preferred reference frame. Here, it is important that the angular velocity $\omega$ be different at various distances from the black hole. Therefore, as shown in Fig. 12, the circulation of the electric field in a circuit will be nonzero even if the electric circuit is at rest relative to the black hole, i.e., when the magnetic flux through the contour remains constant (of course, this state is possible only above the ergosphere). It is the motion of space through the circuit that generates the electromotive force. We see here that the 'electric battery' will be located above the black hole horizon, and possibly outside the ergosphere.

Finally, as already stressed, for positive energy flux from the central engine the electric currents in the black hole magnetosphere can flow only in the case where the plasma generation mechanism is operating above the horizon. Electron-positron pair production in the col- lision of hard gamma-ray quanta emitted from the accretion disc surface was already discussed above. Here, we should note that, as in the Penrose effect, one particle should fall into the black hole, and another particle should escape to infinity. It should be recalled that the Penrose effect has its origins in the remarkable property of rotating black holes - the relativistic mass defect can exceed $100 \%$ inside the ergosphere $r_{\mathrm{g}}<r<r_{\mathrm{e}}=M+\sqrt{M^{2}-a^{2} \cos ^{2} \theta}(G=c=1)$ and, hence, above all the horizon surface [14]. Therefore, it becomes evident that the Blandford-Znajek effect is in fact the electromagnetic realization of the Penrose process. The difference is that it concerns not charged particles themselves but the electromagnetic field they induce. In other words, the spin-down of the black hole is not due to electric currents flowing over the horizon but due to the negative electromagnetic energy flux falling onto the black hol. It is this role of the ergosphere inside which the relativistic energy of any material bodies (including the electromagnetic field) can become negative that is great. Such interpretation seems now to be the most likely, and most researchers involved in these studies tend to accept this interpretation (see, for example, book [135]).

Let us summarize. We have shown that the critical conditions on the fast magnetosonic surface mostly determine the energy release of the central engine. This surface serves as a valve that determines the magnitude of the longitudinal current circulating in the magnetosphere. Like the usual sonic surface in hydrodynamics, it separates subsonic and supersonic parts of the flow. As a result, the longitudinal current for transonic flows must be determined not by the external conditions but by the condition of smoothly crossing the singular surfaces. Even more complicated is the situation in a black hole magnetosphere, where both the current and the angular velocity $\Omega_{\mathrm{F}}$ should be deduced from the critical conditions on the singular surfaces. In consequence of this, it is these critical conditions on the singular surfaces that will determine the central engine power.

\section{Theoretical predictions}

\subsection{Collimation}

4.1.1 The force balance across the flow. Let now analyze the results of the analytical theory. First of all, we should try to formulate some general properties of a magnetized outflow, which must show their worth at large distances from the central object. As already noted, we are primarily interested in transonic flows, in which the flow at large distances is supersonic. Moreover, we shall unconditionally assume that the solution can be continued to infinity. This is possible, as we have seen, only if the longitudinal electric current is sufficiently large. As we have already stressed, thermal effects can almost always be neglected. As a consequence, Bernoulli equation becomes a fourth-order algebraic equation with respect to $\mathcal{M}^{2}$, which in many cases 


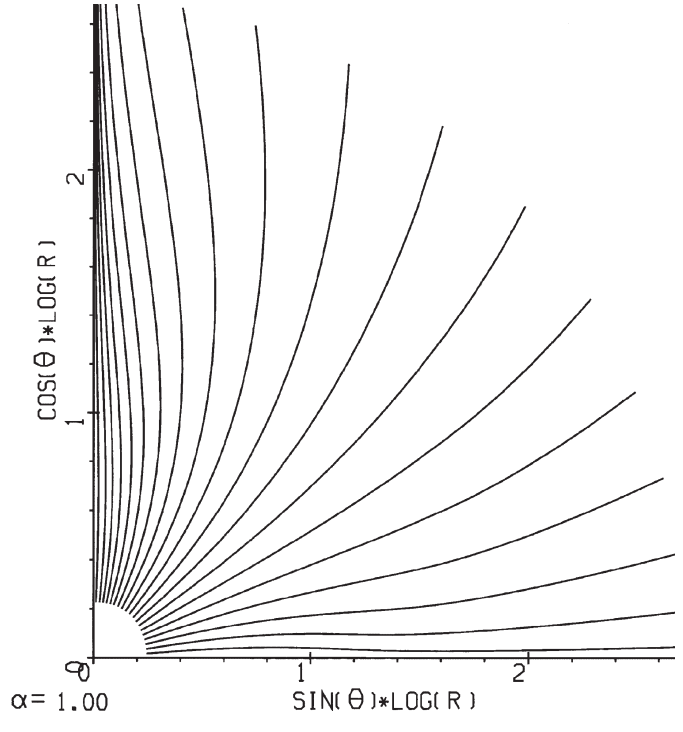

Figure 13: The structure of magnetic surfaces for a nonrelativistic plasma outflow in the split monopole magnetic field [120]. The decollimation in the region of the reverse bulk current near the central engine is clearly seen. However, the redistribution of longitudinal currents occurs at far distances, so that the current flowing out is concentrated near the rotational axis, and the reverse current flows near the equatorial plane.

allows us to write out rather simple analytical asymptotic solutions.

For simplicity, let us start from the nonrelativistic case. By analyzing the leading terms in the GradShafranov equation (41), it is possible to show that the force balance equation can be written in the form [136]

$$
\frac{\rho v_{\|}^{2}}{R_{\mathrm{c}}}=\frac{1}{c} j_{\|} B_{\varphi}
$$

where $R_{\mathrm{c}}$ is the radius of the magnetic field line in the poloidal plane. In other words, the equilibrium must be established due to the balance between the centrifugal force $\rho v_{\|}^{2} / R_{\mathrm{c}}$ and the Ampere force related to the longitudinal electric current $j_{\|}$. It is evident that the Ampere force, depending on the sign of the longitudinal current, can both collimate and decollimate the flow. In this case, the collimation must occur close to the jet axis, while the decollimation occurs at its periphery. Exactly this behavior was obtained both analytically [111] and numerically [120] (Fig. 13). It should be stressed that here we do not consider how strongly the magnetic surfaces can be bent and only investigate their form.

A similar picture, however, cannot be realized up to very large distances from the central engine. 'Natura abhorret vacuum', and the diverging magnetic surfaces inevitably must start collimating. It turned out that this is possible exactly because the longitudinal current $I$ is not the integral of motion, and hence the electric current, unlike particles, can intersect the magnetic surfaces. Indeed, it is easy to check that, for an almost radial flow at large distances, the right-hand side of equation (75) should decrease as $r^{-3}$, while the numerator on the lefthand side decreases as $r^{-2}$. As a result, for equality (75) to hold, the radius of curvature of the magnetic surfaces, $R_{\mathrm{c}}$, should increase as $r$. But such a behavior cannot be realized at mathematical infinity [137], so for $r \rightarrow \infty$ only the right-hand side of equation (75) is leading.

In the long run, we come at first glance across the paradoxical result that the current density in the magnetosphere must vanish at large distances [138]:

$$
j_{\|}=0
$$

In fact, this simply means that at large distances almost all outflowing longitudinal current must be concentrated near the rotational axis. Indeed, as shown in Ref. [139], a cylindrical region containing almost all outflowing current must inevitably appear near the axis 4 This behavior was later verified by numerical simulations [120]. We shall consider this point in more detail in Section 4.1.3.

In the relativistic case, the electric force $\rho_{\mathrm{e}} \mathbf{E}$ and the component of the Ampere force related to the longitudinal current $\mathbf{j}_{\|} \times \mathbf{B}_{\varphi}$ will mostly act on the outflowing plasma. However, as follows from equation (60), these forces almost mutually balance each other. Therefore, in addition to the bulk force 5

$$
\mathcal{F}_{\mathrm{jpol}} \approx \rho_{\mathrm{e}} \mathbf{E}-\nabla\left(\frac{B_{\varphi}^{2}}{8 \pi}\right),
$$

it is necessary to take into account the bulk centrifugal force

$$
\mathcal{F}_{\text {cent }} \approx \frac{n m c^{2} \gamma+S / c}{R_{\mathrm{c}}}
$$

( $S \approx c B_{\varphi}^{2} / 4 \pi$ is the Poynting vector) and the Ampere force $\mathcal{F}_{\text {jtor }} \approx \mathbf{j}_{\varphi} \times \mathbf{B}_{\mathrm{p}} / c$ pertaining to toroidal current. As a consequence, the Grad-Shafranov equation in the limit $r \gg r_{\mathrm{F}}$ can be conveniently rewritten in the form $[136,138,140]$

$$
\begin{array}{r}
\frac{B_{\varphi}^{2}+4 \pi n m_{\mathrm{p}} c^{2} \gamma}{R_{\mathrm{c}}}+\frac{1}{2} \hat{\mathbf{n}} \cdot \nabla\left(\mathbf{B}_{\mathrm{p}}^{2}\right) \\
+\frac{1}{2} \hat{\mathbf{n}} \cdot \nabla\left(B_{\varphi}^{2}-\mathbf{E}^{2}\right)-\frac{B_{\varphi}^{2}-\mathbf{E}^{2}}{\varpi}\left(\hat{\mathbf{n}} \cdot \mathbf{e}_{\varpi}\right)=0,
\end{array}
$$

where $\hat{\mathbf{n}}=\nabla \Psi /|\nabla \Psi|$. Notice that the Poynting vector contributes, in addition to the contribution from particles, to the centrifugal force. This is related to the fact

\footnotetext{
${ }^{4}$ Strictly speaking, this terminology corresponds to the case of $\mathbf{\Omega B}<0$, where $\rho_{\mathrm{GJ}}>0$. For the opposite orientation, the current near the rotational axis will flow towards the central engine.

${ }^{5}$ The corresponding formula (4.227) from monograph [9] has the incorrect sign
} 
that, as noted above, both particles and the electromagnetic energy propagate along the magnetic surfaces.

4.1.2 The collimation mechanism. Thus, the form of the magnetic surfaces close to the rotational axis depends on the balance between the collimating Ampere force arising from the longitudinal electric current (parallel currents are attracted) and the decollimating Ampere force related to toroidal currents. Thus, the question as to whether the collimation will be effective depends on the magnitude of the longitudinal current. A series of exact solutions [110, 111], which were possible to obtain by analyzing small deviations from the monopole magnetic field, showed that for nonrelativistic jets the collimation is large even close to the fast magnetosonic surface. This property is also confirmed by numerical modeling [120, 141] (see also Fig. 13).

In the relativistic case, where, as we remember, the longitudinal current is close to the Goldreich current $\left(i_{0} \approx 1\right)$, an almost full compensation of these two forces takes place. In particular, the balance is met exactly in the force-free approximation and in the split monopole magnetic field [142], so the vacuum monopole solution remains exact up to infinity for the magnetosphere filled with the plasma as well (Fig. 14). The current $I$ here takes the form $I(\theta)=I_{\mathrm{M}}^{(\mathrm{A})} \sin ^{2} \theta$, where

$$
I_{\mathrm{M}}^{(\mathrm{A})}=\frac{\Omega_{\mathrm{F}} \Psi_{0}}{4 \pi},
$$

which exactly coincides with the Goldreich current $\left(j_{\|}=\rho_{\mathrm{GJ}} c\right)$.

For massive particles (and again for the split monopole magnetic field), the longitudinal current determined from the condition of smoothly crossing the fast magnetosonic surface will differ from the Goldreich current only by a factor of the order $\sigma^{-4 / 3}[143]$. As a result, the perturbation of the magnetic flux function $\delta \Psi / \Psi$ in the asymptotically remote region $r \gg r_{\mathrm{F}}$ will increase logarithmically slowly $[143,144]$ :

$$
\frac{\delta \Psi}{\Psi} \sim \sigma^{-2 / 3} \ln ^{1 / 3}\left(\frac{r}{r_{\mathrm{F}}}\right) .
$$

In other words, the current turns out to be only insignificantly larger than the critical one, which leads to a vanishingly small collimation. Correspondingly, the particle energy also increases very slowly:

$$
\gamma \approx \sigma^{1 / 3} \ln ^{1 / 3}\left(\frac{r}{r_{\mathrm{F}}}\right) .
$$

We stress that above we have considered the proper collimation, i.e., that due to bulk currents. However, the collimation, generally speaking, can be produced in the source itself. In Fig. 15, the flow obtained as a small perturbation of the force-free solution is shown, but for a parabolic field [145]. Such a field may be generated in the accretion disc as well [5]. For not too small $\left(\theta \gg \gamma_{\text {in }}^{2} / \sigma\right)$ and not too large $\left(\theta \ll \sigma^{-1 / 3}\right)$ angles, the location of the

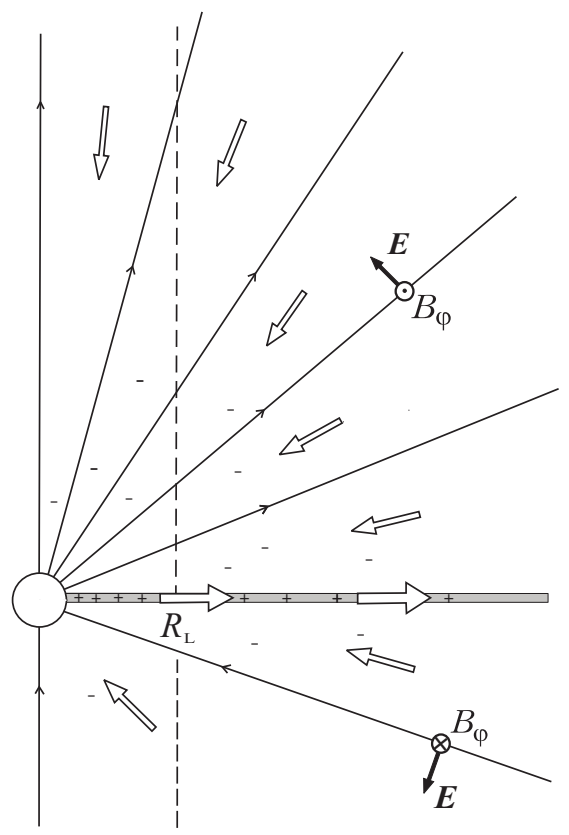

Figure 14: The force-free monopole solution found by Michel [142], in which the electric field $E_{\theta}$ exactly equals the toroidal magnetic field $B_{\varphi}$. The contour arrows show the direction of the poloidal current.

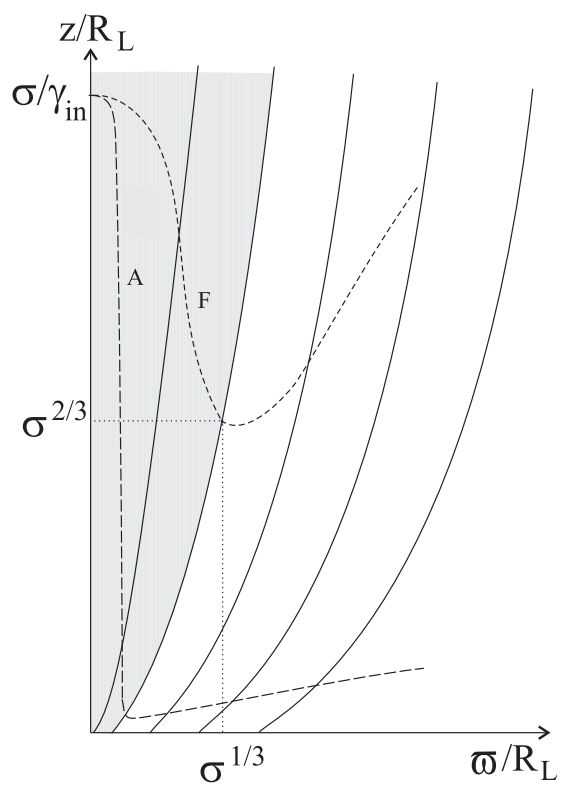

Figure 15: The location of the Alfvénic (A) and fast magnetosonic $(\mathrm{F})$ surfaces in a parabolic magnetic field. Such a field can also be generated in the accretion disc [145]. 
fast magnetosonic surface $r_{\mathrm{F}}$ and the value of $\gamma\left(r_{\mathrm{F}}\right)$ are given by the expressions

$$
\begin{aligned}
r_{\mathrm{F}} & \approx\left(\frac{\sigma}{\theta}\right)^{1 / 2} R_{\mathrm{L}}, \\
\gamma\left(r_{\mathrm{F}}\right) & \approx \sigma^{1 / 2} \theta^{1 / 2},
\end{aligned}
$$

respectively, which again correspond to the estimates of the particle energy, $\gamma \sim \sigma^{1 / 3}$, and the axial distance, $r_{\mathrm{F}} \sin \theta \sim \sigma^{1 / 3} R_{\mathrm{L}}$ obtained above (see Ref. [145] for more detail). But in this case the account for nonzero particle masses also only slightly perturbs the force-free solution. On the fast magnetosonic surface, for example, one finds

$$
\left(\frac{\delta \Psi}{\Psi}\right)_{r=r_{\mathrm{F}}} \approx \frac{1}{\sigma \theta} \ll 1 .
$$

Thus, we can conclude that the proper collimation is impossible in the relativistic case. That is, the bulk collimation due to longitudinal currents flowing in the magnetosphere is impossible. Therefore, the magnetic surfaces can be collimated either by a special choice of currents in the source itself, or due to the interaction of the supersonic wind with the surrounding medium. Clearly, a sufficiently extended dense disc is required in the first case. Because of this, for radio pulsars where there is definitely no such disc, one usually assumes that the flow at distances $r \gg R_{\mathrm{L}}$ must be radial.

Unfortunately, it is not clear at present which mechanism is actually responsible for the collimation of relativistic jets. However, if one assumes that the collimation is indeed caused by the presence of the surrounding medium, it becomes possible to estimate the transverse size $r_{\text {jet }}$ of the jets. Indeed, by assuming that the pressure of the poloidal magnetic field in the jet is close to the ambient pressure $P_{\text {ext }}$ (this estimate is valid by assuming the total electric current in the jet to be zero), we obtain from the condition of the magnetic flux conservation that

$$
r_{\mathrm{jet}} \sim R\left(\frac{B_{\mathrm{in}}^{2}}{8 \pi P_{\mathrm{ext}}}\right)^{1 / 4}
$$

where $R$ and $B_{\text {in }}$ are the radius and the magnetic field of the compact object, respectively. For example, for active galactic nuclei $\left(B_{\mathrm{in}} \sim 10^{4} \mathrm{G}, R \sim 10^{13} \mathrm{~cm}\right)$ we have

$$
r_{\mathrm{j}} \sim 1 \mathrm{pc},
$$

which exactly corresponds to the observed transverse sizes of jets [12]. Correspondingly, for young stellar objects $\left(B_{\text {in }} \sim 10^{3} \mathrm{G}, R \sim 10^{11} \mathrm{~cm}\right)$ we have $r_{\mathrm{j}} \sim 10^{16} \mathrm{~cm}$, which again is in agreement with observations. Thus, the external medium apparently must significantly affect the collimation of jets.

4.1.3 The dense core. As we have seen, near the axis the bulk force $\mathcal{F}_{\text {jpol }}$ related to the poloidal current is always directed toward the rotational axis, and in the region of the current closure, away from the axis. Then, for example, close to the cylindrical core where the curvature of the magnetic surfaces is small and, hence, the centrifugal force $\mathcal{F}_{\text {cent }}(78)$ can be neglected, the equilibrium can be established only if the force $\mathcal{F}_{\text {jtor }}$ is directed away from the rotational axis. But the poloidal magnetic field in this case must decrease with axial distance. Then, the density of the outflowing plasma will decrease along with the poloidal field as well. Exactly such a behavior of the solution was illustrated in Fig. 13.

It turned out that the dense core for the nonrelativistic supersonic flow will exist for both strongly and weakly magnetized flows close to the central engine, irrespective of the ambient pressure [146]. The radius of the core will correspond to such a distance from the axis, at which the toroidal field matches the poloidal one. A straightforward calculation shows that in both cases the longitudinal current in the core region will be $j=\left(c / v_{\text {in }}\right) j_{\mathrm{GJ}}$, i.e., $i_{0}=c / v_{\text {in }}$, and so one arrives at

$$
r_{\text {core }}=\frac{v_{\text {in }}}{\Omega} .
$$

The magnetic flux in such a core must be a significant fraction of the total magnetic flux. Such a configuration is none other than the $z$-pinch well-known in plasma physics [147] (the question of stability will be briefly discussed below).

As for the relativistic flow, the appearance of the dense core can be balanced here by the electric force $\rho_{\mathrm{e}} \mathbf{E}$ which, as we have seen, significantly weakens the force $\mathcal{F}_{\text {jpol }}$. As a result, the answer is significantly dependent on the ambient pressure [9]. If the relativistic jet is surrounded by a medium with the total gas and magnetic pressure $P_{\text {ext }}$ above some limiting value $P_{\min }$, the dense core does not form at all. In this case, the poloidal magnetic field in the jet will be approximately constant: $B_{\mathrm{p}}^{2} / 8 \pi \approx P_{\text {ext }}$. For convenience sake, we shall express below the limiting value $P_{\text {ext }}$ through the equivalent magnetic field $\left(B_{\min }^{2} / 8 \pi=P_{\min }\right)$. Here, one obtains the relationship

$$
B_{\min }=\frac{1}{\sigma \gamma_{\text {in }}} B\left(R_{\mathrm{L}}\right),
$$

where $B\left(R_{\mathrm{L}}\right)=\Omega^{2} \Psi_{\text {tot }} / \pi c^{2}$ is the characteristic magnetic field on the light cylinder. Correspondingly, the density of the outflowing plasma will be constant, too.

If the ambient pressure is sufficiently low, so that $P_{\text {ext }}<P_{\min }$, then, as in the nonrelativistic case, the dense core is formed in the center of the outflow; the radius of the core must exceed that of the light cylinder:

$$
r_{\text {core }}=\gamma_{\text {in }} R_{\mathrm{L}} .
$$

It is easy to check that on the core boundary the energy flux density of the electromagnetic field matches the particle energy density. The appearance of such a cylindrical jet was predicted already many years ago in many papers $[138,148,149]$, but the magnetic flux in this core was determined only quite recently $[145,146]$. The magnetic field near the axis was found to only very weakly (logarithmically) depend on the ambient pressure, so with 


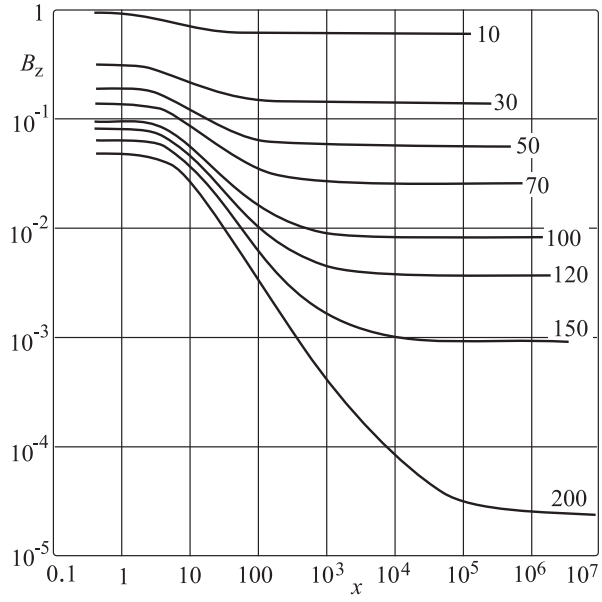

Figure 16: Longitudinal magnetic field $B_{z}(\mathrm{G})$ as a function of the axial distance $x=\Omega \varpi / c$ for $\sigma=10^{3}$ and different Mach numbers $\mathcal{M}$ on the jet axis, which was obtained as a solution of the one-dimensional problem [146]. The axial magnetic field only slightly deviates from $B_{\text {min }}$ when the ambient pressure changes by several orders of magnitude.

good accuracy one can set $B_{\text {core }}=B_{\min }$. As a result, the magnetic flux $\Psi_{\text {core }}=\pi r_{\text {core }}^{2} B_{\min }$ within the core turns out to be much smaller than the total flux:

$$
\frac{\Psi_{\text {core }}}{\Psi_{\text {tot }}} \approx \frac{\gamma_{\text {in }}}{\sigma} \ll 1 .
$$

Here, the poloidal magnetic field and the density of matter for $x_{r}=\varpi / r_{\text {core }}>1$ must behave as power functions

$$
\begin{gathered}
B_{\mathrm{p}} \propto x_{r}^{-k_{1}}, \\
\rho^{(\mathrm{lab})} \propto x_{r}^{-k_{2}} .
\end{gathered}
$$

respectively. As the ambient pressure decreases, the exponents $k_{1}$ and $k_{2}$ gradually increase; however, their difference remains approximately constant, viz.

$$
k_{1}-k_{2} \approx 0 .
$$

As a result, if we have $k_{1} \approx k_{2} \approx 0$ for $P_{\text {ext }}>P_{\text {min }}$ (i.e., the poloidal magnetic field and the outflowing plasma density are constant in the cross section), for ambient pressures corresponding to the magnetic field $B_{\text {ext }} \equiv B_{\text {eq }}$, where

$$
B_{\mathrm{eq}}=\frac{1}{\sigma^{2}} B\left(R_{\mathrm{L}}\right),
$$

we have, in contrast, $k_{1} \approx k_{2} \approx 1$. Under such ambient pressures, as we shall see, the contribution from particles to the energy flux becomes dominant in the entire volume of the jet.

In Fig. 16, the behavior of the poloidal magnetic field is shown in the double-logarithmic scale as a function of

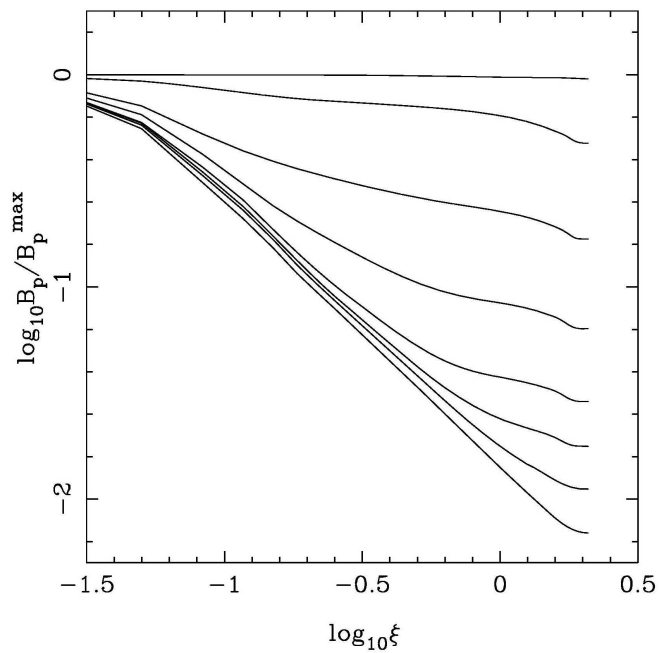

Figure 17: Longitudinal magnetic field profile as a function of the axial distance $\xi \propto \varpi$ for different values of the ambient pressure, which was obtained as a solution of the two-dimensional time relaxation problem [126]. The power-law dependence corresponds to the analytical estimate (92).

the distance to the axis [146] (see also Ref. [150]). It was obtained as the solution to the system of two ordinary differential equations $(A .14)$ and $(A .27)$ to which, as shown in the Appendix, the Grad-Shafranov equation can be conveniently reduced in the cylindrical case. The Mach number on the rotational axis served as the parameter for different curves. It is evident that the poloidal magnetic field at distances exceeding $r_{\text {core }}$ indeed starts decreasing as a power law. At the boundary of the jet it changes by several orders of magnitude, while the magnetic field on the axis changes only several-fold times. This behavior was recently confirmed by numerical two-dimensional calculations, too [126] (Fig. 17). It is important here that in the last case the very formulation of the problem was principally different (the time relaxation problem was solved rather than a stationary problem).

In relativistic jets there can be one more regime which is impossible in nonrelativistic jets [151]. In Fig. 15, it corresponds to cross sections located sufficiently close to the equator, when the flow in the jet center must be subsonic. By rewriting the condition $z<\left(\sigma / \gamma_{\text {in }}\right) R_{\mathrm{L}}$ in terms of an ambient pressure, we obtain the inequality $P_{\text {ext }}>B_{\mathrm{cr}}^{2} / 8 \pi$, where

$$
B_{\text {cr }}=\frac{\gamma_{\text {in }}}{\sigma} B\left(R_{\mathrm{L}}\right) .
$$

In this case, a subsonic flow region must inevitably be formed in the inner parts of the jet with $\varpi<r_{\mathrm{s}}$, where

$$
r_{\mathrm{s}} \approx \sigma\left[\frac{8 \pi P_{\mathrm{ext}}}{B\left(R_{\mathrm{L}}\right)^{2}}\right]^{1 / 2} R_{\mathrm{L}} .
$$


At last, the subsonic flow is established in the entire jet volume for higher ambient pressures corresponding to the magnetic field pressure on the fast magnetosonic surface.

4.1.4 The current closure. As we have understood, according to the most wide-spread point of view, it is the electric current flowing along collimated outflows, which is responsible for the main jet energy release. Here, the following theorem can be formulated.

A stationary cylindrical jet with finite magnetic flux $\Psi_{\text {tot }}$ can be formed either with the nonzero full electric current $I\left(\Psi_{\text {tot }}\right) \neq 0$ or in the presence of a surrounding medium with nonzero pressure.

At first glance, these two variants fully contradict each other. However, this is not actually the case. As we have seen, a cylindrical core (which contains a significant part of the outflowing current) must be formed near the jet axis both in the relativistic and nonrelativistic cases. If the compact object were solitary in the Universe, the reverse current would indeed return near the equatorial plane. The closing of the current itself would occur in the switching-on wave. Thus, if one ignores the current closure region and studies only the structure of the central region, it is indeed possible to assume that the current closure takes place at infinity.

In fact, when a jet is immersed into a medium with a finite pressure, the reverse current, as one usually assumes, must flow along the boundary of the cocoon that forms due to the interaction of the supersonic wind with the external medium. The boundaries of the corresponding cocoons can be well seen in Figs 1 and 4. It is the force balance at the cocoon boundary that allowed the magnetic field $B_{\min }$ close to the jet axis to be determined [145].

4.1.5 The stability. In conclusion, it is necessary to briefly consider the problem of the stability of jets. The nonrelativistic z-pinches observed in the laboratory are known to be strongly unstable with respect to constrictions and screw modes [147, 152,153]. Therefore, the problem of jet stability has been widely discussed in the literature [154-160]. As shown in Fig. 4, however, jets from young stars at large distances indeed are similar to a sequence of flying blobs rather than to a regular flow. Only at small distances can the flow be considered quite regular (see Fig. 5).

In recent years, special laboratory experiments have been carried out under conditions maximally similar to those in the nonrelativistic jets $[161,162]$. In particular, the plasma velocity was as high as $100-400 \mathrm{~km}$ $\mathrm{s}^{-1}$ (which is comparable to the plasma flow velocity in jets from young stars), and the total current was about 1 MA. In these experiments, a strong instability leading to rapid fragmentation of the flow into individual blobs was also observed.

Nevertheless, it is not obvious that the instability of laboratory pinches can be considered as an undisputable evidence for the immanent strong instability of astrophysical jets. The point is that astrophysical jets are always 'specially prepared', since they come out from the quasispherical subsonic flow region. As a result, the plasma density and the longitudinal magnetic field profiles near the jet base turn out to be close to the equilibrium ones. In laboratory experiments, in contrast, the initial plasma density is usually very different from the equilibrium value [153].

As for relativistic jets (which at large distances also frequently show an irregular structure), they proved to be more stable [163 165]. The recent numerical simulations [166] (where the jet was found to be stable after more than 1000 rotations of the central engine) confirmed this conclusion. Thus, there are no doubts now that the nonrelativistic Kruskal-Shafranov stability criterion [153]

$$
\frac{r_{\mathrm{jet}}}{L} \frac{B_{\mathrm{p}}}{B_{\varphi}}>1,
$$

where $L$ is the length of the jet, cannot be applied to relativistic flows. Unfortunately, the limits of the present review do not allow us to discuss this most important point in more detail.

\subsection{Acceleration}

4.2.1 The acceleration mechanism. First of all, let us consider the acceleration mechanism itself. It is convenient to start from expression (43) for the toroidal velocity of the non- relativistic flow. It is evident that in the subsonic region $\mathcal{M}^{2}<1$ the flow velocity corresponds to the precise corotation:

$$
v_{\varphi} \approx \Omega_{\mathrm{F}} \varpi .
$$

In other words, particles can be considered as beads on a wire that determines their angular velocity of rotation. This situation is quite understandable because, as can be easily checked, the energy density of the magnetic field within the Alfvénic surface exceeds the plasma energy density, so it is the magnetic field that controls the motion of particles. In fact, the magnetic field plays the role of a slingshot that provides the constant angular velocity of the plasma rotation. Therefore, the velocity of particles linearly increases with increasing axial distance. Then, the following theorem can be formulated.

In the nonrelativistic limit, the smooth crossing of the fast magnetosonic surface is possible only if the particle energy flux on this surface is at least one-third of the total energy losses. In other words, the transonic nonrelativistic flow in the supersonic region must already be effectively accelerated $(q \sim 1)$.

Indeed, we can set $E_{\mathrm{n}} \approx \Omega_{\mathrm{F}} I / 2 \pi c \eta_{\mathrm{n}}$ for strongly magnetized flows near the surface of the central engine, which immediately yields $E_{\mathrm{n}} \approx \Omega_{\mathrm{F}}^{2} r_{\mathrm{F}}^{2}$ for $I \approx i_{0} I_{\mathrm{GJ}}$. Moreover, it is easy to show that the poloidal velocity $v_{\mathrm{p}}$ near the singular surfaces also becomes on the order of $\Omega_{\mathrm{F}} r_{\mathrm{F}}$, too.

By this means the particle acceleration mechanism in the strongly magnetized wind is similar to that by which a slingshot accelerates a stone. This becomes possible exactly due to the dominant effect of the poloidal magnetic field. However, this acceleration stops at distances 
$r_{\mathrm{F}} \sim r_{\mathrm{A}}$, since there the magnetic field energy density becomes smaller than the plasma particle energy density. Additionally, the toroidal magnetic field beyond the Alfvénic surface becomes stronger than the poloidal one, so particles start sliding relative to the magnetic field lines. As a result, the flow passes to another asymptotic behaviour: $v_{\varphi} \approx \Omega_{\mathrm{F}} \varpi_{\mathrm{A}}^{2} / \varpi$.. The plasma kinetic energy in this region will be mainly due to their poloidal velocity component.

As for the particle acceleration in the ultrarelativistic limit, near the fast magnetosonic surface, as we have seen, the particle energy flux must be much smaller than the electromagnetic energy flux. So the question arises as to whether it is possible to effectively accelerate particles beyond the sonic surface. Surprisingly, the force balance across the flow should be considered once again to answer this question.

4.2.2 Efficiency. So, let us come back to equation (79). We have already mentioned that the particle energy will be completely determined by the drift motion beyond the light cylinder $\varpi \gg R_{\mathrm{L}}$. Therefore, using Eqn (59) we can write out the expression for the Lorentz factor of particles in the form

$$
\frac{1}{\gamma^{2}} \approx \frac{\mathbf{B}_{\mathrm{p}}^{2}}{B^{2}}+\frac{B_{\varphi}^{2}-\mathbf{E}^{2}}{B^{2}} .
$$

Now, making use of the relation $B_{\varphi} \approx|\mathbf{E}| \approx x_{r} B_{\mathrm{p}}$, where again $x_{r}=\Omega \varpi / c$, we immediately come to the conclusion that when the second term in expression (100) can be neglected, the particle Lorentz factor must approach the following asymptotic solution:

$$
\gamma=x_{r},
$$

which, as we shall see, is universal enough. If the curvature of the magnetic field lines is appreciable, then, in contrast, we can neglect in formula (79) the second term corresponding to the bulk force $j_{\varphi} B_{\mathrm{p}} / c$. As a result, by comparing the corresponding terms in the force balance equation (79) for the strongly magnetized (i.e., Poynting dominated) flow, we arrive at another asymptotic solution [167]:

$$
\gamma \approx \mathcal{C} \sqrt{\frac{R_{\mathrm{c}}}{\varpi}} .
$$

where $\mathcal{C} \sim 1$. Moreover, making use of Eqn (59) and the equilibrium condition (79), we can write in the general case the relationship [109]

$$
\frac{1}{\gamma^{2}} \approx \frac{1}{x_{r}^{2}}+\frac{\varpi}{\mathcal{C}^{2} R_{\mathrm{c}}}
$$

Here, the value of $\mathcal{C}$ can be exactly determined for strongly collimated flows and $\Omega_{\mathrm{F}}=$ const $[109,150]$ :

$$
\mathcal{C}=\sqrt{3} .
$$

Simply speaking, the Lorentz factor will be determined by the least of the two values giving by expressions (101) and (102).

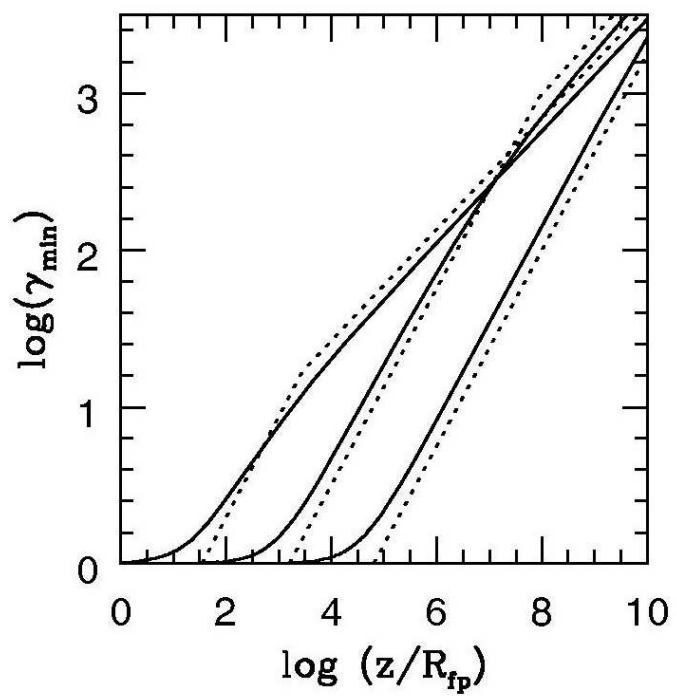

Figure 18: The growth of a particle's Lorentz factor $\gamma$ with distance from the equatorial plane $z$ [168]. The right curve corresponds to strong collimation $(k>2)$. For weaker collimation (left curves), the particle acceleration at large distances becomes less effective.

Thus, the choice between asymptotic solutions (101) and (102) must be determined by how bent the magnetic surfaces are. It is easy to show that the parabolic magnetic field in which the field line at a large distance from the central source is given by the equation $z(\varpi) \propto \varpi^{2}$ corresponds to the terminating case $[126,146]$. Indeed, as the curvature radius can be defined as $R_{\mathrm{c}}=\left[\left(z^{\prime}\right)^{2}+1\right]^{3 / 2} / z^{\prime \prime}$, in the limit $z^{\prime}=\mathrm{d} z / \mathrm{d} \varpi \gg 1$ for the magnetic surfaces specified by the relationship $z(\varpi) \propto \varpi^{k}$, Eqn (102) gives the energy of particles moving along the magnetic field line:

$$
\gamma \propto \varpi^{k-1}
$$

where $\varpi$ is, in this case, the current axial distance of a particle. At $k=2$, the acceleration efficiency determined from expressions (101) and (102) is the same. Thus, if the magnetic surfaces are collimated more strongly than those for the parabolic field (i.e., $k>2$ ), the curvature of the magnetic surfaces can be neglected at large distances, and the energy of the particles will be determined by expression (101). If the flow is poorly collimated (i.e., $1<k<2$ ), the particle acceleration will be less effective and one should use expression (102). The numerical simulations $[124,168]$ fully confirm the picture presented here. As shown in Fig. 18, the acceleration of a particle moving along the magnetic field line indeed follows the law $\gamma(z) \propto z^{1 / k}$ for strongly collimated flows, in correspondence with asymptotic behaviour (101), while the acceleration for poorly collimated outflows at large distances, in full agreement with expression (105), becomes less effective. 
We thus arrive at the most important conclusion that the acceleration efficiency of particles in a supersonic ultrarelativistic wind is determined by the degree of collimation of the magnetic surfaces. The plasma can be effectively accelerated only in the case where the magnetic surfaces are collimated more strongly than those for the parabolic field. In this event, one can use asymptotic solution (101) which shows that the acceleration mechanism, in fact, again is due to the slingshot effect (the larger the axial distance, the higher the energy). But in this case, too, the total transformation of the electromagnetic energy into the particle flux energy, $\gamma \approx \sigma$, can take place only if the jet transverse size exceeds the value

$$
r_{\mathrm{eff}}=\sigma R_{\mathrm{L}} .
$$

In particular, the fraction of energy carried by particles for $P_{\text {ext }}<B_{\text {eq }}^{2} / 8 \pi$ can be determined from the simple relationship [10,151]

$$
\frac{W_{\text {part }}}{W_{\text {tot }}} \sim \frac{1}{\sigma}\left[\frac{B^{2}\left(R_{\mathrm{L}}\right)}{8 \pi P_{\text {ext }}}\right]^{1 / 4} .
$$

If the collimation is poor, the acceleration will be ineffective, since particles begin sliding from the magnetic field lines. In this case, a much higher transverse size of the jet is required for accelerating particles to limiting energies:

$$
r_{\mathrm{eff}}=\sigma^{1 /(k-1)} R_{\mathrm{L}} .
$$

This dependence was also numerically confirmed [169]. In particular, the acceleration becomes actually impossible for the split monopole magnetic field $k=1$ (which, as we see, is a special case that requires separate consideration), since, as follows from estimate (82), the condition $\gamma \approx \sigma$ is reached only at exponentially large distances from the compact object. On the other hand, it easy to check that for $k>1$ both for effective and for ineffective acceleration mechanisms the condition $\gamma \vartheta \sim 1$ will be satisfied, where $\vartheta \approx \varpi / z$ is the characteristic opening angle of the acceleration region.

Here, it is important to emphasize that the selfconsistent analysis, in which the magnetic surfaces for the split monopole field were not assumed to be exactly conical, allowed the determination of the correct structure of the flow [143]. For example, the expression (82) for the particle's Lorentz factor discussed above is determined precisely by the small curvature of the magnetic surfaces, and so exactly corresponds to expression (102). It should be remembered that for a long time the flow in the split monopole magnetic field had been, in fact, the only example in which analytical results could be obtained. Therefore, a strong opinion was that the effective particle acceleration beyond the fast magnetosonic surface is completely impossible. As wee see, this conclusion proved to be incorrect in the general case.

\subsection{Subsonic flows}

As discussed above, all observed jets must be transonic. It is this property that allowed us to find the longitu-

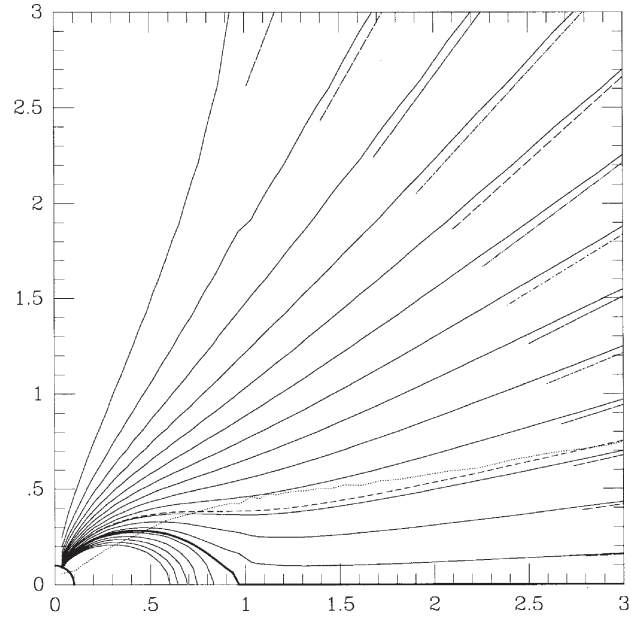

Figure 19: The structure of the axisymmetric radiopulsar magnetosphere in the model [98]. The flow is assumed to be radial at large distances.

dinal current flowing in the magnetosphere and, hence, to estimate the central engine power. However, until recently many models considered subsonic flows, and the magnitude of the current was determined from other considerations $[170,171]$. We shall briefly discuss below two the most known examples of such subsonic flows.

4.3.1 Pulsar magnetospheres. Starting in the early 1970s, pulsar magnetospheres have been discussed mostly in the force-free approximation [107, 142, 172174]. This was based on the fact that the plasma filling the neutron star magnetosphere is secondary with respect to the magnetic field, and so (at least inside the light cylinder) the particle energy density can be neglected. The Grad-Shafranov equation (A.28) in the force-free approximation (which in this case is simply called the pulsar equation) becomes elliptical. Therefore, for numerical modeling of the axisymmetric magnetospheres one has to impose an additional condition at the external boundary of the integration region [98, 100 104]. Usually, one chooses the condition of radiality of the magnetic field lines (Fig. 19). In this case it is this additional condition that fixes the longitudinal current in the magnetosphere. Thus, it is not surprising that this current turns out to be close to the longitudinal current $I_{\mathrm{M}}(\theta)$ (80) obtained by F C Michel for the monopole solution shown in Fig. 14. If the absence of the reverse current flowing along the equator (so that the current closing was done by bulk currents only) was chosen as the additional condition, the magnetic field structure beyond the light cylinder turned out to be significantly different [175].

Interestingly, a similar structure also appears in different models of the inclined rotator. Here, first of all, we should highlight the model of the 'rotating split monopole' (Fig. 20). In the force-free approximation 


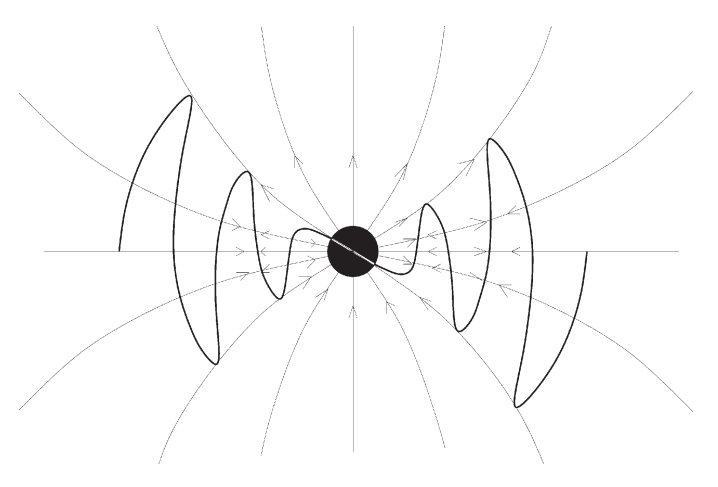

Figure 20: The magnetic field structure in the 'rotating split monopole' model [176]. In the force-free case, particles moving radially at the speed of light can provide the formation of the current sheet (the wavy curve) which separates magnetic fluxes in the equatorial region.

(when massless particles move with the speed of light), the monopole magnetic field, namely

$$
\begin{aligned}
& \Psi(r, \theta, \varphi, t)=\Psi_{0}(1-\cos \theta) \\
& \theta<\pi / 2-\chi \cos (\varphi-\Omega t+\Omega r / c) \\
& \Psi(r, \theta, \varphi, t)=\Psi_{0}(1+\cos \theta) \\
& \theta>\pi / 2-\chi \cos (\varphi-\Omega t+\Omega r / c)
\end{aligned}
$$

was found to be also the solution of the problem [176]. In this case, the electromagnetic fields inside cones with angles $\theta<\pi / 2-\chi$ and $\pi-\theta<\pi / 2-\chi$ near the rotational axis are independent of time and the angle $\varphi$ and coincide with the fields of an axisymmetric rotator, while in the equatorial plane the field directions reverse sign at the moment when the current sheet intersects the given point. A similar structure beyond the light cylinder was obtained for the rotating dipole, too [177]. True enough, in contrast to the 'rotating magnetic monopole', the total longitudinal current here depends on the inclination angle $\chi$, although not very strongly.

The last property can be easily explained. As we have seen, the very existence of the MHD wind far away from the light cylinder is possible only if the toroidal magnetic field is comparable in magnitude to the electric field. But this is possible only if a sufficiently large longitudinal current $I \approx I_{\mathrm{M}}^{(\mathrm{A})}$ flows in the magnetosphere. Let us keep in mind that this value of the current can also be found from the condition of the smooth crossing of the singular surfaces in the full MHD version. In none of the numerical calculations mentioned above were bounds on the longitudinal currents flowing from the neutron star surface set. Thus, it is not surprising that the longitudinal current obtained from the solution of the problem considered turned out to be on the order of $I_{\mathrm{M}}(\theta)$. As a result, the energy losses in model [176] were found to be independent of the angle $\chi$. Energy losses in model [177] were also found to be weakly dependent on the in- clination angle:

$$
W_{\mathrm{tot}} \approx \frac{1}{4} \frac{B_{0}^{2} \Omega^{4} R^{6}}{c^{3}}\left(1+\sin ^{2} \chi\right) .
$$

However, the following problem emerges here. All the theories of particle generation near the neutron star magnetic poles [178-180] unambiguously suggests that the longitudinal current density cannot be higher than that of the local Goldreich current which, as follows from definition (9), depends on the inclination angle $\chi$

$$
j_{\mathrm{GJ}} \approx \frac{\Omega B}{2 \pi} \cos \chi
$$

Indeed, the local Goldreich charge density near the magnetic poles for the orthogonal rotator must be $(\Omega R / c)^{1 / 2}$ times smaller than in the axisymmetric magnetosphere. Thus, the longitudinal current flowing along open magnetic field lines should be correspondingly smaller (for ordinary pulsars this factor can be as high as $10^{2}$ ). In the 'rotating split monopole' model considered above, this problem does not arise, because at any inclination angle in the polar magnetospheric regions the current is always the same as in the axisymmetric case. Just this current provided the necessary toroidal magnetic field. For the inclined dipole, in contrast, it is necessary to additionally assume that the longitudinal current in the polar cap regions can be significantly higher than the local Goldreich current (A Spitkovsky, private communication).

There is one more problem related to the decrease in the longitudinal current density as $\chi \rightarrow 90^{\circ}$. The point is that the current losses for the local Goldreich current must decrease as the inclination angle $\chi$ increases $[56,107]$ :

$$
W_{\text {tot }}=\frac{f_{*}^{2}}{4} \frac{B_{0}^{2} \Omega^{4} R^{6}}{c^{3}} i_{0} \cos \chi .
$$

Here, the coefficient $f_{*}=1.59-1.96$ depends only on the inclination angle $\chi$. It is necessary to stress that in addition to the $\cos \chi$ factor (which is related to the scalar product $W_{\text {tot }}=-\boldsymbol{\Omega} \cdot \mathbf{K}$, where $\mathbf{K}$ is the braking torque), the significant dependence of the current losses $W_{\text {tot }}$ on the inclination angle is also contained in the quantity $i_{0}$. The matter is that in the definition of the dimensionless current $i_{0}=I / I_{\mathrm{GJ}}$ in expression (113), the denominator contains the Goldreich current for the axisymmetric case, whereas at nonzero angles $\chi$ the Goldreich charge density itself depends on the angle $\chi$ near the magnetic poles. It is logical to expect that the dimensionless current $i_{0}$ for the inclined rotator will be bounded from above as $i_{0}^{(\max )}(\chi) \sim \cos \chi$. As a result, the current losses, in contrast to relationship (111), must decrease with angle $\chi$ at least as $\cos ^{2} \chi$. In particular, for $\chi=90^{\circ}$ (when $\cos ^{2} \chi$ must be replaced by its characteristic value within the polar cap region $\cos ^{2} \chi$, we obtain [56]

$$
W_{\mathrm{tot}}=c_{\perp} \frac{B_{0}^{2} \Omega^{4} R^{6}}{c^{3}}\left(\frac{\Omega R}{c}\right) i_{\mathrm{A}} .
$$




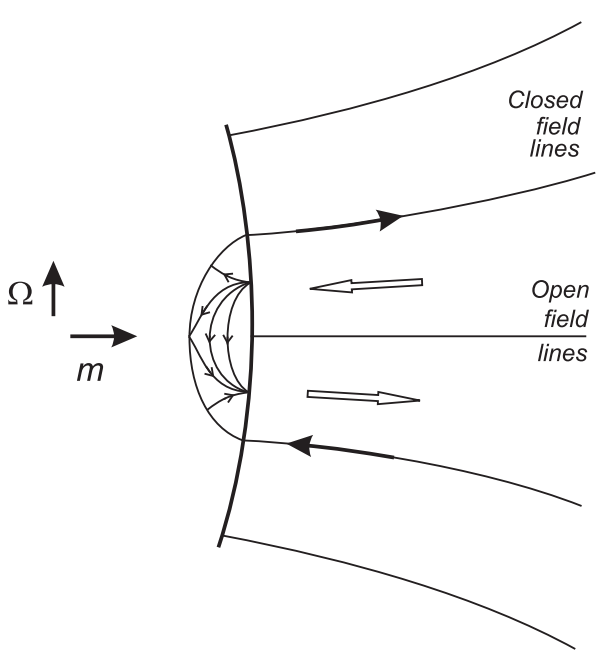

Figure 21: The structure of electric currents flowing near the magnetic poles of an orthogonal rotator. Currents flowing along the sepratrix (thin arrows) that separates the regions of the open and closed magnetic field lines are adjusted to bulk currents (contour arrows) in such a way that the closing surface current is fully concentrated within the polar cap.

Here, $i_{\mathrm{A}}=1$ for the local Goldreich current, and the coefficient $c_{\perp} \sim 1$ now depends not only on the profile of the asymmetric longitudinal current, but also on the polar cap shape.

Usually, when discussing this point, the following counter-argument against the decrease in current losses with increasing $\chi$ is invoked. In the expression for the braking torque, namely

$$
\mathbf{K}=\frac{1}{c} \int\left[\mathbf{r} \times\left[\mathbf{J}_{\mathrm{s}} \times \mathbf{B}\right]\right] \mathrm{d} S,
$$

The surface current $\mathbf{J}_{\mathrm{s}}$ indeed must decrease as $\cos \chi$ as angle $\chi$ increases. But then the characteristic distance from the axis to the polar cap, in contrast, will increase as $\sin \chi$, so ultimately the losses will be weakly dependent on the inclination angle $\chi$ even for the local Goldreich current.

As follows from a more precise analysis [107], however, the real structure of the surface currents in the polar cap region was ignored in this obvious, at first glance, consideration. Referring to Fig. 21, the closing currents in fact must be such that the current averaged over the polar cap region is zero. As a result, one needs to consider higher-order effects (like, for example, the neutron star surface curvature effect), when determining the radio pulsar spin-down rate. But if the surface current averaged over the polar cap region is indeed zero, then, as shown in Fig. 21, a surface current comparable in amplitude to the bulk current in the magnetosphere must flow along the separatrix separating the regions of open and closed magnetic field lines. Remarkably, in numerical modeling of the inclined rotator [181], reverse currents flowing along the separatrix were indeed discovered. Finally, it worth noting that there is no contradiction between relations (111) and (113), either. As we specially emphasized, the approximation expression (111) was obtained in Ref. [177] for a flow in which the longitudinal current was larger than the local Goldreich current, which corresponds to the condition $i_{0}>1$ ( $i_{\mathrm{A}}>1$, respectively).

In any case, studies of the last decade, in our opinion, have at last formulated the problem whose solution will provide significant progress in the understanding of the structure of radio pulsar magnetospheres. The problem is whether the plasma generation region in the neutron star magnetosphere can provide a sufficiently large longitudinal current which is necessary to launch the MHD wind from the inclined rotator. If the necessary current can be produced, nothing will prohibit the formation of the MHD wind in which the main part of the energy will be carried by the electromagnetic field. If the generation of a current which is significantly larger than the local Goldreich current is impossible, then the toroidal magnetic field near the light cylinder will be smaller than the poloidal magnetic field. In that case, a light surface will inevitably be formed near the light cylinder, on which the current closing and particle acceleration up to energies $\gamma \sim \sigma$ will occur [56]. Thus, the problem of the effective particle acceleration in pulsar winds, which we mentioned in Section 2.4, can be solved.

Interestingly, the possibility of answering this question has apparently emerged a short time ago. This test is related to the unusual properties of the radio pulsar PSR $B 1931+24$ [182]. It differs from other pulsars in that it stays in the active state for 5-10 days, and then its radio emission switches off in less than $10 \mathrm{~s}$, and the source is not observed during the next 25-35 days. It is important that the spin-down rate in these two states be different:

$$
\begin{aligned}
& \dot{\Omega}_{\mathrm{on}}=-1.02 \times 10^{-14} \mathrm{~s}^{-2}, \\
& \dot{\Omega}_{\mathrm{off}}=-0.68 \times 10^{-14} \mathrm{~s}^{-2},
\end{aligned}
$$

so that

$$
\frac{\dot{\Omega}_{\mathrm{on}}}{\dot{\Omega}_{\mathrm{off}}} \approx 1.5 .
$$

A similar behavior was later observed in the pulsar PSR $J 1832+0031\left(t_{\text {on }} \sim 300\right.$ days, $t_{\text {off }} \sim 700$ days $)$, with the ratio $\dot{\Omega}_{\text {on }} / \dot{\Omega}_{\text {off }} \approx 1.5$ again.

It is logical to assume that the difference in the spindown rates in these pulsars is simply associated with the fact that the spin-down in the switch-on state is due to the current losses, and in the switch-off state, when the magnetosphere is not filled with plasma, is due to magnetodipole radiation $[183,184]$. Then, making use of equations (3) and (113) we obtain

$$
\frac{\dot{\Omega}_{\mathrm{on}}}{\dot{\Omega}_{\mathrm{off}}}=\frac{3 f_{*}^{2}}{2} \cot ^{2} \chi,
$$

which yields the reasonable inclination angle $\chi \approx 60^{\circ}$. On the other hand, using expression (111) [177] for the 
switch-on state, we arrive at the relationship

$$
\frac{\dot{\Omega}_{\mathrm{on}}}{\dot{\Omega}_{\mathrm{off}}}=\frac{3}{2} \frac{\left(1+\sin ^{2} \chi\right)}{\sin ^{2} \chi} .
$$

Clearly, this quantity cannot be equal to 1.5 for any $\chi$. If this interpretation of observations holds true, this implies that the longitudinal current in the magnetosphere indeed does not exceed the local Goldreich current.

It should be emphasized that we have assumed above that the magnetospheric plasma fully screens the magnetodipole radiation of the neutron star. This conclusion, which was formulated for the first time in paper [107], seems now to be directly confirmed, since both in the model [176] and in the numerical calculations [107] there are no alternating electromagnetic fields decaying as $1 / r$.

4.3.2 'The magnetic tower'. 'The magnetic tower' is the model of collimated jets proposed by D LyndenBell in 1996 [128] (Fig. 22) and later on widely discussed in relation to both relativistic and nonrelativistic sources [171, 185-187]. It is based on the assumption that there exists an intensive wind outflowing perpendicular to the accretion disc and stretching the magnetic field lines along the rotational axis. Here, one usually assumes that the initial magnetic field was quasi-dipole, i.e., it consisted of magnetic field lines with one end frozen in the central star (or in the inner regions of the accretion disc) and another end frozen in the outer parts of the disc. Such a cylindric flow cannot intersect singular surfaces since the magnetic field lines remain at a constant distance from the axis of rotation. That is why, the longitudinal current in this model will be determined exactly by the differential rotation that leads to the magnetic field line twisting. Because of this, as in the case shown in Fig. 11, the magnetic tower top will propagate upwards by gradually increasing the volume occupied by the twisted magnetic field, while a stationary configuration restricted by the ambient pressure will be formed at smaller distances.

There are two additional important properties that distinguish the magnetic tower model from the transonic flows we are considering. First, if the magnetic field lines are anchored in the accretion disc, a configuration with almost zero total magnetic flux will form during the outflow (see Fig. 22). In other words, the direction of the poloidal magnetic field on the periphery of the jet will be different from that near the jet axis. Second, when the magnetic field loops do not extend beyond the light cylinder and, hence, do not open, the energy will continue being transferred along the magnetic fields lines. But this means that the energy will be carried away from the central engine only along the rotational axis, while on the jet periphery the energy flux will be directed back to the accretion disc $[186,187]$.

If such a configuration were stationary, the reverse energy flux would be exactly the same as the energy flux outgoing from the central engine closely to the rotational axis. However, we have seen that the equipotenital con-

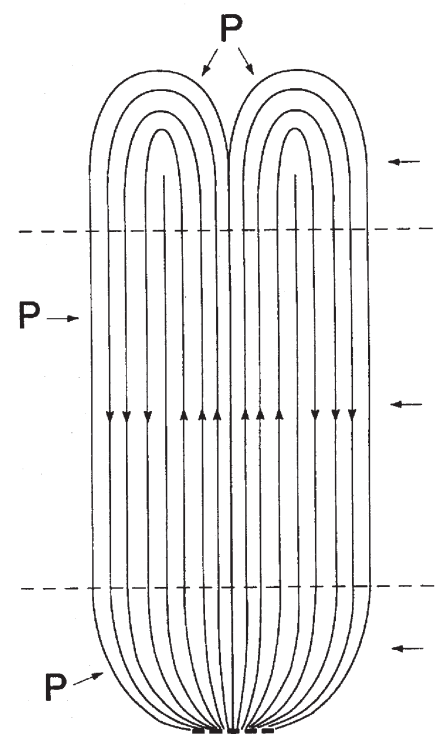

Figure 22: The magnetic field structure in the model of the 'magnetic tower' that can exist under sufficiently strong ambient pressure $P$ [128]. The energy is transferred from the central engine along the rotational axis, and back to the accretion disc along the jet periphery.

dition is violated in the switching-on wave, so the reverse energy flux turns to be smaller than the escaping flux.

Thus, in the very setup the problem of the magnetic tower formation is different from that describing transonic flows. The longitudinal current determining the energy losses in no way relates to the critical conditions on the singular surfaces which, as we specially stressed above, should unavoidably appear in all real compact sources (see also Section 2). Hence, apparently, the magnetic tower model cannot correspond to the reality. The results of numerical simulations also support this conclusion. When the flow intersected the singular surfaces, the magnetic field lines became open and the energy flux was directed outward from the central engine in the regions of both outgoing and incoming magnetic field lines [188]. On the other hand, in the case where the flow remained subsonic in numerical simulations, the magnetic tower formation was indeed observed $[50,189]$.

\section{Estimation of parameters}

Thus, we have seen that at present it has turned out to be possible to understand many key points related to the formation and the internal structure of collimated outflows. In doing so, we have managed to find several key parameters that determine the basic physical properties of ejected matter. First and foremost, these include the magnetization parameter $\sigma$, the multiplicity parameter $\lambda$, and the initial velocity $v_{\text {in }}$ (the Lorentz factor $\gamma_{\text {in }}$ ) of the outflow. We shall try below to understand how 
precisely these parameters can be estimated for the observed jets.

It should be emphasized from the very beginning that each time it is necessary to clearly separate which collimated outflows are being considered. Indeed, the properties of jets from active galactic nuclei can be quite different on the scale of the host galaxy (see Fig. 1) and in the formation region (see Fig. 2), where their transverse size is close to $1 \mathrm{pc}$. However, as we mentioned above, the relativistic jets can be stable on all scales. On the other hand, in the majority of cases nonrelativistic jets at large distances from the star can indeed be unstable (see Fig. 4), so for them the treatment considered here, strictly speaking, can be applied only in the innermost parts.

\subsection{Active galactic nuclei}

Despite longstanding efforts, we know very little about the internal structure of jets from active galactic nuclei. In particular, we still do not have an answer to the key question of whether it is the black hole, and not the inner parts of the accretion disc, that is the central engine which is responsible for the black hole power [190,191]. In the practical sense, the main uncertainty appears in the determination of the particle production multiplicity $\lambda$. Indeed, as already noted, the plasma on the magnetic field lines threading the black hole horizon (which is needed both to screen the longitudinal electric field and to produce the longitudinal electric current) must be generated in the magnetosphere itself between two families of singular surfaces. Some fraction of the plasma will escape the magnetosphere, while another part will accrete onto the black hole. Correspondingly, it is still unclear which quantities determine the density of matter flowing out of the accretion disc surface (see, for example, Refs [192, 193]). Moreover, it cannot be ruled out that the jet at large distances from the central engine will be additionally 'loaded' due to interaction with stellar winds from surrounding stars [194], or, for example, due to the 'photon breeding' effect (creation of the secondary electron-positron plasma by hard gamma-ray quanta generated in the interaction of the relativistic outflow with the ambient medium) $[195,196]$. That is why, the properties of jets on the scales of several kiloparsecs can be significantly different from those in the jet formation region.

In the region of field lines threading the black hole horizon several plasma generation mechanisms are currently being discussed, in which, however, plasma is ultimately generated always due to two-photon pair creation. The one-photon conversion, which plays the leading role in radio pulsar magnetospheres, here turns out to be ineffective, since the probability of pair creation in magnetic fields $B \sim B_{\text {Edd }} \sim 10^{4} \mathrm{G}$ is vanishingly small.

First and foremost, secondary plasma generation can be related to the direct two-photon process $\gamma+\gamma \rightarrow$ $e^{+}+e^{-}$(see, for example, Ref. [105]), where the necessary gamma-quanta are emitted from the inner regions of an accretion disc. This mechanism, with a high value of the parameter $\lambda_{\mathrm{AGN}_{1}} \sim 10^{10}-10^{12}$, was discussed in the pioneering paper by Blandford and Znajek [33]. However, sufficiently high temperatures providing the necessary number of hard gamma-ray photons with energies above the pair creation threshold $\mathcal{E}_{\text {min }}=m_{\mathrm{e}} c^{2}$, and small free path lengths of photons are required for this mechanism to be effective. Presently, the accuracy of the compactness parameter estimate $l_{a, \mathrm{AGN}} \sim 1-100$ does not allow one to make definitive conclusions on the efficiency of this mechanism of particle creation.

On the other hand, such a high particle density must be typical for a wind outflowing from the accretion disc surface. Let us keep in mind that even if the energy release related to such a wind is insignificant, it can play the decisive role in the matter outflow collimation [197]. Here, the energy of the jet core observed at high radio frequencies and in gamma-rays will be associated with ultrarelativistic particles extracting energy from the rotating black hole.

There is another mechanism capable of bringing particles into the region of magnetic field lines threading the black hole horizon even in the absence of hard gammaray quanta. This mechanism is similar to the particle creation process in the outer gap of the pulsar magnetosphere [198]. Indeed, the exact relativistic expression for the Goldreich charge density $\rho_{\mathrm{GJ}}$ takes the form [9]

$$
\rho_{\mathrm{GJ}}=-\frac{1}{8 \pi^{2}} \nabla_{k}\left(\frac{\Omega_{\mathrm{F}}-\omega}{\alpha} \nabla^{k} \Psi\right) .
$$

In particular, near the rotational axis we simply have

$$
\rho_{\mathrm{GJ}} \approx-\frac{\left(\Omega_{\mathrm{F}}-\omega\right) B}{2 \pi \alpha} .
$$

As a result, the general relativity effects cause the Goldreich density to vanish at $\omega \approx \Omega_{\mathrm{F}}$. Therefore, a region quite similar to the outer gap in pulsar magnetospheres appears in the black hole magnetospheres. The formation of longitudinal electric fields is also possible in this region, since the charge-separated plasma flow cannot provide the fullfilment of the condition $\rho_{\mathrm{e}}=\rho_{\mathrm{GJ}}$. As a result, it turned out that under real conditions the size of the acceleration region is much smaller than the system's size, so that the acceleration region does not affect the global structure of the magnetosphere [34, 35]. In this model, the particle production multiplicity is rather small:

$$
\lambda_{\mathrm{AGN}_{2}} \sim 10-100
$$

Hence, we shall consider below both large and small values of the parameter $\lambda$.

Next, it should be remembered that to explain the high efficiency of the energy release from the central engine we need to assume that the rotation parameter $\Omega R / c$ must be not too much smaller than unity. In other words, the light cylinder radius must not exceed the central engine size too much. As a result, the observed transverse size $r_{\text {jet }}$ of relativistic jets will be three-five orders of magnitude greater than the light cylinder radius $R_{\mathrm{L}}$. That is why, far from the central engine most 
magnetic field lines must be far beyond the light cylinder.

However, according to the relationship

$$
\frac{B_{\varphi}}{B_{\mathrm{p}}}=x_{r},
$$

where again $x_{r}=\Omega_{\mathrm{F}} \varpi / c$, which follows from the definition of the magnetic field components at $I \approx I_{\mathrm{GJ}}$, this implies that the toroidal magnetic field will be the same three-five orders of magnitude stronger than the poloidal magnetic field. Therefore, the magnetic field must have a strongly pronounced spiral structure. Correspondingly, the electric field must also be three-five orders of magnitude larger than the poloidal magnetic field. At present, VLBI (Very Large Baseline Interferometry) methods provide a lot of data on the polarization of the innermost parts of jets [199 201]; however, so far it is impossible to unambiguously determine the magnetic field structure from the observations.

Notice, finally, that strong twisting of magnetic field lines does not imply that the plasma motion will also occur along strongly twisted trajectories. As stressed above, an almost poloidal drift motion in the crossed electric and magnetic fields will be the principal motion of particles beyond the light cylinder. Thus, the toroidal velocity for $x_{r}>1$ is given by the simple relationship

$$
v_{\varphi}\left(x_{r}\right)=\frac{c}{x_{r}} .
$$

Further, if the magnetization parameter $\sigma$ exceeds the ratio $r_{\text {jet }} / R_{\mathrm{L}}$, the plasma Lorentz factor can again be estimated from the asymptotic solution (101), so that $\gamma \approx r_{\text {jet }} / R_{\mathrm{L}} \sim 10^{3}-10^{5}$. If $\sigma<r_{\text {jet }} / R_{\mathrm{L}}$, the effects of the finite mass of particles will limit their energy growth at large axial distances. To determine the magnetization parameter $\sigma$, as was shown, it is necessary to know the particle production multiplicity $\lambda$.

As noted above, it is impossible at present to determine the basic parameters of the outflowing plasma from observations. Nevertheless, some estimates can still be made. For example, one of the methods to determine the value of $\lambda$ is based on the assumption that the synchrotron radiation self-absorption occurs at the base of the jet [202]. This assumption allows one to estimate the particle number density [203]. On one-parsec scales, the characteristic particle number densities thus were found to be $10^{2}-10^{4} \mathrm{~cm}^{-3}$, which gives

$$
\lambda_{\mathrm{AGN}_{1}} \sim 10^{10}-10^{12} .
$$

If this indeed is the case, intensive secondary particle creation near the black hole horizon should have occurred. Here, according to relation (56) the value of $\sigma$ cannot exceed one hundred:

$$
\sigma_{\mathrm{AGN}_{1}} \sim 10^{2}-10^{3},
$$

which is much smaller than the ratio $r_{\text {jet }} / R_{\mathrm{L}} \sim 10^{5}$ corresponding to the maximum possible Lorentz factor derived from the asymptotic solution (101). Therefore, an almost complete transformation of the electromagnetic energy into particles' energy must occur in the process of collimation. Notice that in this case, although $\gamma_{\max }=\sigma \sim 10^{2}-10^{3}$ exceeds the particle energies that are required to explain the apparent superluminal motion effect, it is still insignificant. Here, almost all the energy flux in the jet will be related to the flux of accelerated particles. For $\sigma \sim 10^{2}-10^{3}$, the radius $r_{\mathrm{F}} \sim \sigma^{1 / 3} R_{\mathrm{L}}$ (66) of the fast magnetosonic surface must be smaller than one hundred radii from the central engine, which is $10^{14}-10^{16} \mathrm{~cm}$. Thus, the flow in the jet must indeed be supersonic.

Now making use of expressions (89), (95), and (96), we arrive at the conclusion that in this case all critical magnetic fields for the reasonable value of $\gamma_{\mathrm{in}} \sim 10$ are larger than $B_{\text {ext }} \sim 10^{-6} \mathrm{G}$ corresponding to the ambient pressure (for convenience, all parameters discussed here are listed below in Table 2). But this means that a denser core must exist in the center of the jet, and the subsonic flow near the axis will not be formed. At last, the ejection rate of electron-positron pairs, $\dot{N}=\pi R_{0}^{2} \cdot \lambda n_{\mathrm{GJ}} c$, can be estimated, which, as can be easily checked, is determined using the simple relation

$$
\dot{N} \sim \lambda\left(\frac{W_{\text {tot }} c}{e^{2}}\right)^{1 / 2} .
$$

As a result, we have $\dot{N}_{\mathrm{AGN}_{1}} \sim 10^{49}$ particles s ${ }^{-1}$ (hereinafter we set $\left.M=10^{9} M_{\odot}\right)$. In other words, about $10^{63}$ electron-positron pairs will be injected into host galaxy over the time of the active life of a galactic nucleus, $\tau \sim 10^{7}$ years. This number, incidentally, is quite sufficient to explain the intensity of the annihilation line emitted from the Galactic center, which, as is well known, requires about $10^{43}$ annihilations per second [204].

If the multiplicity factor of the secondary particle creation is small, $\lambda_{\mathrm{AGN}_{2}} \sim 10-100$, the inner structure of the jet must be significantly different, since now all critical fields are below the value of $10^{-6} \mathrm{G}$ corresponding to the ambient pressure. Here, one obtains

$$
\sigma_{\mathrm{AGN}_{2}} \sim 10^{10}-10^{12}
$$

so that the plasma Lorentz factor, according to the asymptotic solution (101), can be as high as approximately $10^{4}-10^{5}$, and a subsonic flow region must be formed in the center of the jet. In this case, the energy flux is Poynting-dominated. Correspondingly, the dense core will not be formed, so that both the poloidal magnetic field and the plasma density are weakly dependent on the axial distance. The electron-positron pair injection rate will be much smaller: $\dot{N}_{\mathrm{AGN}_{2}} \sim 10^{39}$ particles $\mathrm{s}^{-1}$. However, in this case, too, the fast magnetosonic surface radius $r_{\mathrm{F}} \sim 10^{17}-10^{18} \mathrm{~cm}$ will be smaller than the jet transverse size. Notice that here there is no direct contradiction with observations, since, as has been noted, the drift motion of particles will be directed almost along the poloidal magnetic field. This particle motion does not produce synchrotron radiation. Hence, 
one should be cautious in using the standard synchrotron radiation formulas to estimate the value of the magnetic field and the lifetime of relativistic particles.

We should keep in mind that, when estimating radiation from relativistic jets, one usually assumes that an approximate equipartition between the energy densities of particles and the magnetic field takes place $\left(B^{(\mathrm{com}) 2} / 8 \pi \sim \gamma^{(\mathrm{com})} n^{(\mathrm{com})} m c^{2}\right)$ in the rest frame of plasma. The parameters we introduced allow us to obtain simple relationships for all quantities in this reference frame. In particular, it is easy to show that the characteristic Lorentz factor of particles in the plasma rest frame is simply the following:

$$
\gamma^{(\mathrm{com})} \approx \frac{\sigma}{\gamma}
$$

where $\gamma$ is the bulk Lorentz factor of the hydrodynamic flow. On the other hand, one finds $B^{(\mathrm{com})} \approx\left(x_{r} / \gamma\right) B_{\mathrm{p}}$. Consequently, we have $B^{(\mathrm{com})} \approx B_{\mathrm{p}}$ in the effective acceleration region $\left(\gamma=x_{r}\right)$, while for the asymptotic solution (102) we obtain $B^{(\mathrm{com})} \gg B_{\mathrm{p}}$.

\subsection{Microquasars}

If the operation of the central engine in microquasars indeed can be described by the MHD model considered here, it is again possible to assume that the observed subrelativistic jet velocities must correspond to $\sigma$ on the order of 3-10. Then, according to relation (56), we should conclude that the particle production multiplicity in the microquasar magnetosphere must be fairly large:

$$
\lambda_{\mu \mathrm{QSO}} \sim 10^{10} .
$$

Such a high value is also supported by the compactness parameter $l_{a, \mu \mathrm{QSO}} \sim 10^{4}$. Then, the electronpositron pair ejection rate should be $\dot{N}_{\mu \mathrm{QSO}} \sim 10^{43}$ particles $\mathrm{s}^{-1}$. Finally, the large values of the critical fields $\left(B_{\text {min }} \sim 10^{4} \mathrm{G}, B_{\text {eq }} \sim 10^{3} \mathrm{G}, B_{\text {cr }} \sim 10^{6} \mathrm{G}\right)$ indicate that a dense core must exist in the jet center, and the subsonic flow near the rotational axis does not form.

On the other hand, if $\sigma$ is indeed not too high, the fast magnetosonic surface $\left(r_{\mathrm{F}} \sim \sigma^{1 / 3} R_{\mathrm{L}}\right)$ must lie close to the light cylinder, i.e., at distances of order $10^{7}-10^{8} \mathrm{~cm}$. This scale is much smaller than the distance from the central engine to the companion star in binaries involving a microquasar. Hence, we can conclude that the interaction of the stellar wind and matter ejected from the microquasar occurs in the supersonic regime.

\subsection{Sources of cosmological gamma-ray bursts}

Now let us see which parameters can characterize jets outflowing cosmological gamma-ray bursts. It should be recalled that, according to one of the most popular models, a rapidly rotating central engine (magnetar, black hole) is immersed in the progenitor massive star
[40]. That is why, the ambient pressure for the jet in its formation region is provided not by the surrounding medium with very small pressure, but by the matter of the massive star itself (the equivalent magnetic field $B_{\text {ext }} \sim 10^{6}-10^{8} \mathrm{G}$ ). Notice also that in cosmological gamma-ray bursts there can be one more mechanism of the electron-positron pair creation, related to neutrino annihilation. Such neutrinos can be copiously created during supercritical accretion onto the collapsing stellar core [205].

The starting point that can shed light on the physical conditions inside the central engine can be the characteristic particle Lorentz factor $\gamma \sim 300$, which in this case can be naturally related to the magnetization parameter $\sigma:$

$$
\sigma_{\mathrm{GRB}} \sim 10^{2}-10^{3} .
$$

If the condition $\gamma \ll \sigma$ is satisfied, the total energy release from the central engine would be unrealistically high. Now, using Eqn (56) to estimate the plasma generation multiplicity, we obtain

$$
\lambda_{\mathrm{GRB}} \sim 10^{13}-10^{14} .
$$

Such a huge value unambiguously evidences that the particle creation efficiency must be high enough. Indeed, formula (57) gives very large compactness parameter $l_{a} \sim 10^{15}$. Correspondingly, for the electron-positron pair ejection rate we find $\dot{N}_{\mathrm{GRB}} \sim 10^{53}$ particles s $^{-1}$.

Next, the very small sizes of the central engine together with the moderate value of the magnetization parameter $\sigma$ shows that the fast magnetosonic surface radius $r_{\mathrm{F}}(66)$ should not exceed $10^{7}-10^{8} \mathrm{~cm}$, which is significantly smaller than the size of the progenitor star. Consequently, the matter outflow in the jet becomes supersonic before it exits the star. Finally, expressions (89) and (95) for the characteristic magnetic fields $B_{\min }$ and $B_{\text {eq }}$ indicate that they are in the range of $10^{6}-10^{8} \mathrm{G}$, i.e., their pressure is comparable with that inside the progenitor star. Therefore, the jet transverse size will indeed be sufficient to accelerate particles up to energies $\gamma \sim \sigma$.

Notice at last that the condition $\gamma \vartheta \sim 1$ is certainly not satisfied for gamma-ray bursts, since in that case the jet spread angles would only be $0.1^{\circ}$, while observations indicate that $\vartheta \sim 1-10^{\circ}[206]$. Such a flow can also be realized. For example, it was shown in paper [207] (see also Ref. [208]) that in the model of an infinitely extended progenitor star, where the ambient pressure decreases graduately according to a power law, the acceleration turns out to be not very effective in comparison with a more realistic model in which the ambient pressure beyond the star is assumed to be low. As it has turned out, in both cases the flow corresponds to a weakly collimated flux with $1<k<2$, where the particle energy follows the asymptotic behaviour $\gamma \approx\left(R_{\mathrm{c}} / \varpi\right)^{1 / 2}(102)$. In the former case, however, the radius of curvature $R_{\mathrm{c}}$ of the magnetic surfaces, which is determined by the pressure decrease law inside the progenitor star, turns out to be sufficiently small, which precludes plasma from being effectively accelerated. Beyond the star, magnetic field lines straighten up (and hence the radius of curvature 
increases), which leads to a more effective acceleration. As we see, the simple analytical asymptotic solutions obtained above allow easy interpretation of the numerical experiment.

\subsection{Radio pulsars}

Radio pulsars, undoubtedly, only indirectly relate to the topic considered here, since their magnetospheres are certainly not axisymmetric and stationary. It is not then surprising that jets, as we have noted, are observed only from two energetic pulsars. Nevertheless, many points, which were possible to clarify in the theory of the neutron star magnetosphere, undoubtedly allow us to shed light on the nature of other compact objects, too.

First and foremost, it should be noted that basic parameters characterizing the pulsar wind are well known inside the light cylinder. This is due to our good knowledge of the process of plasma creation near the magnetic polar caps of a neutron star. Numerous calculations have shown that the general properties of the secondary electron-positron plasma flowing out from the magnetosphere turned out to be only a little sensitive to the details of the acceleration region structure. For most models $[178,180,209,210]$, both the density and the energy spectrum of the plasma flowing out are universal enough. Therefore, with certainty we can say that the plasma flowing along open field lines in the pulsar magnetosphere includes both the primary particle beam with energy $\mathcal{E} \approx 10^{7} \mathrm{MeV}$ and density close to the Goldreich density and the secondary electron-positron component. Its energy spectrum with a good accuracy exhibits a power law dependence

$$
\mathcal{E} \propto \mathcal{E}^{-2},
$$

ranging from $\mathcal{E}_{\min } \sim 10-100 \mathrm{MeV}$ to $\mathcal{E}_{\max } \sim 10^{4} \mathrm{MeV}$. The total density of the secondary plasma for ordinary pulsars exceeds the Goldreich density by $10^{3}-10^{4}$ times. And only for the most energetic pulsars can the multiplicity factor reach $10^{5}$.

Thus, the parameter $\lambda$ for radio pulsars is determined quite reliably:

$$
\lambda_{\mathrm{PSR}} \sim 10^{3}-10^{4} .
$$

Now, making use again of relation (56) we obtain

$$
\sigma_{\mathrm{PSR}} \sim 10^{3}-10^{4} .
$$

And only for the most energetic pulsars do we find $\sigma_{\text {PSR }} \sim 10^{5}-10^{6}$. Thus, the condition $\gamma_{\text {in }} \gg \sigma^{1 / 3}$ is satisfied in the vast majority of pulsars, which corresponds to a slow rotation of the central object [99]. Indeed, the equality $\gamma_{\text {in }}=\sigma^{1 / 3}$ can be written out in the form $P=P_{\text {cr }}$, where

$$
\begin{array}{r}
P_{\mathrm{cr}}=\pi \frac{R}{c}\left[\frac{2}{\lambda \gamma_{\mathrm{in}}^{3}}\left(\frac{\omega_{B} R}{c}\right)\right]^{1 / 2} \\
\sim 10^{-3}\left(\frac{\lambda}{10^{4}}\right)^{-1 / 2}\left(\frac{\gamma}{10^{2}}\right)^{-3 / 2}\left(\frac{B_{0}}{10^{12} \mathrm{G}}\right)^{1 / 2} \mathrm{~s} .
\end{array}
$$

For fast rotation $\left(P \ll P_{\mathrm{cr}}\right)$, the particle energy significantly increases when particles approach the fast magnetosonic surface, whereas for slowly rotating objects $\left(P \gg P_{\text {cr }}\right.$ the particle energy remains practically unchanged. Their further fate, as we have shown above, depends on whether the flow intersects the light surface or not.

\subsection{Young stars}

In conclusion, we discuss the main parameters which characterize nonrelativistic jets from young stars. It should be recalled that in this case the nonrelativistic magnetization parameter an $\sigma_{\mathrm{n}} \approx\left(\Omega_{\mathrm{F}} / \Omega_{\mathrm{cr}}\right)^{2}(53)$ plays the key role. Under the condition $\sigma_{\mathrm{n}} \gg 1\left(\Omega_{\mathrm{F}} \gg \Omega_{\mathrm{cr}}\right)$, the electromagnetic energy flux near the central engine will be much greater than the particle energy flux; beyond singular surfaces, as stressed above, particles must carry a significant fraction of the total energy.

As the critical period $P_{\mathrm{cr}}=2 \pi / \Omega_{\mathrm{cr}}$, namely

$$
\begin{aligned}
& P_{\text {cr }} \approx 10\left(\frac{R}{10^{11} \mathrm{~cm}}\right)^{2}\left(\frac{B_{0}}{10^{3} \mathrm{G}}\right)\left(\frac{r_{\mathrm{d}} / R}{30}\right)^{-1} \\
& \left(\frac{v_{\mathrm{in}}}{100 \mathrm{~km} \mathrm{~s}^{-1}}\right)^{-3 / 2}\left(\frac{\dot{M}}{10^{-9} M_{\odot} \mathrm{yr}^{-1}}\right)^{-1 / 2} \mathrm{~d}
\end{aligned}
$$

( $r_{\mathrm{d}}$ is the inner radius of the accretion disc) is close to the spin periods of young stars, in the region of magnetic field lines coming out of the surface of the star we have $\sigma_{\mathrm{n}} \sim 1$. On the other hand, the period $P_{\mathrm{cr}}$ is one two orders of magnitude larger than the rotation periods in the inner regions of accretion discs, $P_{\mathrm{b}}=2 \pi\left(G M / r^{3}\right)^{1 / 2}$, so that for the corresponding magnetic field lines one finds $\sigma_{\mathrm{n}} \sim 10-1000$. That is why, the inner parts of the accretion disc, not the central star, must play the role of the central engine rotor. As noted above, there are observational evidences of this being the case [76].

Next, from relation (66) we obtain

$$
r_{\mathrm{A}} \sim r_{\mathrm{F}} \sim \frac{v_{\text {in }}}{\Omega} \sigma_{\mathrm{n}}^{1 / 3},
$$

so that $r_{\mathrm{F}} \sim 10-30 R$. Thus, the distance to singular surfaces exceeds the size of the star by 10-30 times, but it is the same 10-30 times smaller than the transverse size of jets. Consequently, the flow inside the jets must be supersonic and, hence, the longitudinal current for these objects again must be derived from the critical conditions on the singular surfaces. Correspondingly, the radius of the jet core must be on the order of $r_{\text {core }}=v_{\text {in }} / \Omega \sim 0.1$ a.u., the jet magnetic field should be as high as $B_{\text {core }} \sim 0.1\left(\Omega r_{\text {core }} / v_{\text {in }}\right)^{2} B_{\text {in }} \sim 10^{-2} \mathrm{G}$, and the particle number density must range from $10^{8}$ to $10^{9} \mathrm{~cm}^{-3}$.

Finally, we note that the existence of the integrals of motion allows us to obtain direct information about the plasma outflow region. For example, if the radial and longitudinal velocities of the flow in the jet are known at the axial distance $r_{\perp}$ (and such observations, as noted 
above, have already been performed for several young stars $[72,73])$, it is possible to estimate the distance $r_{\text {st }}$ from the central star at which the corresponding force line is 'anchored' in the accretion disc [211, 212]:

$$
\begin{aligned}
r_{\mathrm{st}} \approx & 0.7\left(\frac{r_{\perp}}{10 \mathrm{a} . \mathrm{u} .}\right)^{2 / 3}\left(\frac{v_{\varphi}}{10 \mathrm{~km} \mathrm{~s}^{-1}}\right)^{2 / 3} \\
& \left(\frac{v_{\mathrm{p}}}{100 \mathrm{~km} \mathrm{~s}^{-1}}\right)^{-4 / 3}\left(\frac{M}{M_{\odot}}\right)^{1 / 3} \text { a.u. }
\end{aligned}
$$

It is obvious that this scale is much larger than the size of the star, so at present it has been possible to resolve only outermost regions of the outflow.

\section{Conclusions}

Thus, significant progress has indeed been recently achieved in the understanding of the nature of jets observed in different classes of astrophysical sources. This became possible because the analytical approach allowed sufficiently simple relationships between physical parameters characterizing the outflows to be found, and the numerical modeling (in which, we should keep in mind, the setup of the problem itself has been significantly different from the analysis of stationary equations) has confirmed these relationships.

The most important result of the analytical theory includes the understanding of the role of key dimensionless parameters. For clearness, they are listed in Table 2 (for active galactic nuclei we set $M=10^{9} M_{\odot}$ ). It turned out that the knowledge of these parameters allows us to estimate many characteristics of jets, including the fraction of energy carried by particles, the plasma Lorentz factor, the electron positron pair injection rate $\dot{N}$, and the compactness parameter $l_{a}$, as well as to determine the main parameters of the internal structure of jets. The determination of these parameters from observations would be a significant breakthrough in our understanding of physical processes which are underway in active astrophysical sources.

Next, we have shown that many properties of relativistic and nonrelativistic jets are significantly different from each other. For convenience, we also collect them together in Table 3. As can be seen, one should be very cautious when applying the results, which were obtained for nonrelativistic jets, to ultrarelativistic flows. Moreover, the asymptotic relations formulated above clarify many results obtained by means of numerical simulations.

The limited space of the present review did not allow us to discuss in detail many important issues. In particular, we only briefly discussed the stability of jets. Finally, here we have no space at all to discuss the nonstationary performance of the central engine (most papers have recently started focusing exactly on this topic) or the proper radiation of jets. Nevertheless, we would like to hope that questions addressed in this review will be useful for future studies of relativistic and nonrelativistic outflows observed in many astrophysical objects.
Table 2: Parameters of jets outflowing from relativistic compact objects. All values are given to an order of magnitude.

\begin{tabular}{|l|c|c|c|c|c|}
\hline & $\mathrm{AGN}_{1}$ & $\mathrm{AGN}_{2}$ & $\mu \mathrm{QSO}$ & $\mathrm{GRB}$ & $\mathrm{PSR}$ \\
\hline$\sigma$ & 100 & $10^{12}$ & 10 & $10^{3}$ & $10^{4}$ \\
$\lambda$ & $10^{12}$ & 100 & $10^{10}$ & $10^{14}$ & $10^{3}$ \\
$\gamma_{\text {in }}$ & 10 & 10 & 10 & 10 & 100 \\
\hline$l_{a}$ & $1-100$ & $1-100$ & $10^{4}$ & $10^{15}$ & $10^{-5}$ \\
$W_{\text {part }} / W_{\text {tot }}$ & 1 & $10^{-9}$ & $10^{-5}$ & 1 & $10^{-2}$ \\
$\gamma$ & $10^{-100}$ & $10^{4}-10^{5}$ & $10^{3}$ & 300 & $10^{3}$ \\
$\dot{N}_{, \mathrm{s}^{-1}}$ & $10^{49}$ & $10^{39}$ & $10^{33}$ & $10^{53}$ & $10^{32}$ \\
\hline$B_{\min }, \mathrm{G}$ & $10^{-2}$ & $10^{-12}$ & $10^{4}$ & $10^{8}$ & $10^{-6}$ \\
$B_{\text {eq }}, \mathrm{G}$ & $10^{-4}$ & $10^{-24}$ & $10^{3}$ & $10^{6}$ & $10^{-8}$ \\
$B_{\mathrm{cr}}, \mathrm{G}$ & $10^{-1}$ & $10^{-11}$ & $10^{6}$ & $10^{10}$ & $10^{-2}$ \\
\hline
\end{tabular}

\section{Acknowledgments}

I would like to acknowledge M Barkov, S Komissarov, M Romanova, and A D Tchekhovskoy for carefully reading the manuscript and many notes, and also A V Gurevich, Ya N Istomin, Yu Yu Kovalev, R Lovelace, Yu Lyubarsky, and A Spitkovsky for useful discussions. The work was supported by RFBR grant 09-02-00749 and the Federal Agency of Science and Innovations (contract No. 02.740.11.0250).

\section{Appendix}

In the Appendix, we give for reference the complete set of equations of the Grad-Shafranov method written out in themost general case, i.e., for axisymmetric stationary flows in the vicinity of a rotating black hole. First of all, we keep in mind the basic relations for the Kerr metric of a rotating black hole. In the Boyer-Lindquist coordinates $t, r, \theta$, and $\varphi$, it assumes the form

$$
\mathrm{d} s^{2}=-\alpha^{2} \mathrm{~d} t^{2}+g_{i k}\left(\mathrm{~d} x^{i}+\beta^{i} \mathrm{~d} t\right)\left(\mathrm{d} x^{k}+\beta^{k} \mathrm{~d} t\right),
$$

where the quantity

$$
\alpha=\frac{\rho}{\Sigma} \sqrt{\Delta}
$$

is the gravitational redshift, and the vector $\beta$ is toroidal:

$$
\beta^{r}=\beta^{\theta}=0, \quad \beta^{\varphi}=-\omega .
$$

Here

$$
\omega=\frac{2 a M r}{\Sigma^{2}}
$$

is the so-called Lense-Thirring angular velocity. Finally, $M$ and $a$ are the mass and specific angular momentum of the black hole $(a=J / M)$, respectively. In addition, we introduced the standard notations

$$
\begin{gathered}
\Delta=r^{2}+a^{2}-2 M r, \quad \rho^{2}=r^{2}+a^{2} \cos ^{2} \theta, \\
\Sigma^{2}=\left(r^{2}+a^{2}\right)^{2}-a^{2} \Delta \sin ^{2} \theta, \quad \varpi=\frac{\Sigma}{\rho} \sin \theta .
\end{gathered}
$$


Table 3: Main differences between relativistic and nonrelativistic jets.

\begin{tabular}{|l|l|}
\hline Relativistic flow & Nonrelativistic flow \\
\hline Longitudinal current is close to the Goldreich current & $\begin{array}{l}\text { Longitudinal current is much larger than the } \\
\text { Goldreich current }\end{array}$ \\
\hline $\begin{array}{l}\text { For strongly magnetized flow } \sigma \gg 1) \text {, fast } \\
\text { magnetosonic surface near equatorial plane is located } \\
\sigma^{1 / 3} \text { times farther than Alfvénic surface }\end{array}$ & $\begin{array}{l}\text { Fast magnetosonic surface is located near Alfvénic } \\
\text { surface }\end{array}$ \\
\hline $\begin{array}{l}\text { On fast magnetosonic surface, the particle energy flux } \\
\text { is much smaller than the electromagnetic energy flux }\end{array}$ & $\begin{array}{l}\text { On fast magnetosonic surface, the particle energy flux } \\
\text { is close to the electromagnetic energy flux }\end{array}$ \\
\hline $\begin{array}{l}\text { Beyond Alfvénic surface, the electric field is close } \\
\text { in magnitude to the magnetic field }\end{array}$ & $\begin{array}{l}\text { Beyond Alfvénic surface, the electric field is much } \\
\text { smaller than the magnetic field }\end{array}$ \\
\hline $\begin{array}{l}\text { Proper collimation is impossible for both strongly and } \\
\text { weakly magnetized outflows }\end{array}$ & $\begin{array}{l}\text { Proper collimation becomes effective for strongly } \\
\text { magnetized outflows }\end{array}$ \\
\hline $\begin{array}{l}\text { The dense core in jets can be formed only under } \\
\text { sufficiently small ambient pressures. Magnetic flux } \\
\text { in the core is much smaller than the total flux }\end{array}$ & $\begin{array}{l}\text { The dense core is always formed in jets. Magnetic flux } \\
\text { in the core is a significant fraction of the total } \\
\text { magnetic flux }\end{array}$ \\
\hline Cylindrical flow with subsonic core is possible & Cylindrical flow with subsonic core is impossible \\
\hline
\end{tabular}

Here, in all relativistic expressions we use the units in which $c=G=1$. Finally, it is important that the threedimensional metric $g_{i k}$ in formula (A.1) be diagonal:

$$
g_{r r}=\frac{\rho^{2}}{\Delta}, \quad g_{\theta \theta}=\rho^{2}, \quad g_{\varphi \varphi}=\varpi^{2} .
$$

As for the flat space limit, it can be easily obtained by passing to the limit $\alpha \rightarrow 1$ and $\omega \rightarrow 0$.

As is well known, for calculations it is convenient to introduce a special reference frame, the so-called ZAMO (Zero Angular Momentum Observers) [131], which has the following properties:

- ZAMO observers are located at constant radius $r=$ const, $\theta=$ const but rotate with the Lense Thirring angular velocity $\mathrm{d} \varphi / \mathrm{d} t=\omega$;

- for ZAMO, the four-dimensional metric $g_{\alpha \beta}$ is diagonal, with its three-dimensional part $g_{i k}$ coinciding with Eqn $(A .6)$.

Below, all vectors will be written out in this reference frame. In particular, the operator $\nabla_{i}$, means the covariant derivative in the three-dimensional metric (A.6).

As a result, the electric and magnetic fields can be conveniently written as

$$
\begin{gathered}
\mathbf{B}=\frac{\nabla \Psi \times \mathbf{e}_{\hat{\varphi}}}{2 \pi \varpi}-\frac{2 I}{\alpha \varpi} \mathbf{e}_{\hat{\varphi}}, \\
\mathbf{E}=-\frac{\Omega_{\mathrm{F}}-\omega}{2 \pi \alpha} \nabla \Psi,
\end{gathered}
$$

respectively, and the four-velocity of matter is written as

$$
\mathbf{u}=\frac{\eta}{\alpha n} \mathbf{B}+\gamma\left(\Omega_{\mathrm{F}}-\omega\right) \frac{\varpi}{\alpha} \mathbf{e}_{\hat{\varphi}},
$$

where $\gamma=1 / \sqrt{1-v^{2}}$ is the Lorentz factor of matter, and the subscripts with a cap over them correspond to physical components of vectors. The quantity $\Omega_{\mathrm{F}}$ remains the integral of motion. In turn, integrals of motions $E$ and $L$ will be written out as

$$
\begin{gathered}
E=E(\Psi)=\frac{\Omega_{\mathrm{F}} I}{2 \pi}+\mu \eta\left(\alpha \gamma+\omega \varpi u_{\hat{\varphi}}\right), \\
L=L(\Psi)=\frac{I}{2 \pi}+\mu \eta \varpi u_{\hat{\varphi}} .
\end{gathered}
$$

Next, the relativistic Bernoulli equation $\gamma^{2}-u_{\hat{\varphi}}^{2}=u_{\mathrm{p}}^{2}+1$ takes the form

$$
\frac{K}{\varpi^{2} A^{2}}=\frac{1}{64 \pi^{4}} \frac{\mathcal{M}^{4}(\nabla \Psi)^{2}}{\varpi^{2}}+\alpha^{2} \eta^{2} \mu^{2},
$$

where the Alfvén factor is

$$
A=\alpha^{2}-\left(\Omega_{\mathrm{F}}-\omega\right)^{2} \varpi^{2}-\mathcal{M}^{2},
$$

and

$$
\begin{gathered}
K=\alpha^{2} \varpi^{2}\left(E-\Omega_{\mathrm{F}} L\right)^{2}\left[\alpha^{2}-\left(\Omega_{\mathrm{F}}-\omega\right)^{2} \varpi^{2}-2 \mathcal{M}^{2}\right] \\
+\mathcal{M}^{4}\left[\varpi^{2}(E-\omega L)^{2}-\alpha^{2} L^{2}\right] .
\end{gathered}
$$

This equation defines the Alfvénic Mach number M, where

$$
\mathcal{M}^{2}=\frac{4 \pi \eta^{2} \mu}{n} .
$$

Now, making use of relations $(A .12)-(A .14)$, which can be recast in the form $(\nabla \Psi)^{2}=F\left(\mathcal{M}^{2}, E, L, \eta, \Omega_{\mathrm{F}}, \mu\right)$, where

$$
F=\frac{64 \pi^{4}}{\mathcal{M}^{4}} \frac{K}{A^{2}}-\frac{64 \pi^{4}}{\mathcal{M}^{4}} \alpha^{2} \varpi^{2} \eta^{2} \mu^{2},
$$

we obtain

$$
\nabla_{k} \mathcal{M}^{2}=\frac{N_{k}}{D}
$$


where

$$
N_{k}=-\frac{A}{(\nabla \Psi)^{2}} \nabla^{i} \Psi \cdot \nabla_{i} \nabla_{k} \Psi+\frac{A}{2} \frac{\nabla_{k}^{\prime} F}{(\nabla \Psi)^{2}} .
$$

Here, the operator $\nabla_{k}^{\prime}$ acts on all quantities but $\mathcal{M}^{2}$. The quantity $\nabla_{k}^{\prime} \mu$ must be determined from the relation [97]

$$
\nabla_{k}^{\prime} \mu=\frac{2 c_{\mathrm{s}}^{2}}{1-c_{\mathrm{s}}^{2}} \mu \frac{\nabla_{k} \eta}{\eta}+\frac{1}{1-c_{\mathrm{s}}^{2}}\left[\frac{1}{n}\left(\frac{\partial P}{\partial s}\right)_{n}+T\right] \nabla_{k} s,
$$

where $c_{\mathrm{s}}^{2}=1 / \mu(\partial P / \partial n)_{s}$ is the speed of sound, and $s$ is the entropy. In turn, the denominator $D$ can be rewritten in the form

$$
D=\frac{A}{\mathcal{M}^{2}}+\frac{\alpha^{2}}{\mathcal{M}^{2}} \frac{B_{\hat{\varphi}}^{2}}{B_{\mathrm{p}}^{2}}-\frac{1}{u_{\mathrm{p}}^{2}} \frac{A}{\mathcal{M}^{2}} \frac{c_{\mathrm{s}}^{2}}{1-c_{\mathrm{s}}^{2}} .
$$

As for the Grad-Shafranov equation, in the compact form it can be written out as $[96,97]$

$$
\begin{gathered}
\frac{1}{\alpha} \nabla_{k}\left\{\frac{1}{\alpha \varpi^{2}}\left[\alpha^{2}-\left(\Omega_{\mathrm{F}}-\omega\right)^{2} \varpi^{2}-\mathcal{M}^{2}\right] \nabla^{k} \Psi\right\} \\
+\frac{\Omega_{\mathrm{F}}-\omega}{\alpha^{2}}(\nabla \Psi)^{2} \frac{\mathrm{d} \Omega_{\mathrm{F}}}{\mathrm{d} \Psi} \quad(A .21) \\
+\frac{64 \pi^{4}}{\alpha^{2} \varpi^{2}} \frac{1}{2 \mathcal{M}^{2}} \frac{\partial}{\partial \Psi}\left(\frac{G}{A}\right)-16 \pi^{3} \mu n \frac{1}{\eta} \frac{\mathrm{d} \eta}{\mathrm{d} \Psi}-16 \pi^{3} n T \frac{\mathrm{d} s}{\mathrm{~d} \Psi}=0,
\end{gathered}
$$

where

$$
G=\alpha^{2} \varpi^{2}\left(E-\Omega_{\mathrm{F}} L\right)^{2}+\alpha^{2} \mathcal{M}^{2} L^{2}-\mathcal{M}^{2} \varpi^{2}(E-\omega L)^{2} .
$$

Now, expanding terms $\nabla_{k} \mathcal{M}^{2}$ in Eqn $(A .21)$ according to definitions $(A .17)-(A .19)$, we finally arrive at

$$
\begin{gathered}
A\left[\frac{1}{\alpha} \nabla_{k}\left(\frac{1}{\alpha \varpi^{2}} \nabla^{k} \Psi\right)+\frac{1}{\alpha^{2} \varpi^{2}(\nabla \Psi)^{2}} \frac{\nabla^{i} \Psi \cdot \nabla^{k} \Psi \cdot \nabla_{i} \nabla_{k} \Psi}{D}\right] \\
+\frac{1}{\alpha^{2} \varpi^{2}} \nabla_{k}^{\prime} A \cdot \nabla^{k} \Psi-\frac{A}{\alpha^{2} \varpi^{2}(\nabla \Psi)^{2}} \frac{1}{2 D} \nabla_{k}^{\prime} F \cdot \nabla^{k} \Psi \\
+\frac{\Omega_{\mathrm{F}}-\omega}{\alpha^{2}}(\nabla \Psi)^{2} \frac{\mathrm{d} \Omega_{\mathrm{F}}}{\mathrm{d} \Psi}+\frac{64 \pi^{4}}{\alpha^{2} \varpi^{2}} \frac{1}{2 \mathcal{M}^{2}} \frac{\partial}{\partial \Psi}\left(\frac{G}{A}\right) \\
-16 \pi^{3} \mu n \frac{1}{\eta} \frac{\mathrm{d} \eta}{\mathrm{d} \Psi}-16 \pi^{3} n T \frac{\mathrm{d} s}{\mathrm{~d} \Psi}=0,
\end{gathered}
$$

where again the gradient $\nabla_{k}^{\prime}$ acts on all quantities but $\mathcal{M}^{2}$, and the derivative $\partial / \partial \Psi$ acts only onh the integrals of motion. Formula (A27) determines in the most general form the equilibrium equation for the magnetic surfaces. Finally algebraic relations have the form

$$
\begin{gathered}
\frac{I}{2 \pi}=\frac{\alpha^{2} L-\left(\Omega_{\mathrm{F}}-\omega\right) \varpi^{2}(E-\omega L)}{\alpha^{2}-\left(\Omega_{\mathrm{F}}-\omega\right)^{2} \varpi^{2}-\mathcal{M}^{2}}, \\
\gamma=\frac{1}{\alpha \mu \eta} \frac{\alpha^{2}\left(E-\Omega_{\mathrm{F}} L\right)-\mathcal{M}^{2}(E-\omega L)}{\alpha^{2}-\left(\Omega_{\mathrm{F}}-\omega\right)^{2} \varpi^{2}-\mathcal{M}^{2}},
\end{gathered}
$$

$$
u_{\hat{\varphi}}=\frac{1}{\varpi \mu \eta} \frac{\left(E-\Omega_{\mathrm{F}} L\right)\left(\Omega_{\mathrm{F}}-\omega\right) \varpi^{2}-L \mathcal{M}^{2}}{\alpha^{2}-\left(\Omega_{\mathrm{F}}-\omega\right)^{2} \varpi^{2}-\mathcal{M}^{2}} .
$$

Equations (A.12) and (A.24)-(A.26) represent algebraic bounds which allow the determination, albeit in an indirect form, of all characteristics of the flow from the given poloidal field $\mathbf{B}_{\mathrm{p}}$ (i.e., from the known potential $\Psi$ ) and five integrals of motion. It should be emphasized that for a nonzero temperature they are extremely lengthy, mainly due to the need to resolve equation (A.19). In the case of cold plasma $(s=0$, i.e., $\mu=$ const), Bernoulli equation (A.12) becomes a fourthorder algebraic equation with respect to $\mathcal{M}^{2}$. As shown above, this fact often allows analytical asymptotics to be found.

In the cylindrical case, the second-order GradShafranov equation can be conveniently reduced to the system of two ordinary differential equations of the first order for the magnetic flux $\Psi(\varpi)$ and the Mach number $\mathcal{M}^{2}$. The equation for the Mach number has therewith the form [146]

$$
\begin{gathered}
{\left[\frac{\left(e^{\prime}\right)^{2}}{\mu^{2} \eta^{2}}-1+\frac{\Omega_{\mathrm{F}}^{2} r^{2}}{c^{2}}-A \frac{c_{\mathrm{s}}^{2}}{c^{2}}\right] \frac{\mathrm{d} \mathcal{M}^{2}}{\mathrm{~d} r}=\frac{\mathcal{M}^{6} L^{2}}{A r^{3} \mu^{2} \eta^{2} c^{2}}} \\
+\frac{\Omega_{\mathrm{F}}^{2} r \mathcal{M}^{2}}{c^{2}}\left[2-\frac{\left(e^{\prime}\right)^{2}}{A \mu^{2} \eta^{2} c^{4}}\right]+\mathcal{M}^{2} \frac{e^{\prime}}{\mu^{2} \eta^{2} c^{4}} \frac{\mathrm{d} \Psi}{\mathrm{d} r} \frac{\mathrm{d} e^{\prime}}{\mathrm{d} \Psi} \\
+\mathcal{M}^{2} \frac{r^{2}}{c^{2}} \Omega_{\mathrm{F}} \frac{\mathrm{d} \Psi}{\mathrm{d} r} \frac{\mathrm{d} \Omega_{\mathrm{F}}}{\mathrm{d} \Psi}-\mathcal{M}^{2}\left(1-\frac{\Omega_{\mathrm{F}}^{2} r^{2}}{c^{2}}+2 A \frac{c_{\mathrm{s}}^{2}}{c^{2}}\right) \frac{\mathrm{d} \Psi}{\mathrm{d} r} \frac{1}{\eta} \frac{\mathrm{d} \eta}{\mathrm{d} \Psi} \\
-\left[\frac{A}{n}\left(\frac{\partial P}{\partial s}\right)_{n}+\left(1-\frac{\Omega_{\mathrm{F}}^{2} r^{2}}{c^{2}}\right) T\right] \frac{\mathcal{M}^{2}}{\mu} \frac{\mathrm{d} \Psi}{\mathrm{d} r} \frac{\mathrm{d} s}{\mathrm{~d} \Psi}, \quad(A .27)
\end{gathered}
$$

where $e^{\prime}=E-\Omega_{\mathrm{F}} L$. The equation for the magnetic flux $\Psi$ will coincide with Bernoulli equation (A.14). Finally, the force-free pulsar equation takes on the form

$$
\begin{array}{r}
-\left(1-\frac{\Omega_{\mathrm{F}}^{2} \varpi^{2}}{c^{2}}\right) \nabla^{2} \Psi+\frac{2}{\varpi} \frac{\partial \Psi}{\partial \varpi} \\
-\frac{16 \pi^{2}}{c^{2}} I \frac{\mathrm{d} I}{\mathrm{~d} \Psi}+\frac{\varpi^{2}}{c^{2}}(\nabla \Psi)^{2} \Omega_{\mathrm{F}} \frac{\mathrm{d} \Omega_{\mathrm{F}}}{\mathrm{d} \Psi}=0,
\end{array}
$$

where $\nabla^{2}$ is the Laplace operator. Its generalization to the force-free black hole magnetosphere is written as [131]

$$
\begin{array}{r}
\quad \frac{1}{\alpha} \nabla_{k}\left\{\frac{\alpha}{\varpi^{2}}\left[1-\frac{\left(\Omega_{\mathrm{F}}-\omega\right)^{2} \varpi^{2}}{\alpha^{2}}\right] \nabla^{k} \Psi\right\} \\
+\frac{\Omega_{\mathrm{F}}-\omega}{\alpha^{2}}(\nabla \Psi)^{2} \frac{\mathrm{d} \Omega_{\mathrm{F}}}{\mathrm{d} \Psi}+\frac{16 \pi^{2}}{\alpha^{2} \varpi^{2}} I \frac{\mathrm{d} I}{\mathrm{~d} \Psi}=0 .
\end{array}
$$

These equations are elliptical in all the space, and so they require boundary conditions on the integration region boundary or on the black hole horizon. 


\section{References}

1. Kardashev N S Astron. Zh. 41807 (1964) [ Sov. Astron. 8643 (1965)]

2. Pacini V Nature 216567 (1967)

3. Goldreich P, Julian W H Astrophys. J. 157869 (1969)

4. Michel F C Astrophys. J. 158727 (1969)

5. Blandford R D Mon. Not. R. Astron. Soc. 176465 (1976)

6. Lovelace R W ENature 262649 (1976)

7. Bisnovatyi-Kogan G S, Popov Yu P, Samokhin A A Astrophys. Space Sci. 41321 (1976)

8. Moiseenko S G, Bisnovatyi-Kogan G S, Ardeljan N V Astrophys. J. 370501 (2006)

9. Beskin V S Osesimmetrichnye Statsionamye Techeniya $v$ Astrofizike (MHD Flows in Compact Astrophysical Objects) (Moscow: Fizmatlit, 2005) [Translated into English (Berlin: Springer, 2009)]

10. Beskin V S Usp. Fiz. Nauk 167689 (1997) [Phys. Usp. 40659 (1997)]

11. Shapiro S, Teukolsky S Black Holes, White Dwarfs, and Neutron Stars (New York: Wiley, 1983) [Translated into Russian (Moscow: Mir, 1985)]

12. Begelman M C, Blandford R D, Rees M J Rev. Mod. Phys. 56255 (1984) [Translated into Russian: in Fizika Vnegalakticheskikh Istochnikov Radioizlucheniya (Ed. R D Dagkesamanskii) (Moscow: Mir, 1987) p. 1

13. Rees M J Annu. Rev. Astron. Astrophys. 22471 (1984) 14. Zeldovich Ya B, Novikov I D Relyativistskaya Astrofizika (Relativistic Astrophysics) (Moscow: Nauka, 1967) [Translated into English (Chicago: Univ. of Chicago Press, 1971,1983)]

15. Lynden-Bell D Nature 223690 (1969)

16. Bisnovatyi-Kogan G S, Ruzmaikin A A Astrophys. Space Sci. 2845 (1974)

17. Bisnovatyi-Kogan G S, Ruzmaikin A A Astrophys. Space Sci. 42 401(1976)

18. Novikov I D, Frolov V P Fizika Chernykh Dyr (Physics of Black Holes) (Moscow: Nauka, 1986) [Translated into English (Dordrecht: Kluwer Acad., 1989)]

19. Junor W, Biretta J A, Livio M Nature 401891 (1999)

20. Perley R A, Dreher J W, Cowan J J Astrophys. J. 285 L35 (1984)

21. Lobanov A, Hardee P, Eilek J New Astron. Rev. 47629 (2003)

22. Kovalev Y Y et al. Astrophys. J. 668 L27 (2007)

23. Junor W, Biretta J A Astron. J. 109500 (1995)

24. Reynolds C S et al. Astrophys. J. 283873 (1996)

25. Sikora M, Madejski G Astrophys. J. 534109 (2000)

26. Blandford R D, Rees M J Astrophys. J. 169395 (1974)

27. Fabian A C, Rees M J Astrophys. J. 277 L55 (1995)

28. Feretti L et al. Astron. Astrophys. 298699 (1995)

29. Cheng A Y S, O'Dell S L Astrophys. J. 251 L49 (1981)

30. Proga D, Stone J M, Kallman T R Astrophys. J. 543 $686(2000)$

31. Ghisellini G et al. Astrophys. J. 362 L1 (1990)

32. Konigl A, Kartje J F Astrophys. J. 434446 (1994)

33. Blandford R D, Znajek R L Astrophys. J. 179433 (1977)

34. Beskin V S, Istomin Y N, Parev V I Astron. Zh. 691258 (1992) [Sov. Astron. 36642 (1992)]

35. Hirotani K, Okamoto I Astrophys. J. 497563 (1998)

36. Fender R P, in Compact Stellar X-ray Sources (Eds W Lewin, M van der Klis) (Cambridge: Cambridge Univ. Press, 2006) p. 381

37. Spencer R E Nature 282483 (1979)
38. Mirabel I F, Rodriguez L F Nature 37146 (1994)

39. Akerlof C et al. Nature 398400 (1999)

40. Postnov K A Usp. Fiz. Nauk 169545 (1999) [Phys. Usp. 42469 (1999)]

41. Ruderman M Ann. New York Acad. Sci. 262164 (1975)

42. Panaitescu A, Kumar P Astrophys. J. 571779 (2002)

43. Blinnikov S I et al. Pis'ma Astron. Zh. 10422 (1984)

[Sov. Astron. Lett. 10177 (1984)]

44. Eichler D et al. Nature 340126 (1989)

45. Paczynski B Acta Astron. 41257 (1991)

46. Woosley S E Astrophys. J. 405273 (1993)

47. Paczynski B Astrophys. J. 494 L45 (1998)

48. Meszaros P, Rees M J Astrophys. J. 482 L29 (1997)

49. van Putten M H P M, Levinson A Astrophys. J. $\mathbf{5 8 4} 937$ (2003)

50. Komissarov S S, Barkov M V Astrophys. J. 3821029 (2007)

51. Usov V V Nature 357472 (1992)

52. Thompson C, Duncan R C Astrophys. J. 275255 (1995)

53. Hewish A et al. Nature 217708 (1968)

54. Baade W, Zwicky F Proc. Natl. Acad. Sci. 20254 (1934)

55. Landau L D, Lifshitz E M Teoriya Polya (The Classical Theory of Fields) (Moscow: Nauka, 1973) [Translated into English (Oxford: Pergamon Press, 1975)]

56. Beskin V S, Gurevich A V, Istomin Ya N Physics of the Pulsar Magnetosphere (Cambridge: Cambridge Univ. Press, 1993)

57. Mestel L, Panagi P, Shibata S Astrophys. J. 309388 (1999)

58. Weisskopf M C et al. Astrophys. J. 536 L81 (2000)

59. Helfand D J, Gotthelf E V, Halpern J P Astrophys. J. 556380 (2001)

60. Chiueh T, Li Z-Y, Begelman M C Astrophys. J. 505835 (1998)

61. Lyubarsky Y, Kirk J G Astrophys. J. 547437 (2001)

62. Petri J, Lyubarsky Y Astron. Astrophys. 473683 (2007)

63. Kennel C F, Coroniti F V Astrophys. J. 283694 (1984)

64. Komissarov S S, Lyubarsky Y E Astrophys. J. 349779 (2004)

65. Bogovalov S V et al. Astrophys. J. 358705 (2005)

66. Del Zanna L, Amato E, Bucciantini N Astron. Astrophys. 4211063 (2004)

67. Djorgovsky S, Evans C R Astrophys. J. Lett. 335 L61 (1988)

68. Herbig G H Astrophys. J. 11111 (1950)

69. Haro G Astron. J. 5572 (1950)

70. Reipurth B, Bally J Annu. Rev. Astron. Astrophys. 39 403 (2001)

71. Surdin V G Rozhdenie Zvezd (The Birth of Stars) (Moscow: URSS, 2001)

72. Bacciotti F et al. Astrophys. J. 576222 (2002)

73. Coffey D et al. Astrophys. J. 663350 (2007)

74. Chrysostomou A, Lucas P W, Hough J H Nature 45071 (2007)

75. Pelletier G, Pudritz R E Astrophys. J. 394117 (1992)

76. Edwards S Proc. Int. Astron. Union 3171 (2007)

77. Shu F H et al. Astrophys. J. 429797 (1994)

78. Pudritz R E, Norman C A Astrophys. J. 301571 (1986)

79. Brenneman L W, Reynolds C S Astrophys. J. 6521028 (2006)

80. Daly R A Astrophys. J. Lett. 696 L32 (2009)

81. Lyne A G, Graham-Smith F Pulsar Astronomy 2nd ed. (Cambridge: Cambridge Univ. Press, 1998) 
82. Bisnovatyi-Kogan G S, Lovelace R V E New Astron. Rev. 45663 (2001)

83. Alfven H, Falthammar C-G Cosmical Electrodynamics (Oxford: Clarendon Press, 1963) [Translated into Russian (Moscow: Mir, 1967)]

84. Weber E J, Davis L (Jr.) Astrophys. J. 148217 (1967)

85. Solov'ev L S, in Voprosy Teorii Plazmy (Reviews of Plasma Physics) Vol. 3 (Ed. M A Leontovich) (Moscow: Gosatomizdat, 1963) p. 245 [Translated into English (New York: Consultants Bureau, 1967) p. 277

86. Heinemann M, Olbert S J Geophys. Res. 832457 (1978)

87. Okamoto I Astrophys. J. 173357 (1975)

88. Heyvaerts J, in Plasma Astrophysics (Lecture Notes Phys., Vol. 468, Eds C Chiuderi, G Einaudi) (Berlin: Springer-Verlag, 1996) p. 31

89. Shafranov V D Zh. Eksp. Tear. Fiz. 33710 (1957) [Sov. Phys. JETP 6545 (1958)]

90. Grad H Rev. Mod. Phys. 32830 (1960)

91. Von Mises R Mathematical Theory of Compressible Fluid Flow (New York: Academic Press, 1958) [Translated into Russian (Moscow: IL, 1961)]

92. Guderley K G Theorie Schallnaher Strbmungen (Theory of Transonic Flow) (Berlin: Springer-Verlag, 1957) [Translated into English (Oxford: Pergamon Press, 1962); Translated into Russian (Moscow: IL, I960)]

93. Frankl F I Izbrannye Trudy po Gazovoi Dinamike (Selected Papers on Gasdynamics) (Moscow: Nauka, 1973)

94. Ardavan H Astrophys. J. 204889 (1976)

95. Lovelace R V E et al. Astrophys. J. Suppl. 621 (1986) 96. Nitta S, Takahashi M, Tomimatsu A Phys. Rev. D 44 2295 (1991)

97. Beskin V S, Par'ev V I Usp. Fiz. Nauk 16395 (1993) [Phys. Usp. 36529 (1993)]

98. Contopoulos I, Kazanas D, Fendt C Astrophys. J. 511 351 (1999)

99. Bogovalov S V Astron. Astrophys. 3711155 (2001)

100. Goodwin S P et al. Astrophys. J. 349213 (2004)

101. Gruzinov A Phys. Rev. Lett. 94021101 (2005)

102. Komissarov S S Astrophys. J. 36719 (2006)

103. McKinney J C Astrophys. J. 368 L30 (2006)

104. Timokhin A N Astrophys. J. 3681055 (2006)

105. Svensson R Astrophys. J. 209175 (1984)

106. Zakamska N L, Begelman M C, Blandford R D Astrophys. J. 679990 (2008)

107. Beskin V S, Gurevich A V, Istomin Ya N Zh. Eksp. Teor. Fiz. 85401 (1983) [Sov. Phys. JETP 58235 (1983)]

108. Beskin V S, Rafikov R R Astrophys. J. 313433 (2000)

109. Tchekhovskoy A, McKinney J C, Narayan R Astrophys. J. 388551 (2008)

110. Bogovalov S V Pis'ma Astron. Zh. 18832 (1992) [Sov. Astron. Lett. 18337 (1992)]

111. Beskin V S, Okamoto I Astrophys. J. 313445 (2000)

112. Toropina O D et al. Mem. Soc. Astron. Ital. 76508 (2005)

113. Beskin V S, Kuznetsova I V Nuovo Cimento B 115795 (2000)

114. Kazhdan Y M, Murzina M Astrophys. J. 270351 (1994)

115. Uchida Y, Shibata K Astron. Soc. Jpn. 36105 (1984)

116. Hawley J F, Smarr L L, Wilson J R Astrophys. J. 277 296 (1984)

117. Shima E et al. Astrophys. J. 217367 (1985)

118. Petrich L I et al. Astrophys. J. 336313 (1989)

119. Ustyugova G V et al. Astrophys. J. 439 L39 (1995)

120. Bogovalov S, Tsinganos K Astrophys. J. 305211 (1999)

121. Ustyugova G V et al. Astrophys. J. 541 L21 (2000)
122. Komissarov S S Astrophys. J. 326 L41 (2001)

123. Komissarov S S et al. Astrophys. J. 38051 (2007)

124. Barkov M V, Komissarov S S Astrophys. J. 385 L28 (2008)

125. Tchekhovskoy A, McKinney J C, Narayan R Astrophys. J. 6991789 (2009)

126. Komissarov S S et al. Astrophys. J. 3941182 (2009)

127. Tchekhovskoy A, Narayan R, McKinney J C Astrophys. J. 71150 (2010)

128. Lynden-Bell D Astrophys. J. 279389 (1996)

129. Lynden-Bell D Astrophys. J. 3411360 (2003)

130. Landau L D, Lifshitz E M Elektrodinamika Sploshnykh Sred (Electrodynamics of Continuous Media) (Moscow: Nauka, 1982) [Translated into English (Oxford: Pergamon Press, 1984)]

131. Thorne K S, Price R H, Macdonald D A Black Holes: the Membrane Paradigm (New Haven: Yale Univ. Press, 1986) [Translated into Russian (Moscow: Mir, 1988)]

132. Punsly B Black Hole Gravitohydromagnetics (Berlin: Springer, 2001)

133. Al'pert Ya L, Gurevich A V, Pitaevskii L P Iskusstvennye Sputniki v Razrezhennoi Plazme (Space Physics with Artifical Satellites) (Moscow: Nauka, 1964) [Translated into English (New York: Consultants Bureau, 1965)]

134. Komissarov S S Astrophys. J. 3501431 (2004)

135. Camenzind M Compact Objects in Astrophysics (Heidelberg: Springer, 2007)

136. Okamoto I Astrophys. J. 307253 (1999)

137. Heyvaerts J, Norman C Astrophys. J. 5961240 (2003)

138. Heyvaerts J, Norman C Astrophys. J. 3471055 (1989)

139. Bogovalov S V Pis'ma Astron. Zh. 21633 (1995) [Astron. Lett. 21565 (1995)]

140. Bogovalov S V Pis'ma Astron. Zh. 24381 (1998) [Astron. Lett. 24321 (1998)]

141. Sakurai T Astron. Astrophys. 152121 (1985)

142. Michel F C Astrophys. J. 180 L133 (1973)

143. Beskin V S, Kuznetsova IV, Rafikov R R Astrophys. J. 299341 (1998)

144. Tomimatsu A Publ. Astron. Soc. Jpn. 46123 (1994)

145. Beskin V S, Nokhrina E E Astrophys. J. 367375 (2006)

146. Beskin V S, Nokhrina E E Astrophys. J. 3971486 (2009)

147. Trubnikov B A Teoriya Plazmy (Plasma Theory) (Moscow: Energoatomizdat, 1996)

148. Bogovalov S V Astrophys. J. 28039 (1996)

149. Bogovalov S V Pis'ma Astron. Zh. 16844 (1990) [Sov. Astron. Lett. 16362 (1990)]

150. Lyubarsky Yu Astrophys. J. 6981570 (2009)

151. Beskin V S, Malyshkin L M Pis'ma Astron. Zh. 26253 (2000) [Astron. Lett. 26208 (2000)]

152. Kadomtsev B B Kollektivnye Yavleniya v Plazme (Cooperative Effects in Plasmas) 2nd ed. (Moscow: Nauka, 1988) [Translateed into English, in Reviews of Plasma Physics Vol. 22 (Ed. V D Shafranov) (New York: Kluwer Acad./Plenum Publ., 2001) p. 1

153. Ryutov D D, Derzon M S, Matzen M K Rev. Mod. Phys. 72167 (2000)

154. Bisnovatyi-Kogan G S, Komberg B V, Fridman A M Astron. Zh. 46465 (1969) [Sov. Astron. 13369 (1969)]

155. Benford G Astrophys. J. 247792 (1981)

156. Hardee P E Astrophys. J. 313607 (1987)

157. Hardee P E, Norman M L Astrophys. J. 33470 (1988) 158. Appl S, Camenzind M Astron. Astrophys. 256354 (1992)

159. Lyubarskii Yu E Astrophys. J. 3081006 (1999) 
160. Bisnovatyi-Kogan G Astrophys. Space Sci. 311287 (2007)

161. Ciardi A et al. Astrophys. J. 691 L147 (2009)

162. Bellan P M et al. Phys. Plasmas 16041005 (2009)

163. Istomin Y N, Pariev VI Astrophys. J. 267629 (1994)

164. Meliani Z, Keppens R Astron. Astrophys. 475785 (2007)

165. Narayan R, Li J, Tchekhovskoy A Astrophys. J. 697 $1681(2009)$

166. McKinney J C, Blandford R D Astrophys. J. 394 L126 (2009)

167. Beskin V S, Zakamska N L, Sol H Astrophys. J. 347 587 (2004)

168. Narayan R, McKinney J C, Farmer A J Astrophys. J. 375548 (2006)

169. Barkov M V, Komissarov S S Int. J. Mod. Phys. D 17 1669 (2008)

170. Bardou A, Heyvaerts J Astron. Astrophys. 3071009 (1996)

171. Lovelace R V E, Romanova M M Astrophys. J. 596 L159 (2003)

172. Michel F C Astrophys. J. 180207 (1973)

173. Mestel L, Wang Y-M Astrophys. J. 188799 (1979)

174. Lyubarskii Y E Pis'ma Astron. Zh. 1634 (1990) [Sov. Astron. Lett. 1616 (1990)]

175. Lovelace R V E, Turner L, Romanova M M Astrophys. J. 6521494 (2006)

176. Bogovalov S V Astron. Astrophys. 3491017 (1999)

177. Spitkovsky A Astrophys. J. 648 L51 (2006)

178. Ruderman M A, Sutherland P G Astrophys. J. 19651 (1975)

179. Arons J Astrophys. J. 2481099 (1981)

180. Gurevich A V, Istomin Ya N Zh. Eksp. Teor. Fiz. 89 3 (1985) [Sov. Phys.JETP 62 1(1985)]

181. Bai X-N, Spitkovsky A Astrophys. J. 7151282 (2010)

182. Kramer M et al. Science 312549 (2006)

183. Beskin V S, Nokhrina E E Astrophys. Space Sci. $\mathbf{3 0 8}$ 569 (2007)

184. Gurevich A V, Istomin Ya N Astrophys. J. 3771663 (2007)

185. Kato Y, Mineshige S, Shibata K Astrophys. J. 605307 (2004)

186. Sherwin B D, Lynden-Bell D Astrophys. J. 378409 (2007)

187. Uzdensky D A, MacFadyen A I Astrophys. J. 669546 (2007)

188. Romanova M M et al. Astrophys. J. 3991802 (2009)

189. Lovelace R V E et al. Astrophys. J. 572445 (2002)

190. Ghosh P, Abramowicz M A Astrophys. J. 292887 (1997)

191. Livio M, Ogilvie G I, Pringle J E Astrophys. J. 512100 (1999)

192. Konigl A Astrophys. J. 342208 (1989)

193. Ferreira J, Pelletier G Astron. Astrophys. 295807 (1995)

194. Krolik J H Active Galactic Nuclei, from the Central Black Hole to the Galactic Environment (Princeton, NJ: Princeton Univ. Press, 1999)

195. Derishev E V et al. Astrophys. Space Sci. 29721 (2005)

196. Stern B E, Poutanen J Astrophys. J. 3831695 (2008)

197. Sol H, Pelletier G, Asseo E Astrophys. J. 237411 (1989)

198. Cheng K S, Ho C, Ruderman M Astrophys. J. 300500 (1986)

199. Zavala R T, Taylor G B Astrophys. J. 612749 (2004)
200. Lister M L, Homan D C Astron. J. 1301389 (2005)

201. Gabuzda D C et al. Astrophys. J. 3841003 (2008)

202. Blandford R D, Konigl A Astrophys. J. 23234 (1979)

203. Lobanov A P Astron. Astrophys. 33079 (1998)

204. Churazov E M et al. Usp. Fiz. Nauk 176334 (2006) [Phys. Usp. 49319 (2006)]

205. MacFadyen A I, Woosley S E Astrophys. J. 524262 (1999)

206. Piran T Rev. Mod. Phys. 761143 (2004)

207. Tchekhovskoy A, Narayan R, McKinney J C New Astron. 15749 (2010)

208. Komissarov S S, Vlahakis N, Konigl A Astrophys. J. 40717 (2010)

209. Daugherty J K, Harding A K Astrophys. J. 252337 (1982)

210. Medin Z, Lai D Astrophys. J. 4061379 (2010)

211. Anderson J M et al. Astrophys. J. 590 L107 (2003)

212. Ferreira J, Dougados K, Cabrit S Astron. Astrophys. 453785 (2006) 